\title{
Universal RG flows across dimensions and holography
}

\author{
Nikolay Bobev ${ }^{a}$ and P. Marcos Crichigno ${ }^{b}$ \\ ${ }^{a}$ Instituut voor Theoretische Fysica, KU Leuven, \\ Celestijnenlaan 200D, B-3001 Leuven, Belgium \\ ${ }^{b}$ Institute for Theoretical Physics, University of Amsterdam, \\ Science Park 904, Postbus 94485, 1090 GL, Amsterdam, The Netherlands \\ E-mail: nikolay.bobev@kuleuven.be, p.m.crichigno@uva.nl
}

ABSTRACT: We study RG flows between superconformal field theories living in different spacetime dimensions which exhibit universal properties, independent of the details of the UV and IR theories. In particular, when the UV and IR theories are both even-dimensional we establish exact universal relations between their conformal anomaly coefficients. We also provide strong evidence for similar relations between appropriately defined free energies for RG flows between odd-dimensional theories in the large $N$ limit. Holographically, these RG flows across dimensions are described by asymptotically AdS black branes in a gauged supergravity theory, which we exhibit explicitly. We also discuss the uplift of these solutions to string and M-theory and comment on how the entropy of such black branes is captured by the dual field theory.

Keywords: AdS-CFT Correspondence, Anomalies in Field and String Theories, Renormalization Group, Conformal Field Theory

ARXIV EPRINT: 1708.05052 


\section{Contents}

1 Introduction and summary 1

1.1 Main results 2

2 Generalities 5

2.1 't Hooft anomalies 5

2.2 Weyl anomaly 6

2.3 Topological twist 8

3 Field theory $\quad 9$

3.1 6d SCFTs 10

$\begin{array}{ll}3.1 .1 \mathcal{N}=(1,0) & 10\end{array}$

$\begin{array}{ll}3.1 .2 \mathcal{N}=(2,0) & 13\end{array}$

$3.24 \mathrm{~d}$ SCFTs 15

$\begin{array}{lll}3.2 .1 \mathcal{N}=1 & 15\end{array}$

$\begin{array}{lll}3.2 .2 \mathcal{N}=2 & 16\end{array}$

3.2.3 Comments on $\mathcal{N}=3$ and $\mathcal{N}=4$ SCFTs 19

3.3 SCFTs in odd dimensions 20

3.4 Comments on Hofman-Maldacena bounds 22

4 Gauged supergravity $\quad 24$

$4.17 \mathrm{~d}$ supergravity 26

$\begin{array}{ll}4.1 .1 \mathcal{N}=2 & 26\end{array}$

$\begin{array}{lll}4.1 .2 \mathcal{N}=4 & 28\end{array}$

4.2 6d supergravity 31

4.3 5d supergravity 33

4.3.1 $\mathcal{N}=2 \quad 33$

$\begin{array}{lll}4.3 .2 \mathcal{N}=4 & 34\end{array}$

4.4 4d supergravity 37

$\begin{array}{lll}4.4 .1 \mathcal{N}=2 & 37\end{array}$

$\begin{array}{lll}4.4 .2 \mathcal{N}=4 & 38\end{array}$

4.5 Uplifts to $10 \mathrm{~d}$ and 11d 40

5 Discussion 41

A Conventions and normalizations $\quad 45$

$\begin{array}{lll}\text { A.1 Characteristic classes } & 45\end{array}$

A.2 Metric on Riemann surfaces 46

B Entropy of black branes $\quad 46$

C Universal RG flows in the same dimension $\quad 47$ 


\section{Introduction and summary}

Supersymmetric quantum field theories (QFTs) placed in background fields provide a rich laboratory for testing our understanding of quantum theories. The toolbox for studying such theories has greatly expanded in recent years, leading to a cornucopia of exact results such as the nonperturbative computation of physical observables, nontrivial tests of holography and other known dualities, and the discovery of many new dualities. In the landscape of consistent supersymmetric QFTs, superconformal field theories (SCFTs) play a distinguished role. Their enhanced symmetry offers greater calculational control and they serve as anchors around which to structure our understanding of the renormalization group (RG) flow. SCFTs in background fields will be the main objects of interest in this work.

Consider placing an SCFT on a curved manifold $M_{d}$. If the manifold is equipped with a conformally flat metric, superconformal symmetry is preserved (up to well-understood anomalies in even dimensions). On a general curved manifold, however, both supersymmetry and conformal symmetry are generically broken, which leads to a reduced computational control. This can be remedied by employing a simple and powerful idea, due to Witten [1]. Loosely speaking, the basic observation is that for supersymmetric QFTs with a continuous global R-symmetry one can turn on a background gauge field for this symmetry, and tune its magnitude so as to cancel (part) of the curvature of the manifold. This procedure, dubbed a topological twist, ensures that there is a particular covariantly constant spinor defined on the curved background, which can be used as a supersymmetry generator. ${ }^{1}$

While Witten's idea was originally used to obtain a topological QFT on $M_{d}$, here we will be interested in a different application, possible when the manifold has a product structure of the form $M_{d}=\mathbb{R}^{p} \times M_{d-p}$, where $\mathbb{R}^{p}$ is flat Euclidean space and $M_{d-p}$ is a general curved, smooth, compact, and orientable manifold. ${ }^{2}$ In this case one needs to perform a topological twist on $M_{d-p}$ only, a procedure often referred to as a partial topological twist. Then, at length scales much larger than the one set by $M_{d-p}$, the effective dynamics is controlled by a non-topological supersymmetric theory on $\mathbb{R}^{p}$. This procedure can be interpreted as an RG flow across dimensions, triggered by the local operators in the $d$-dimensional UV SCFT which are turned on by the background fields implementing the twist. This procedure is often applied in the literature to study specific QFTs, and has led to many new insights into the physics of the resulting low energy $p$-dimensional theory, as well as to unexpected dualities between this theory and a topological theory on $M_{d-p}$; see [3] for a recent review and further references.

If, in addition to a continuous R-symmetry, the $d$-dimensional SCFT in the UV has continuous flavor symmetries, one is free to turn on background values for the corresponding flavor gauge fields, without breaking any additional supersymmetry. While this freedom often leads to a large and interesting zoo of $p$-dimensional SCFTs in the IR (see [4-15] for

\footnotetext{
${ }^{1}$ There are more general ways to place a supersymmetric QFT on a curved manifold; see for example [2] for a systematic approach. In this work we focus on the topological twist.

${ }^{2}$ For concreteness, here we focus on QFTs in Euclidean signature, although the discussion is also valid for Lorentzian theories, and we later switch between both signatures. We assume that $M_{d-p}$ is smooth and compact, in particular there are no boundaries, punctures, or other defects, but it should be possible to relax this requirement.
} 
a selection of recent references), it is clear that the details of such constructions depend on the particular choice of UV SCFT and background flavor fields. In contrast, any QFT has a stress-energy tensor which, for the SCFTs of interest here, sits together with the superconformal R-symmetry current in a supercurrent multiplet. The universality of this multiplet structure suggests that twisted compactifications involving only the metric and the exact superconformal R-symmetry gauge field posses special properties, common to all $d$ dimensional UV SCFTs with a given amount of supersymmetry. Indeed, as we demonstrate below, this expectation bears out.

A main objective of this work is to establish universal properties of this class of RG flows. We shall assume that the UV and IR theories at both ends of the RG flow are interacting SCFTs. Since SCFTs do not exist in dimension greater than six, we take $d \leq 6$. Since we are interested in obtaining dynamical theories on $\mathbb{R}^{p}$, we consider $p \geq 1$, and since no topological twist is required on $S^{1}$ we take $p \leq d-2$. The tools we employ to study this setup are general and non-perturbative. Since the construction is clearly strongly dependent on the identification of the correct superconformal R-symmetry, we make use of the various maximization/extremization principles which determine the exact superconformal R-current $[6,7,16,17]$. Another powerful tool we use, when both $p$ and $d$ are even, is 't Hooft anomaly matching. As we discuss in detail below, this allows us to establish universal relations between conformal anomalies in the UV and IR theories. In the case of 3d SCFTs placed on Riemann surfaces (i.e., $d=3, p=1$ ) we use recent supersymmetric localization results to identify a universal relation between certain supersymmetric partition functions. Finally, another important weapon in our arsenal is the holographic duality. We now proceed to present a short summary of the main results of our work.

\subsection{Main results}

Consider a $d$-dimensional SCFT with a continuous R-symmetry and, possibly, global flavor symmetries. The corresponding currents organize themselves into supermultiplets of the form:

$$
\left\{T_{\mu \nu}, J_{\mu}^{R}, \cdots\right\}, \quad\left\{J_{\mu}^{F}, \cdots\right\},
$$

where $T_{\mu \nu}$ is the stress-energy tensor, $J_{\mu}^{R}$ is the exact superconformal R-symmetry current, and $J_{\mu}^{F}$ are flavor currents. The ellipsis stand for other possible bosonic and fermionic operators which depend on the number of supercharges and the dimension of spacetime. While the stress-energy tensor multiplet is omnipresent in SCFTs, the flavor symmetry currents depend on the specific theory under consideration. As described above, the topological twist is implemented by giving the corresponding sources, $\left\{g_{\mu \nu}, A_{\mu}^{R} \cdots\right\}$ and $\left\{A_{\mu}^{F}, \cdots\right\}$, nontrivial background values. This procedure triggers an RG flow which depends on the details of the UV theory and in general requires a case-by-case analysis. The basic observation we make here is that if the topological twist is implemented by turning on only background fields which couple to the stress-energy tensor multiplet $\left\{T_{\mu \nu}, J_{\mu}^{R}, \cdots\right\}$, the resulting RG flow exhibits universal properties. Based on this observation, we make the following definition: Given a d-dimensional UV SCFT with a certain number of supercharges and continuous 
$R$-symmetry, placed on $\mathbb{R}^{p} \times M_{d-p}$, we define the universal twist of the theory as the partial topological twist on $M_{d-p}$ along the exact $U V$ superconformal $R$-symmetry, preserving the maximal possible number of supercharges. We note that for theories with a large amount of supersymmetry there may be more than one universal twist and we discuss this in detail below. For theories with rational central charges one may find constraints on the topology of the compactification manifold $M_{d-p}$ due to Dirac quantization of the background gauge field (see section 2.3). ${ }^{3}$

As is clear by now, the exact superconformal R-symmetry plays a crucial role in our story. Upon a generic (non-universal) twisted compactification of the theory, the exact R-symmetry of the IR SCFT may differ from the one in the UV, due to possible mixing of Abelian R-symmetries with Abelian flavor symmetries along the flow. Although the precise mixing may be determined by an appropriate extremization principle, the result depends on the details of the theory and is thus not universal. In the case of flows between evendimensional SCFTs with a universal twist, however, one can show that there is no such mixing along the flow. ${ }^{4}$ This in turn leads to universal relations between the conformal anomaly coefficients, which we denote by the vector $(a, \vec{c})$, of the UV $d$-dimensional theory and the IR $p$-dimensional theory of the form:

$$
\left(\begin{array}{l}
a_{p} \\
\vec{c}_{p}
\end{array}\right)=\mathcal{U}\left(\begin{array}{l}
a_{d} \\
\vec{c}_{d}
\end{array}\right) .
$$

Here $\mathcal{U}$ is a matrix that depends only on the topology of the compactification manifold, $M_{d-p}$, and the data specifying the partial topological twist, but is independent of the details of the SCFTs at both ends of the RG flow. These relations are exact and do not rely on the existence of a Lagrangian description of the UV or IR SCFTs.

We will argue that this universal behavior is not limited to RG flows between evendimensional theories and that similar universal relations exist for flows between theories in various dimensions. For RG flows between odd-dimensional theories one cannot rely on 't Hooft anomalies but in view of the $F$-theorem [18] it is natural to search for universal relations between the round-sphere free energy of the UV and IR SCFTs. Although computing free energies exactly is much harder than computing anomalies, we are nonetheless able to show that the free energies indeed are related by

$$
F_{S^{p} \times M_{d-p}}=u F_{S^{d}}
$$

to leading order in $N$, in an appropriate large $N$ limit. Here $u$ is again a universal coefficient, depending only on the topology of $M_{d-p}$ and the topological twist performed, but not the details of the SCFTs. One example of such a universal flow between odd dimensions arises from considering $3 \mathrm{~d} \mathcal{N}=2$ SCFTs compactified on a Riemann surface to a quantum mechanical system. In this case (1.3) can be established by pure field theory methods,

\footnotetext{
${ }^{3}$ For theories with an Abelian R-symmetry the R-charges may be irrational, which renders the universal twist impossible. These subtleties do not arise if the UV theory has a non-Abelian R-symmetry.

${ }^{4}$ There is a minor caveat in the case of $4 \mathrm{~d} \mathcal{N}=1$ SCFTs compactified on a Riemann surface, which we discuss in section 3.2.1.
} 
by an analysis of the corresponding matrix models at large $N$ [19] (see section 3.3). For theories in other dimensions we will establish (1.3) using holography. It would be interesting to establish these relations also in field theory.

As mentioned above, we assume that the RG flow from the SCFT in the UV ends at an interacting SCFT in the IR. Whether this is actually the case is a nontrivial dynamical question, difficult to establish by field theory methods alone. When the $d$-dimensional SCFT admits a weakly coupled holographic dual, however, one can bring holography to bear on this question. Indeed, one way to establish the existence of an interacting superconformal fixed point in the IR (at least in the planar limit) is by constructing a supergravity solution that explicitly interpolates between the UV and IR SCFTs. The holographic description of twisted compactifications was first studied in the foundational work of Maldacena and Núñez (MN) [20], which built upon results for D-branes wrapping calibrated cycles [21] (see [22] for a review and further references). We exploit the same approach in our holographic analysis.

In this holographic setting, it is natural to ask what is the supergravity manifestation of universal RG flows across dimensions. Consider the supergravity fields dual to the operators in (1.1). These also organize themselves into multiplets, of the form

$$
\left\{g_{\mu \nu}, A_{\mu}^{R}, \cdots\right\}, \quad\left\{A_{\mu}^{F}, \cdots\right\},
$$

where the gravity multiplet contains the metric $g_{\mu \nu}$ and the graviphoton $A_{\mu}^{R}$. The gauge fields $A_{\mu}^{F}$ belong to vector multiplets. ${ }^{5}$ Since universal twists involve only operators dual to the gravity multiplet, it is natural to expect that the dynamics of this multiplet is sufficient to capture the corresponding universal RG flow. Indeed, one can restrict to this "minimal" gauged supergravity theory ${ }^{6}$ in $(d+1)$ dimensions and construct domain wall solutions with a metric of the form

$$
d s_{d+1}^{2}=e^{2 f(r)} d s_{\mathbb{R}^{p+1}}^{2}+e^{2 g(r)} d s_{M_{d-p}}^{2} .
$$

Here $r$ is the "holographic" direction, and the metric is locally asymptotic to $\operatorname{AdS}_{d+1}$ for large $r$ (the UV) and approaches $\mathrm{AdS}_{p+1} \times M_{d-p}$ for small $r$ (the IR). The solution is supported by a nontrivial magnetic flux for the graviphoton through 2-cycles in $M_{d-p}$. The entire spacetime can be thought of as a magnetically charged BPS $(p-1)$-brane living in $\mathrm{AdS}_{d+1}$, interpolating between the $\mathrm{SCFT}_{d}$ dual in the UV and the $\mathrm{SCFT}_{p}$ dual in the IR. We identify the black brane solutions corresponding to various twisted compactifications in minimal gauged supergravity for $(d+1)=4,5,6,7$ and describe them in detail in section 4. In addition, we show that the field theory universal relations (1.2) and (1.3) are correctly reproduced holographically. Although many of these supergravity solutions have been found in the literature before, their field theory interpretation as universal RG flows has not necessarily been appreciated.

To make contact with top-down constructions in string and M-theory we also emphasize that these low-dimensional gauged supergravity theories arise as consistent truncations

\footnotetext{
${ }^{5}$ These are dynamical supergravity fields and coincide with the background metric and gauge fields in the boundary field theory discussed below (1.1) only at the asymptotically AdS boundary.

${ }^{6}$ Note that by "minimal" here we mean that we consider only the theory containing the gravity multiplet and no extra matter multiplets, not that the theory has the minimal number of supercharges.
} 
from ten- and eleven-dimensional supergravity. This means that it is possible to uplift the universal holographic RG flows to string and M-theory. The choice of the internal manifold determines the details of the particular SCFT being compactified. For instance, uplifting a five-dimensional solution to IIB SUGRA with $S^{5}$ as internal manifold describes a twisted compactification of $\mathcal{N}=4 \mathrm{SYM}$, while taking the internal manifold to be $Y^{p, q}$ corresponds to a twisted compactification of $\mathcal{N}=1$ quiver gauge theories of the type discussed in [9].

The holographic perspective not only establishes the existence of the IR fixed point, but also suggests universal relations among quantities for flows between even-dimensional and odd-dimensional SCFTs of the form

$$
a_{p}=u_{a F} F_{S^{d}}, \quad F_{S^{p} \times M_{d-p}}=u_{F a} a_{d} .
$$

Here $F$ is a free energy, $a$ is a conformal anomaly coefficient, and $u_{a F}, u_{F a}$ are again universal coefficients, depending only on the compactification manifold $M_{d-p}$ and the topological twist performed. These are nontrivial and powerful predictions that would be interesting to establish directly in field theory, including finite $N$ corrections. Finally, we should stress that the supersymmetric black branes realizing these universal RG flows across dimensions have a non-vanishing entropy. We compute these entropies in appendix B and observe interesting relations with field theory quantities such as conformal anomalies and sphere free energies.

The idea of universal RG flows common to a large class of SCFTs has appeared before, both in a holographic [23] as well as in a purely field-theoretic context [24, 25]. In these papers, however, the UV and IR theories live in the same number of space time dimensions. Universal supergravity domain walls dual to holographic RG flows, similar in spirit to the ones studied here, were also discussed in [7-9, 26, 27].

The rest of the paper is organized as follows. In section 2 we review some background material on 't Hooft and conformal anomalies and topological twists. Section 3 is devoted to a study of twisted compactifications of field theories in various dimensions with a particular focus on universal relations among conformal anomalies. In section 4 we present the holographic dual description of these universal twisted compactifications and discuss universal relations among various quantities, such as conformal anomalies and free energies from the holographic perspective. We conclude with a short summary and a discussion of various open problems in section 5 . In the three appendices we present our conventions on characteristic classes, a short discussion on the relation between some of our results and the entropy of extremal black branes, and comment on possible two-dimensional analogs of the universal RG flow discussed in [25].

\section{Generalities}

We begin by reviewing some general background on anomalies in QFTs and basics of topologically twisted theories. Readers familiar with this material may skip to section 3 .

\section{1 't Hooft anomalies}

In even-dimensional QFTs, classical symmetries may become anomalous at the quantum level. Quantum anomalies for local symmetries are forbidden in consistent QFTs. However, 
global (or 't Hooft) anomalies are not only allowed, but are in fact robust physical observables containing exact information about the theory (see [28] for a pedagogical review). 't Hooft anomalies for continuous global symmetries are packaged efficiently in the anomaly polynomial, $I_{d+2}$, of the theory. This is a gauge-invariant $(d+2)$-form which is a polynomial in characteristic classes for the global symmetries of the theory.

We will consider two-dimensional $\mathcal{N}=(0,2)$, four-dimensional $\mathcal{N}=1$, and sixdimensional $\mathcal{N}=(1,0)$ theories, whose R-symmetry groups are $\mathrm{U}(1)_{R}, \mathrm{U}(1)_{R}$, and $\mathrm{SU}(2)_{R}$, respectively. $^{7}$ In addition, these theories generically also have flavor symmetries. The anomaly polynomials are given by:

$$
\begin{aligned}
2 \mathrm{~d}: & I_{4}=\frac{k_{R R}}{2} c_{1}\left(\mathcal{F}_{R}\right)^{2}-\frac{k}{24} p_{1}\left(\mathcal{T}_{2}\right)+I_{4}^{\text {flavor }} \\
4 \mathrm{~d}: & I_{6}=\frac{k_{R R R}}{6} c_{1}\left(\mathcal{F}_{R}\right)^{3}-\frac{k_{R}}{24} c_{1}\left(\mathcal{F}_{R}\right) p_{1}\left(\mathcal{T}_{4}\right)+I_{6}^{\text {flavor }} \\
6 \mathrm{~d}: & I_{8}=\frac{1}{4 !}\left(\alpha c_{2}^{2}\left(\mathcal{F}_{R}\right)-\beta c_{2}\left(\mathcal{F}_{R}\right) p_{1}\left(\mathcal{T}_{6}\right)+\gamma p_{1}^{2}\left(\mathcal{T}_{6}\right)+\delta p_{2}\left(\mathcal{T}_{6}\right)\right)+I_{8}^{\text {flavor }} .
\end{aligned}
$$

Here $c_{n}(\mathcal{F})$ denotes the $n$ 'th Chern class of the corresponding bundle and $p_{n}\left(\mathcal{T}_{d}\right)$ denotes the $n$ 'th Pontryagin class of the tangent bundle of the manifold on which the theory is placed. See appendix A.1 for our conventions. The various coefficients multiplying these characteristic classes encode the corresponding 't Hooft anomalies for the energy-momentum multiplet in the theory. For the theories considered here this multiplet always contains the superconformal R-symmetry current. If the QFT at hand admits a Lagrangian description these anomalies can be computed by one-loop Feynman diagrams with insertions of the R-current and the energy-momentum tensor.

In the anomaly polynomials above we have not included anomalies for gauge symmetries since in this work we only study consistent QFTs, where such anomalies are absent. Note that we allow for gravitational anomalies since we are discussing QFTs and thus the metric is treated as a non-dynamical background field. We have not given explicit expressions for any flavor anomalies, either pure or mixed with R-symmetry or gravitational anomalies. These are schematically encoded in the anomaly polynomial $I_{d+2}^{\text {flavor }}$ in $(2.1)-(2.3)$. The rationale for doing this is that these anomalies depend on the details of the theory and can be ignored (under certain mild assumptions, to be discussed in section 3) for the purposes of our discussion.

\subsection{Weyl anomaly}

Another important anomaly for our story is the Weyl (or conformal) anomaly, which captures the failure of the stress-energy tensor to be traceless when an even-dimensional CFT is placed in a nontrivial curved background. Ignoring conventional normalizations the anomaly has the form:

$$
\left\langle T_{\mu}^{\mu}\right\rangle \sim a E_{d}+\sum_{i} c_{i} W_{i}
$$

\footnotetext{
${ }^{7}$ This formalism is also applicable for even-dimensional theories with more supersymmetry and we use it extensively in this context in section 3 .
} 
where $E_{d}$ is the Euler density in $d$ dimensions and the $W_{i}$ are a set of local, independent, Weyl invariants of the manifold on which the theory is placed. The number of independent invariants of this type depends on the spacetime dimension. There are none in two dimensions, one in four dimensions, and three in six dimensions.

In a superconformal theory, the stress-energy tensor and the superconformal R-symmetry current sit in the same supermultiplet. As a consequence, the Weyl and R-symmetry anomaly coefficients in (2.1)-(2.3) and (2.4) are related by supersymmetry through Ward identities. In 2d (see for example [29]) and 4d [30] these relations are:

$$
\begin{aligned}
c_{r} & =3 k_{R R}, & c_{r}-c_{l} & =k, \\
a_{4 d} & =\frac{9}{32} k_{R R R}-\frac{3}{32} k_{R}, & c_{4 d} & =\frac{9}{32} k_{R R R}-\frac{5}{32} k_{R},
\end{aligned}
$$

where $R$ denotes the exact superconformal R-symmetry.

In $6 \mathrm{~d}$ there are three tensor structures $W_{i}$ and thus three $c$-type coefficients (see e.g. [31] and references therein for details). For theories with $\mathcal{N}=(1,0)$ supersymmetry there is a linear relation ${ }^{8}$ among the $c_{i}$ 's and thus the independent Weyl anomaly coefficients in $6 \mathrm{~d}$ can be taken to be $a_{6 d}, c_{6 d}^{(1)}, c_{6 d}^{(2)}$. The expression for the $a$-anomaly coefficient in terms of R-symmetry anomalies was found in [33]. The expression for the $c_{6 d}^{(1)}, c_{6 d}^{(2)}$ coefficients was recently determined in [34-36]. The result is given by the following formulae:

$$
\begin{aligned}
a_{6 d} & =\frac{2}{7}(8 \alpha-8 \beta+8 \gamma+3 \delta) a_{T}, \\
c_{6 d}^{(1)} & =\frac{256}{7}(6 \alpha-7 \beta+8 \gamma+4 \delta) a_{T}, \\
c_{6 d}^{(2)} & =\frac{64}{7}(6 \alpha-5 \beta+4 \gamma+5 \delta) a_{T},
\end{aligned}
$$

where $a_{T}$ is the value of $a_{6 d}$ for the free $\mathcal{N}=(2,0)$ tensor multiplet. ${ }^{9}$ In the special case of $\mathcal{N}=(2,0)$ theories one has the relations [34], $\gamma=\frac{\beta}{4}$ and $\delta=-\beta$ which imply that $c_{6 d}^{(1)}=4 c_{6 d}^{(2)}$ and there is only one independent $c$-type coefficient, which we take to be $c_{6 d} \equiv \frac{7 c_{6 d}^{(1)}}{384}=\frac{7 c_{6 d}^{(2)}}{96} .{ }^{10}$ The relations $(2.6)$ for $\mathcal{N}=(2,0)$ SCFTs then simplify to

$$
a_{6 d}=\frac{16}{7}\left(\alpha-\frac{9}{8} \beta\right), \quad c_{6 d}=4\left(\alpha-\frac{3}{2} \beta\right) .
$$

For $\mathcal{N}=(2,0)$ SCFTs in the ADE class one has

$$
\alpha=d_{G} h_{G}+r_{G}, \quad \beta=4 \gamma=-\delta=\frac{r_{G}}{2},
$$

where $d_{G}, r_{G}$, and $h_{G}$ are the dimension, rank, and Coxeter number of the group $G$, respectively, satisfying the group theory identity $d_{G}=r_{G}\left(1+h_{G}\right)$. We note that for these theories $\frac{4}{7} \leq a_{6 d} / c_{6 d} \leq 1$, the lower bound being saturated in the large $N$ limit for the $A_{N}$ and $D_{N}$ theories.

\footnotetext{
${ }^{8} \mathrm{As}$ argued in [32] the relation imposed by supersymmetry is $6 c_{6 d}^{(3)}+c_{6 d}^{(1)}-2 c_{6 d}^{(2)}=0$.

${ }^{9} \mathrm{In}$ [34] this was normalized to $a_{T}=-\frac{7}{1152}$. Here we follow the conventions in [33] and set $a_{T}=1$.

${ }^{10}$ Compared to [34] we use the normalization $c_{6 d}=\frac{7}{4} c$ so that the $c$-type coefficient for the free tensor multiplet is 1 .
} 


\subsection{Topological twist}

If a supersymmetric QFT in flat space is placed in a general background for the metric and gauge fields, supersymmetry will be broken. It was recently understood how to systematically arrange the background fields in such a way that some amount of supersymmetry is preserved [2]. Here we will specialize to one particular such way, introduced by Witten in [1] and known as a topological twist. The basic idea can be summarized as follows. If the QFT at hand has a continuous R-symmetry, one can turn a background gauge field, $A_{\mu}^{R}$, that couples to the R-symmetry current. One can then adjust the magnitude of the background field so as to cancel the nontrivial part of the spin connection $\omega_{\mu}$ on the curved manifold. Schematically, one tunes $A_{\mu}^{R}=-\frac{1}{4} \omega_{\mu}$ so that the generalized Killing spinor equation takes the form

$$
\tilde{\nabla}_{\mu} \epsilon=\left(\partial_{\mu}+\frac{1}{4} \omega_{\mu}+A_{\mu}^{R}\right) \epsilon=\partial_{\mu} \epsilon=0 .
$$

The last equation in (2.9) admits a constant spinor solution on any spin manifold, implying that some amount of supersymmetry is preserved in this nontrivial background for the metric and R-symmetry gauge field. ${ }^{11}$ It is clear that this method for preserving some supersymmetry can work only if the nontrivial part of the spin connection can be embedded in the R-symmetry group of the QFT in flat space. ${ }^{12}$

The case of interest to us here is when $M_{d}$ is a product manifold of the form $M_{d}=$ $\mathbb{R}^{p} \times M_{d-p}$, with $M_{d-p}$ a curved, compact, and smooth manifold. Then, the topological twist is performed only along $M_{d-p}$ and thus at low energies (compared to the scale set by the size of $M_{d-p}$ ) one expects to have a physical, i.e., non-topological, supersymmetric theory on $\mathbb{R}^{p}$. This is known as a partial topological twist. ${ }^{13}$ An important point for many of our constructions below is that this topological twist is naturally realized in string or M-theory on the world-volume of D- or M-branes wrapping calibrated cycles in special holonomy manifolds [21].

If, in addition to a continuous R-symmetry, the QFT at hand has a continuous flavor symmetry, one can turn a more general background $A_{\mu}^{R^{\prime}}=A_{\mu}^{R}+\sum_{i} a_{i} A_{\mu}^{F_{i}}$, where $A_{\mu}^{F_{i}}$ are background fields for the flavor symmetry and the $a_{i}$ are free parameters. Since, by definition, the supersymmetry parameter $\epsilon$ is not charged under flavor symmetries, the Killing spinor equation (2.9) is not modified by turning on background flavor fields and the amount of supersymmetry preserved is unchanged. When the R-symmetry group is

\footnotetext{
${ }^{11}$ Here we have been schematic, omitting Lorentz and R-symmetry indices. If these are included one sees that the cancellation of the spin connection by the background R-symmetry typically requires conditions on the spinor such as being chiral in the two-dimensional case. Generally speaking the cancellation can occur, at most, when acting on half of the components of $\epsilon$ and thus, at most, half supersymmetry can be preserved in this way.

${ }^{12}$ We are being slightly imprecise here. In general, one needs to cancel only part of the spin connection on the curved manifold if the spin connection after the twist admits covariantly constant spinors. For example, on Kähler manifolds in four real dimensions one may cancel only the $\mathrm{U}(1)$ part of the $\mathrm{U}(2)=\mathrm{U}(1) \times \mathrm{SU}(2)$ structure group.

${ }^{13}$ In the original construction of Witten the manifold $M_{d}$ did not necessarily have a product structure with a flat factor and thus the resulting theory on the curved manifold was topological. Although interesting, we do not consider such theories here.
} 
Abelian this freedom reflects the fact that the R-symmetry is ambiguous, as any linear combination of a "reference" R-symmetry with Abelian flavor symmetries is again an Rsymmetry. Although one is free to choose any reference R-symmetry one likes, in the case of SCFTs there is a preferred R-symmetry, namely the superconformal R-symmetry, $R_{S C}$, whose corresponding current belongs to the stress-energy tensor supermultiplet. This unique R-symmetry can be determined by maximization/extremization principles in any integer dimension in the range $1 \leq d \leq 4[6,7,12,16,17]$. For SCFTs it is thus natural to take the superconformal R-symmetry to be the reference R-symmetry and write the background field as:

$$
A_{\mu}^{R}=A_{\mu}^{R_{\mathrm{SC}}}+\sum_{i} a_{i} A_{\mu}^{F_{i}}
$$

It is then easy to understand why the universal RG flows across dimensions studied in this paper are special; these correspond to setting $a_{i}=0$, i.e., the special choice of background gauge field which extends only along the exact superconformal R-symmetry in the UV. Although not the focus here, flows for generic values of $a_{i}$ are of course also interesting, and lead to a plethora of RG flows across dimensions; see for example [4-9, 12, 37].

There is an important subtlety to keep in mind when performing topological twists. For any gauge-invariant operator $\mathcal{O}$ in the theory one must impose the Dirac quantization condition

$$
\frac{1}{2 \pi} \operatorname{Tr} \int_{C_{2}} F_{R} \cdot \mathcal{O}=n \mathcal{O}, \quad n \in \mathbb{Z},
$$

where $F_{R}$ is the background R-symmetry curvature for the background field (2.10) and $C_{2}$ is any compact 2-cycle in $M_{d}$. As a consequence, for the universal topological twist to be well defined, the exact superconformal R-charge of all gauge-invariant operators in the theory must be a rational number. This may not be the case in some theories with four Poincaré supercharges, such as $3 \mathrm{~d} \mathcal{N}=2$ and $4 \mathrm{~d} \mathcal{N}=1$ SCFTs, in which case the universal topological twist is ill-defined. Nonetheless, one can easily find an infinite number of such SCFTs with rational R-charges, so this is not an important obstruction to discuss universal properties of these constructions. For theories with rational R-charges the quantization condition in (2.11) may lead to constraints on the topology of $M_{d-p}$ and we shall discuss such cases below (see table 1 and the discussion above it). As emphasized in [9], in such situations one can circumvent these constraints on the universal topological twist by including flavor magnetic fluxes $a_{i}$, and adjusting them in a way consistent with (2.11). This procedure, however, is theory-specific and thus not universal. In this paper we restrict ourselves to SCFTs, and choices of manifolds $M_{d-p}$, for which the quantization condition (2.11) is satisfied when all flavor fluxes are set to zero. This excludes, in particular, theories with irrational R-charges.

\section{$3 \quad$ Field theory}

As outlined in the Introduction, the main characters in our story are superconformal field theories with a continuous R-symmetry, which we place on a manifold of the form $M_{d}=$ $\mathbb{R}^{p} \times M_{d-p}$ with a partial topological twist on $M_{d-p}$. In this section, we consider SCFTs with 
different number of supercharges and various values of $d$ and $p$. Our goal is to extract some physical information of the low-energy effective theory on $\mathbb{R}^{p}$ at the end of the RG flow.

For flows between even-dimensional SCFTs (both $d$ and $p$ even) the basic tools that allow us to establish the universal relations (1.2) are anomaly matching and superconformal symmetry. The calculation proceeds along the lines of the analysis in [4-9, 38, 39]. Since this will be used repeatedly throughout this section, let us summarize the general strategy before studying different cases. We start with a $\mathrm{SCFT}_{d}$ with an anomaly polynomial $I_{d+2}$. Performing a partial topological twist on $M_{d-p}$ modifies the global symmetry bundles, leading to a new anomaly polynomial $I_{d+2}^{\text {twisted }}$, which we then integrate over $M_{d-p}$ to arrive at the $I_{p+2}$ anomaly polynomial of the $p$-dimensional IR theory

$$
I_{p+2}=\int_{M_{d-p}} I_{d+2}^{\mathrm{twisted}} .
$$

This equation determines the R-symmetry anomalies in the IR in terms of those in the UV, encoding the 't Hooft anomaly matching condition [40]. Assuming the UV and IR theories are superconformal we can then use superconformal Ward identities at the two fixed points to express the R-symmetry anomalies in terms of conformal anomalies. This ultimately leads to the universal relations of the form (1.2) among IR and UV central charges.

For situations in which either $d$ or $p$ is odd, one cannot rely on anomaly matching, making it harder to analyze the resulting RG flow. As argued in the Introduction, a natural quantity to consider in the absence of anomalies is an appropriate supersymmetric partition function, or free energy, of the CFT. ${ }^{14}$ This is a much harder task, not only because the computation of such partition functions is technically more involved, but also because it is not obvious how to approach such calculations in a universal way, i.e., without referring to a specific theory. Some progress on this hard question has been made recently for twisted compactifications of $3 \mathrm{~d} \mathcal{N}=2$ theories on a Riemann surface $\Sigma_{\mathfrak{g}}(d=3, p=1)$ in the planar limit. In this case one indeed finds a universal relation between the supersymmetric threesphere free energy, $F_{S^{3}}$, of the UV $3 \mathrm{~d}$ SCFT and a certain topologically twisted partition function, $F_{\Sigma_{\mathfrak{g}} \times S^{1}}$, which is identified with a Witten index of the effective 1d theory in the IR [19] (see also [42]). This relation has been established explicitly for a large class of quiver gauge theories to leading order in $N$. We discuss this in more detail in section 3.3.

\section{$3.16 \mathrm{~d}$ SCFTs}

We begin our exploration of $\mathrm{RG}$ flows across dimensions from $d=6$, the maximal dimension in which an SCFT can exist [43]. We will consider theories with both $\mathcal{N}=(1,0)$ and $\mathcal{N}=(2,0)$ supersymmetry.

\subsection{1 $\mathcal{N}=(1,0)$}

SCFTs with $\mathcal{N}=(1,0)$ supersymmetry have eight real supercharges ${ }^{15}$ and an $\mathrm{SU}(2)_{R}$ R-symmetry. They may also have global flavor symmetries. The study of these theories

\footnotetext{
${ }^{14}$ See [41] for a somewhat related proposal on how to interpolate between CFTs in even and odd dimensions.

${ }^{15}$ Here we count only real components of Poincare supercharges. When we have a conformal theory there is as usual the accompanying superconformal supercharges.
} 
has recently attracted much attention and a general formula for their anomaly polynomial was derived in [44, 45]. The relation between 't Hooft and Weyl anomalies imposed by superconformal Ward identities was found in [33-36]. We now study these theories on Riemann surfaces and on Kähler four-manifolds with a partial topological twist.

On Riemann surfaces. Consider a smooth Riemann surface $\Sigma_{\mathfrak{g}}$ with holonomy group $\mathrm{U}(1)_{\Sigma}$. There is a unique way to embed $\mathrm{U}(1)_{\Sigma}$ into $\mathrm{SU}(2)_{R}$ while preserving minimal $4 \mathrm{~d}$ $\mathcal{N}=1$ supersymmetry. At the level of line bundles, the topological twist amounts to the replacement:

$$
\mathcal{F}_{R}^{(6 d)} \rightarrow \mathcal{F}_{R}^{(4 d)}-\frac{\kappa}{2} t_{\mathfrak{g}}
$$

where $t_{\mathfrak{g}}$ is the Chern class of the tangent bundle to $\Sigma_{\mathfrak{g}}$, normalized as in (A.7), and the coefficient $-\kappa / 2$ is fixed by supersymmetry, where $\kappa$ is the normalized curvature of $\Sigma_{\mathfrak{g}}$ defined in (A.6). In this example, the general procedure outlined above is as follows. We implement the twist in the anomaly polynomial (2.3) by the replacement (3.2) and then integrate over $\Sigma_{\mathfrak{g}}$ to obtain:

$$
\int_{\Sigma_{\mathfrak{g}}} I_{8}^{\text {twisted }}=\frac{1}{12}(\mathfrak{g}-1)\left(2 \alpha c_{1}\left(\mathcal{F}_{R}\right)^{3}-\beta c_{1}\left(\mathcal{F}_{R}\right) p_{1}(T)\right)+I_{6}^{\text {flavor }},
$$

where we have used (A.7). Comparing this to (2.2) we read off the resulting 4d 't Hooft anomaly coefficients

$$
k_{R R R}=(\mathfrak{g}-1) \alpha, \quad k_{R}=2(\mathfrak{g}-1) \beta .
$$

Many $\mathcal{N}=(1,0)$ SCFTs have non-Abelian flavor symmetry groups and thus we will assume that the $\mathrm{U}(1)_{R}$ superconformal R-symmetry of the IR $4 \mathrm{~d}$ theory is the same as the Cartan subgroup of the UV SU $(2)_{R}$ preserved by the topological twist. ${ }^{16}$ With this identification, we can use (2.5) to find the following expression for the $4 \mathrm{~d}$ Weyl anomalies:

$$
\text { 4d } \mathcal{N}=1: \quad\left(\begin{array}{c}
a_{4 d} \\
c_{4 d}
\end{array}\right)=\frac{(\mathfrak{g}-1)}{32}\left(\begin{array}{cc}
9 & -6 \\
9 & -10
\end{array}\right)\left(\begin{array}{l}
\alpha \\
\beta
\end{array}\right) .
$$

Note that the $4 \mathrm{~d}$ 't Hooft and Weyl anomalies depend only on $\alpha, \beta$ and not on the purely gravitational anomalies $\gamma, \delta$. Recall that the $6 \mathrm{~d}$ Weyl anomaly coefficients are given in terms of 't Hooft anomalies in (2.6). Since there are only three independent Weyl anomaly coefficients in $\mathcal{N}=(1,0)$ theories, but four R-symmetry anomalies, it is not possible to invert the relations (2.6) to write (3.5) as a relation purely among $6 \mathrm{~d}$ and $4 \mathrm{~d}$ Weyl anomalies, as in (1.2). It is possible to do so, however, in the case of $\mathcal{N}=(2,0)$ theories, which we discuss below. We note that requiring that the ratio $a_{4 d} / c_{4 d}$ satisfies the HofmanMaldacena bound $\frac{1}{2} \leq a_{4 d} / c_{4 d} \leq \frac{3}{2}$ imposes conditions on the values of $\alpha, \beta$. We discuss Hofman-Maldacena bounds in more detail in section 3.4.

If the SCFT at hand admits a suitable large $N$ limit the pure R-symmetry anomaly dominates over gravitational anomalies, i.e., $\alpha \gg(\beta, \gamma, \delta)$ in $(2.6)$ in which case the relation (3.5) becomes

$$
c_{4 d} \simeq a_{4 d} \simeq(\mathfrak{g}-1) \frac{63}{512} a_{6 d} .
$$

\footnotetext{
${ }^{16}$ It would be nice to put this statement on a firmer footing by analyzing the general $6 \mathrm{~d} \mathcal{N}=(1,0)$ anomaly polynomials of $[44,45]$.
} 
Assuming $a_{6 d}>0$, a positive central charge in $4 \mathrm{~d}$ is obtained only for $\mathfrak{g}>1$. We will derive this universal relation from holography in section 4.1.1.

Finally, we note that the universal relation in (3.5) is satisfied for the particular models of twisted compactifications of six-dimensional $(1,0)$ SCFTs discussed in section 7 of [46]; see in particular equations (7.1) and (7.9) there.

On Kähler four-manifolds. Consider a Kähler four-manifold $M_{4}$, whose holonomy group is (contained into) $\mathrm{U}(2)_{s}=\mathrm{SU}(2)_{s} \times \mathrm{U}(1)_{s}$. We denote the first Pontryagin number and the Euler number of $M_{4}$ by $P_{1}$ and $\chi$, respectively. These can be written in terms of the Chern roots $t_{1,2}$ of the tangent bundle to $M_{4}$ as

$$
P_{1}=\int_{M_{4}}\left(t_{1}^{2}+t_{2}^{2}\right), \quad \chi=\int_{M_{4}} t_{1} t_{2} .
$$

To preserve $2 \mathrm{~d} \mathcal{N}=(0,2)$ supersymmetry we turn on a background for the Cartan of the 6d SU(2) R-symmetry, proportional to the $\mathrm{U}(1)_{s}$ spin connection. ${ }^{17}$ This amounts to

$$
\mathcal{F}_{R}^{(6 d)} \rightarrow \mathcal{F}_{R}^{(2 d)}+\frac{1}{2} t_{1}+\frac{1}{2} t_{2} .
$$

Making this replacement in the anomaly polynomial (2.3), using the relations in (A.4), and integrating the twisted anomaly polynomial over $M_{4}$ leads to the $2 \mathrm{~d}$ 't Hooft anomalies

$$
k_{R R}=\frac{1}{8} \alpha\left(P_{1}+2 \chi\right)-\frac{1}{12} \beta P_{1}, \quad k=\frac{1}{4} \beta\left(P_{1}+2 \chi\right)-(2 \gamma+\delta) P_{1} .
$$

Thus, the central charges at the $2 \mathrm{~d}$ fixed point are given by

$$
\begin{aligned}
c_{r} & =\frac{3}{8} \alpha\left(P_{1}+2 \chi\right)-\frac{1}{4} \beta P_{1}, \\
c_{l} & =\frac{1}{4}\left(\frac{3}{2} \alpha-\beta\right)\left(P_{1}+2 \chi\right)-\frac{1}{4}(\beta-8 \gamma-4 \delta) P_{1} .
\end{aligned}
$$

The same result for the two-dimensional conformal anomalies was derived recently in [48] (see in particular equations (2.36)-(2.37) there). As we shall see in section 4.1.1, the holographic dual of this flow across dimensions exists only when the Kähler manifold is negatively curved. In this case one has the following relation between the topological invariants and the volume of the manifold: ${ }^{18}$

$$
P_{1}+2 \chi=\frac{1}{2 \pi^{2}} \operatorname{vol}\left(M_{4}\right) .
$$

Using this, together with the fact noted above that $\alpha \gg(\beta, \gamma, \delta)$ in the holographic limit, (3.10) becomes

$$
c_{r} \simeq c_{l} \simeq \frac{21}{256 \pi^{2}} \operatorname{vol}\left(M_{4}\right) a_{6 d},
$$

to leading order in $N$. We will derive this universal relation holographically in section 4.1.1.

\footnotetext{
${ }^{17}$ One may also consider turning on a background gauge field proportional to the $\mathrm{SU}(2)_{s}$ spin connection. For $6 \mathrm{~d}$ theories with only $(1,0)$ supersymmetry this results in a $2 \mathrm{~d}$ theory with $(0,1)$ supersymmetry and thus no continuous R-symmetry. Our anomaly matching procedure is thus not applicable and we do not consider this case further here. For $\mathcal{N}=(2,0)$ SCFTs this twist leads to a 2 d theory with $(0,2)$ supersymmetry and was studied in section 5.1 of [47] as well as section 6.3 of [7].

${ }^{18}$ See appendix $\mathrm{H}$ of [7] for a short summary on some relevant facts on four-manifolds.
} 
The twisted compactification of a $6 \mathrm{~d} \mathcal{N}=(1,0)$ theory on a three-manifold is possible by mixing the $\mathrm{SO}(3) \simeq \mathrm{SU}(2)$ holonomy group with the $\mathrm{SU}(2)_{R}$ R-symmetry. However, in this case one does not expect to obtain a theory with a continuous R-symmetry in the IR and we do not study this case here. For $\mathcal{N}=(2,0) \mathrm{SCFT}$, however, one is equipped with an $\mathrm{SO}(5) \mathrm{R}$-symmetry group which allows for more general topological twists. We study this next.

\subsection{2 $\mathcal{N}=(2,0)$}

General twisted compactifications of $\mathcal{N}=(2,0)$ SCFTs on Riemann surfaces and on fourmanifolds were studied in [4, 5] and [7], respectively. Particular twists on Riemann surfaces, which we identify here as universal twists, were studied in [20,39]. Here we reproduce the results in these references, emphasizing the universal aspects.

We will focus on two types of topological twists involving only an Abelian background gauge field. The first is the universal twist of the $\mathcal{N}=(1,0)$ theory described in section 3.1.1 but now applied to $\mathcal{N}=(2,0)$ theories. This corresponds to twisting along the diagonal combination of the Cartan $\mathrm{SO}(2)_{A} \times \mathrm{SO}(2)_{B} \subset \mathrm{SO}(5)_{R}$. The second twist is possible only for $\mathcal{N}=(2,0)$ theories and preserves twice the amount of supersymmetry. It can be viewed as decomposing the R-symmetry group in a block-diagonal form as $\mathrm{SO}(2)_{A} \times \mathrm{SO}(3)_{B} \subset \mathrm{SO}(5)_{R}$ and twisting along $\mathrm{SO}(2)_{A}$.

On Riemann surfaces. We first discuss the universal twist of the $\mathcal{N}=(1,0)$ theory, applied to the $\mathcal{N}=(2,0)$ case. This twist of the maximal theory on $\Sigma_{\mathfrak{g}}$ was considered by Maldacena and Núñez in [20] and we shall refer to it as the $M N \mathcal{N}=1$ twist. The relation (3.5) holds in particular for $\mathcal{N}=(2,0)$ theories, which using $(2.7)$ can be written as

$$
\text { 4d } \mathcal{N}=1: \quad\left(\begin{array}{c}
a_{4 d} \\
c_{4 d}
\end{array}\right)=\frac{(\mathfrak{g}-1)}{384}\left(\begin{array}{cc}
105-33 \\
49 & -1
\end{array}\right)\left(\begin{array}{l}
a_{6 d} \\
c_{6 d}
\end{array}\right) .
$$

This reproduces the central charges for the $4 \mathrm{~d} \mathcal{N}=1$ twisted compactifications of the ADE $\mathcal{N}=(2,0)$ theories derived in $[5,39]$.

The twist along $\mathrm{SO}(2)_{A}$ on $\Sigma_{\mathfrak{g}}$ preserves $4 \mathrm{~d} \mathcal{N}=2$ supersymmetry [20] (see also [49]) and we shall refer to it as the $\mathrm{MN} \mathcal{N}=2$ twist. At the level of line bundles it amounts to the replacement

$$
\mathcal{F}_{R_{A}}^{(6 d)} \rightarrow \mathcal{F}_{R_{0}}^{(4 d)}-\frac{\kappa}{2} t_{\sigma}, \quad \mathcal{F}_{R_{B}}^{(6 d)} \rightarrow \mathcal{F}_{\mathrm{SU}(2)}^{(4 d)}
$$

Performing the twist in the anomaly polynomial and integrating over $\Sigma_{\mathfrak{g}}$ one finds:

$$
4 \mathrm{~d} \mathcal{N}=2: \quad\left(\begin{array}{l}
a_{4 d} \\
c_{4 d}
\end{array}\right)=\frac{(\mathfrak{g}-1)}{72}\left(\begin{array}{l}
21-6 \\
14-2
\end{array}\right)\left(\begin{array}{l}
a_{6 d} \\
c_{6 d}
\end{array}\right) .
$$

Again, this result is compatible with the results in $[5,50]$. In the large $N$ limit the relation between $4 \mathrm{~d}$ and $6 \mathrm{~d}$ conformal anomalies becomes

$$
c_{4 d} \simeq a_{4 d} \simeq \frac{7}{48}(\mathfrak{g}-1) a_{6 d} .
$$

We will derive this relation holographically in section 4.1.2. 
On Kähler four-manifolds. Applying the universal twist of the $\mathcal{N}=(1,0)$ theory on $M_{4}$ to $\mathcal{N}=(2,0)$ theories, the $2 \mathrm{~d}$ central charges are still given by (3.10) which using $(2.7)$ can be written as

$$
\begin{aligned}
c_{r} & =\frac{3}{32}\left(7 a_{6 d}-3 c_{6 d}\right)\left(P_{1}+2 \chi\right)-\frac{1}{24}\left(7 a_{6 d}-4 c_{6 d}\right) P_{1}, \\
c_{l} & =\frac{1}{96}\left(35 a_{6 d}-11 c_{6 d}\right)\left(P_{1}+2 \chi\right)-\frac{1}{8}\left(7 a_{6 d}-4 c_{6 d}\right) P_{1} .
\end{aligned}
$$

This matches the result obtained in [7] for ADE theories (see equation (6.6) there and set $z=\epsilon=0$, which corresponds to the universal twist discussed here).

The second twist, along $\mathrm{SO}(2)_{A}$, amounts to

$$
\mathcal{F}_{R_{A}}^{(6 d)} \rightarrow \mathcal{F}_{R_{0}}^{(2 d)}+\frac{1}{2} t_{1}+\frac{1}{2} t_{2}, \quad \mathcal{F}_{R_{B}}^{(6 d)} \rightarrow \mathcal{F}_{R_{3}}^{(2 d)},
$$

and leads to a $2 \mathrm{~d}(0,4)$ theory with the following anomalies, derived in [7]:

$$
\begin{aligned}
2 \mathrm{~d} \mathcal{N}=(0,4): \quad c_{r} & =\frac{7}{12}\left(P_{1}+2 \chi\right)\left(c_{6 d}-a_{6 d}\right)+\frac{1}{6}\left(P_{1}+3 \chi\right)\left(7 a_{6 d}-4 c_{6 d}\right), \\
k & =c_{r}-c_{l}=\frac{1}{6}\left(P_{1}+\chi\right)\left(7 a_{6 d}-4 c_{6 d}\right) .
\end{aligned}
$$

As discussed in [7] these $2 \mathrm{~d}$ SCFTs do not seem to admit a holographically dual $\mathrm{AdS}_{3}$ description at large $N$. One reason for this might be that the theory obtained in this way does not have a normalizable vacuum, like the $\mathcal{N}=(4,4) \sigma$-model onto the Hitchin moduli space discussed in [51].

Another possible twist on Kähler four-manifolds is to turn on a non-Abelian R-symmetry background. This corresponds to identifying the $\mathrm{U}(2)_{s}$ spin connection with the $\mathrm{U}(2) \subset \mathrm{SO}(4) \subset \mathrm{SO}(5)_{R}$ inside the R-symmetry group. This twist preserves $2 \mathrm{~d}$ $\mathcal{N}=(1,2)$ supersymmetry and was studied in section 6.2 of [7]. We do not discuss it further here.

On product four-manifolds $\boldsymbol{M}_{\mathbf{4}}=\boldsymbol{\Sigma}_{\mathbf{1}} \times \boldsymbol{\Sigma}_{\mathbf{2}}$. When $M_{4}$ is taken to be a product of Riemann surfaces $\Sigma_{1} \times \Sigma_{2}$, with spin connections $\omega_{1,2}$, the holonomy group is reduced to $\mathrm{U}(1)_{\Sigma_{1}} \times \mathrm{U}(1)_{\Sigma_{2}}$ and there is an additional universal twist possible. We take both Riemann surfaces to have negative curvature for simplicity. This was studied in [7]. The twist can be defined by considering the Cartan $\mathrm{SO}(2)_{A} \times \mathrm{SO}(2)_{B}$ subgroup of the $\mathrm{SO}(5)$ R-symmetry and identifying the spin connection $\omega_{1}$ with $\mathrm{SO}(2)_{A}$ and the spin connection $\omega_{2}$ with $\mathrm{SO}(2)_{B}$ (or vice-versa). This is the twist studied in section 3 of [52]. This preserves $2 \mathrm{~d} \mathcal{N}=(2,2)$ supersymmetry and leads to a $2 \mathrm{~d}$ theory with central charges (see equation (5.23) in [7])

$$
2 \mathrm{~d} \mathcal{N}=(2,2): \quad c_{r}=c_{l}=\frac{1}{3}\left(\mathfrak{g}_{1}-1\right)\left(\mathfrak{g}_{2}-1\right)\left(14 a_{6 d}-5 c_{6 d}\right) .
$$

Using the explicit conformal anomalies for the ADE series of $\mathcal{N}=(2,0)$ theories one can show that the $2 \mathrm{~d}$ central charges are an integer multiple of 3 which suggests an interpretation of the $2 \mathrm{~d}$ theory as a nonlinear $\sigma$-model on a Calabi-Yau target space. In the large $N$ limit the central charges become

$$
c_{r}=c_{l} \simeq \frac{7}{4}\left(\mathfrak{g}_{1}-1\right)\left(\mathfrak{g}_{2}-1\right) a_{6 d} .
$$

We will reproduce this holographically in section 4.1.2. 
Other twisted compactifications on four-manifolds leading to $2 \mathrm{~d} \mathcal{N}=(0,2)$ and $\mathcal{N}=$ $(0,1)$ theories, and their supergravity duals, were studied in section 5 of [47] (see also [7]).

On five- and three-manifolds. The twisted compactifications of $6 \mathrm{~d} \mathcal{N}=(2,0)$ theories on the worldvolume of M5-branes on smooth five- and three-manifolds were discussed in [47, 53]. In the former case the whole $\mathrm{SO}(5)_{R} \mathrm{R}$-symmetry group is turned on to implement the twist, which leads to a superconformal quantum mechanics with a single supercharge (see section 3.3 in [47]). In the latter case the twist is obtained by considering $\mathrm{SO}(2) \times \mathrm{SO}(3) \subset$ $\mathrm{SO}(5)_{R}$ and twisting along $\mathrm{SO}(3)$, which leads to a $3 \mathrm{~d} \mathcal{N}=2$ theory (see section 3.1 in [47]). These $3 \mathrm{~d}$ theories were studied also later in [54].

Since we do not have anomalies at our disposal in $3 \mathrm{~d}$ and $1 \mathrm{~d}$, we are limited in our ability to extract universal information about the RG flow across dimensions with field theory techniques. However, the holographic dual description of these RG flows has been constructed in [47], showing that the IR fixed points exist, at least at large $N$. The AdS/CFT dictionary then leads to a universal prediction which would be interesting to test directly in field theory by computing the (partially) twisted partition functions $Z_{S^{1} \times M_{5}}$ and $Z_{S^{3} \times M_{3}}$ in the large $N$ limit and comparing it to the Weyl anomaly coefficients $a_{6 d}, c_{6 d}$. We discuss this further in section 4.1.2 below.

\section{$3.24 d$ SCFTs}

Here we consider $4 \mathrm{~d} \mathcal{N}=1$ and $\mathcal{N}=2$ SCFTs on a smooth Riemann surface $\Sigma_{\mathfrak{g}}$. In the case of $\mathcal{N}=1$ theories there is only one possible topological twist, preserving $\mathcal{N}=(0,2)$ supersymmetry in two dimensions. In the case of $\mathcal{N}=2$ one may also consider twists preserving $\mathcal{N}=(2,2)$ and $\mathcal{N}=(0,4)$. We also comment briefly on twisted compactifications of theories with $\mathcal{N}>2$ as well as compactifications on 3-manifolds.

\subsection{1 $\mathcal{N}=1$}

The partial topological twist of $\mathcal{N}=1$ SCFTs on a compact Riemann surface was discussed in some detail in [9], where an example of the universal relations discussed in this work was presented. At the level of the R-symmetry bundle the topological twist amounts to the shift:

$$
\mathcal{F}_{R}^{(4 d)} \rightarrow \mathcal{F}_{R}^{(2 d)}-\frac{\kappa}{2} t_{\mathfrak{g}}
$$

Integrating the twisted anomaly polynomial $I_{6}^{\text {twisted }}$ over $\Sigma_{\mathfrak{g}}$ and comparing to $(2.1)$ we read off the $2 \mathrm{~d}$ anomaly coefficients. Assuming $9 k_{R R F_{i}}=k_{F_{i}}=0$ for all flavor symmetries $F_{i}{ }^{19}$ one can show that the $2 \mathrm{~d}$ trial central charge is extremized by the UV R-symmetry (see [9] for details) and thus the IR and UV superconformal R-symmetries coincide and one finds the relations

$$
k_{R R}=(\mathfrak{g}-1) k_{R R R}, \quad k=(\mathfrak{g}-1) k_{R} .
$$

\footnotetext{
${ }^{19}$ This assumption is valid for many SCFTs and in even more theories to leading order in $N$, see [9] for a number of examples.
} 
Using (2.5) this can be written as

$$
\left(\begin{array}{l}
c_{r} \\
c_{l}
\end{array}\right)=\frac{16}{3}(\mathfrak{g}-1)\left(\begin{array}{cc}
5 & -3 \\
2 & 0
\end{array}\right)\left(\begin{array}{l}
a_{4 d} \\
c_{4 d}
\end{array}\right) .
$$

In the large $N$ limit this becomes

$$
c_{r} \simeq c_{l} \simeq \frac{32}{3}(\mathfrak{g}-1) a_{4 d} .
$$

Once again, a positive $2 \mathrm{~d}$ central charge requires a negatively curved Riemann surface. We derive this universal relation from holography in section 4.3.1.

\section{$3.2 .2 \mathcal{N}=2$}

For $\mathcal{N}=2$ SCFTs there are more possibilities for twisted compactifications. The Rsymmetry group is $\mathrm{SU}(2) \times \mathrm{U}(1)$, and we denote the Cartan generators by $R_{3}$ and $R_{0}$, respectively. A partial topological twist along $R_{3}$ preserves $2 \mathrm{~d} \mathcal{N}=(2,2)$ supersymmetry, while a twist along $R_{0}$ preserves $\mathcal{N}=(0,4)$ supersymmetry. Following the terminology in [55] we refer to these as the $\alpha$ - and $\beta$-twist, respectively. The twist performed for the $\mathcal{N}=1$ theory in section 3.2.1 above is a particular combination of these, since when one considers the $\mathcal{N}=2$ as a particular example of an $\mathcal{N}=1$ SCFT, the $\mathcal{N}=1$ superconformal R-symmetry is given by the linear combination

$$
R_{\mathcal{N}=1}=\frac{1}{3} R_{0}+\frac{4}{3} R_{3}
$$

We first discuss the $\alpha$-twist. Since this corresponds to a twist along the Cartan of SU(2), it breaks the R-symmetry to $\mathrm{U}(1)_{R_{3}} \times \mathrm{U}(1)_{R_{0}}$, with $R_{3}$ becoming the vector R-symmetry and $R_{0}$ becoming the axial R-symmetry of the $\mathcal{N}=(2,2)$ theory (see, e.g., appendix $\mathrm{F}$ in [56] for details). The anomaly polynomial of the $4 \mathrm{~d}$ theory is given by

$$
I_{6}=\frac{k_{R_{0} R_{0} R_{0}}}{6} c_{1}\left(\mathcal{F}_{R_{0}}\right)^{3}+\frac{k_{R_{0} R_{3} R_{3}}}{2} c_{1}\left(\mathcal{F}_{R_{0}}\right) c_{1}\left(\mathcal{F}_{R_{3}}\right)^{2}-\frac{k_{R_{0}}}{24} c_{1}\left(\mathcal{F}_{R_{0}}\right) p_{1}\left(\mathcal{T}_{4}\right),
$$

where we have used the fact that any trace with an odd number of $R_{3}$ vanishes. The $\alpha$-twist amounts to the replacements:

$$
\mathcal{F}_{R_{3}}^{(4 d)} \rightarrow \mathcal{F}_{r}^{(2 d)}+\mathcal{F}_{l}^{(2 d)}-\frac{\kappa}{2} t_{\mathfrak{g}}, \quad \mathcal{F}_{R_{0}}^{(4 d)} \rightarrow \mathcal{F}_{r}^{(2 d)}-\mathcal{F}_{l}^{(2 d)}
$$

Performing the twist in the anomaly polynomial (3.27) and integrating over $\Sigma_{\mathfrak{g}}$ leads to the $2 \mathrm{~d}$ 't Hooft anomalies

$$
k_{r r}=2(\mathfrak{g}-1) k_{R_{0} R_{3} R_{3}}, \quad k=0 .
$$

Using the relations ${ }^{20}$

$$
k_{R_{0} R_{0} R_{0}}=k_{R_{0}}=48\left(a_{4 d}-c_{4 d}\right), \quad k_{R_{0} R_{3} R_{3}}=2\left(2 a_{4 d}-c_{4 d}\right),
$$

\footnotetext{
${ }^{20}$ These follow from (2.5) and (3.26), and using the fact that any trace with an odd number of $R_{3}$ vanishes.
} 
which are valid for any $4 \mathrm{~d} \mathcal{N}=2$ SCFT, one obtains the $2 \mathrm{~d}$ central charges

$$
2 \mathrm{~d} \mathcal{N}=(2,2): \quad c_{l}=c_{r}=3 k_{r r}=12(\mathfrak{g}-1)\left(2 a_{4 d}-c_{4 d}\right) .
$$

It is interesting to note that the linear combination of $4 \mathrm{~d}$ conformal anomalies, $4\left(2 a_{4 d}-c_{4 d}\right)$, appears in the Shapere-Tachikawa formula [57]

$$
4\left(2 a_{4 d}-c_{4 d}\right)=\sum_{i=1}^{r}\left(2 D\left(O_{i}\right)-1\right) .
$$

Here $r$ is the complex dimension of the Coulomb branch and $D\left(O_{i}\right)$ are the conformal dimensions of the operators $O_{i}$ which parametrize it. Therefore, one can rewrite the $2 \mathrm{~d}$ conformal anomalies in (3.31) as a sum of dimensions of Coulomb branch operators, $c_{l}=$ $c_{r}=3(\mathfrak{g}-1) \sum_{i=1}^{r}\left(2 D\left(O_{i}\right)-1\right)$. This clearly suggests a relation between the $2 \mathrm{~d}(2,2)$ SCFT in the IR and the Coulomb branch of the $4 \mathrm{~d} \mathcal{N}=2 \mathrm{SCFT}$ in the UV. It would be nice to understand this relation more precisely. Let us also note that if the $4 \mathrm{~d} \mathcal{N}=2$ SCFT admits a Lagrangian description in terms of a vector multiplet, with gauge group $G$ of dimension $d_{G}$, then the $2 \mathrm{~d}$ central charges (3.31) can be written as $c_{l}=c_{r}=3(\mathfrak{g}-1) d_{G}$.

If the $4 \mathrm{~d} \mathcal{N}=2 \mathrm{SCFT}$ admits a large $N$ limit (3.31) becomes

$$
c_{l}=c_{r} \simeq 12(\mathfrak{g}-1) a_{4 d} .
$$

We will derive this holographically in section 4.3.2. Finally, we note that for $\mathfrak{g}=0$ the two-dimensional central charge in (3.33) is negative and has the same numerical value as the central charge of the chiral algebra associated to the $4 \mathrm{~d} \mathcal{N}=2$ SCFT following the procedure in [58].

We now discuss the $\beta$-twist, which amounts to the following shift of the U(1) Rsymmetry bundle:

$$
\mathcal{F}_{R_{0}}^{(4 d)} \rightarrow \mathcal{F}_{R}^{(2 d)}-\frac{\kappa}{2} t_{\mathfrak{g}}
$$

The SU(2) R-symmetry of the $4 \mathrm{~d}$ theory is untouched and becomes an $\mathrm{SU}(2)_{R}$ symmetry of the $2 \mathrm{~d} \mathcal{N}=(0,4)$ theory. The central charges of the theory can be computed from an $\mathcal{N}=(0,2)$ subalgebra, whose U(1) R-symmetry is generated by $T=2 T_{3}$, with $T_{3}$ the Cartan of $\mathrm{SU}(2)_{R}$. This gives

$$
2 \mathrm{~d} \mathcal{N}=(0,4): \quad\left(\begin{array}{c}
c_{r} \\
c_{l}
\end{array}\right)=24(\mathfrak{g}-1)\left(\begin{array}{cc}
2 & -1 \\
0 & 1
\end{array}\right)\left(\begin{array}{l}
a_{4 d} \\
c_{4 d}
\end{array}\right) .
$$

We note that the $\beta$-twist has been considered also in [56] and [59]. The expression in (3.35) is the same as the one discussed in appendix A of [59] after setting $\alpha=1$ in that paper.

For $4 \mathrm{~d} \mathcal{N}=2$ SCFTs with a Lagrangian description it is easy to check that for the $\alpha$-twist, $c_{r}=c_{l}$ is an integer multiple of 3 and for the $\beta$-twist $c_{r}$ is an integer multiple of 6 , as expected on general grounds from the (small) $\mathcal{N}=4$ superconformal algebra, see for example [60]. It is natural to conjecture that the IR $2 \mathrm{~d} \mathcal{N}=(2,2)$ SCFT for the $\alpha$-twist can be described in terms of a nonlinear $\sigma$-model on a Calabi-Yau target space. It is certainly desirable to find such a description explicitly. 


\begin{tabular}{|c|c|c|c|c|c|c|c|}
\hline & $H_{0}$ & $H_{1}$ & $H_{2}$ & $D_{4}$ & $E_{6}$ & $E_{7}$ & $E_{8}$ \\
\hline$G_{F}$ & - & $\mathrm{SU}(2)$ & $\mathrm{SU}(3)$ & $\mathrm{SO}(8)$ & $E_{6}$ & $E_{7}$ & $E_{8}$ \\
\hline$h^{\vee}$ & - & 2 & 3 & 6 & 12 & 18 & 30 \\
\hline$c_{4 d}$ & $\frac{11}{30}$ & $\frac{1}{2}$ & $\frac{2}{3}$ & $\frac{7}{6}$ & $\frac{13}{6}$ & $\frac{19}{6}$ & $\frac{31}{6}$ \\
\hline$a_{4 d}$ & $\frac{43}{120}$ & $\frac{11}{24}$ & $\frac{7}{12}$ & $\frac{23}{24}$ & $\frac{41}{24}$ & $\frac{59}{24}$ & $\frac{95}{24}$ \\
\hline$c_{r}^{(\alpha)}=c_{l}^{(\alpha)}$ & $\frac{21}{5}(\mathfrak{g}-1)$ & $5(\mathfrak{g}-1)$ & $6(\mathfrak{g}-1)$ & $9(\mathfrak{g}-1)$ & $15(\mathfrak{g}-1)$ & $21(\mathfrak{g}-1)$ & $33(\mathfrak{g}-1)$ \\
\hline$c_{r}^{(\beta)}$ & $\frac{42}{5}(\mathfrak{g}-1)$ & $10(\mathfrak{g}-1)$ & $12(\mathfrak{g}-1)$ & $18(\mathfrak{g}-1)$ & $30(\mathfrak{g}-1)$ & $42(\mathfrak{g}-1)$ & $66(\mathfrak{g}-1)$ \\
\hline$c_{l}^{(\beta)}$ & $\frac{44}{5}(\mathfrak{g}-1)$ & $12(\mathfrak{g}-1)$ & $16(\mathfrak{g}-1)$ & $28(\mathfrak{g}-1)$ & $52(\mathfrak{g}-1)$ & $76(\mathfrak{g}-1)$ & $124(\mathfrak{g}-1)$ \\
\hline$c_{r}^{(0,2)}$ & $\frac{166}{45}(\mathfrak{g}-1)$ & $\frac{38}{9}(\mathfrak{g}-1)$ & $\frac{44}{9}(\mathfrak{g}-1)$ & $\frac{62}{9}(\mathfrak{g}-1)$ & $\frac{98}{9}(\mathfrak{g}-1)$ & $\frac{134}{9}(\mathfrak{g}-1)$ & $\frac{206}{9}(\mathfrak{g}-1)$ \\
\hline$c_{l}^{(0,2)}$ & $\frac{172}{45}(\mathfrak{g}-1)$ & $\frac{44}{9}(\mathfrak{g}-1)$ & $\frac{56}{9}(\mathfrak{g}-1)$ & $\frac{92}{9}(\mathfrak{g}-1)$ & $\frac{164}{9}(\mathfrak{g}-1)$ & $\frac{236}{9}(\mathfrak{g}-1)$ & $\frac{380}{9}(\mathfrak{g}-1)$ \\
\hline
\end{tabular}

Table 1. Flavor symmetries, dual Coxeter numbers, and $4 \mathrm{~d}$ and $2 \mathrm{~d}$ conformal anomalies for "rank 1 " SCFTs.

As an illustration of our formulas we now consider the well-known "rank 1" $4 \mathrm{~d} \mathcal{N}=2$ SCFTs discussed, for example, around table 1 of [61]. These SCFTs are distinguished by having a one-dimensional Coulomb branch of vacua and we summarize the results for the two-dimensional central charges for the three universal twists we have studied so far, namely the $\alpha$ - and $\beta$-twists discussed above as well as the universal $(0,2)$ twist discussed in section 3.2.1, in table 1. Some comments are in order. First, note that for all theories in table 1, except the $H_{0}$ and $H_{1}$ theories, $c_{r}^{(\alpha)}=c_{l}^{(\alpha)}$ is an integer multiple of 3 and $c_{r}^{(\beta)}$ is an integer multiple of 6 , as should be the case. Theories $H_{0}$ and $H_{1}$ should be treated more carefully in view of the quantization conditions on R-charges discussed around (2.11). The operator with lowest R-charge in the $H_{0}$ theory has the value $r_{H_{0}}=2 / 5$ and the one in the $H_{1}$ theory has $r_{H_{1}}=2 / 3$. It follows from the quantization condition (2.11) that for the $H_{0}$ theory the $\alpha$ - and $\beta$-twist are well defined only when $(\mathfrak{g}-1)$ is an integer multiple of 5 . For the $H_{1}$ theory, by the same analysis, $(\mathfrak{g}-1)$ should be an integer multiple of 3 . These constraints then ensure that for the resulting 2d SCFTs, $c_{r}^{(\alpha)}=c_{l}^{(\alpha)}$ is an integer multiple of 3 and $c_{r}^{(\beta)}$ is an integer multiple of 6 as well. It is also curious to note the following relations between the $2 \mathrm{~d}$ conformal anomalies and the dual Coxeter number of the flavor symmetry group of the four-dimensional theory: ${ }^{21}$

$$
\begin{aligned}
& c_{r}^{(\alpha)}=c_{l}^{(\alpha)}=\left(h^{\vee}+3\right)(\mathfrak{g}-1), \quad c_{r}^{(\beta)}=2\left(h^{\vee}+3\right)(\mathfrak{g}-1), \quad c_{l}^{(\beta)}=4\left(h^{\vee}+1\right)(\mathfrak{g}-1), \\
& c_{r}^{(0,2)}=\frac{2}{9}\left(3 h^{\vee}+13\right)(\mathfrak{g}-1), \quad c_{l}^{(0,2)}=\frac{4}{9}\left(3 h^{\vee}+5\right)(\mathfrak{g}-1) .
\end{aligned}
$$

We note, as a consistency check, that the relation (3.20) for compactifications from $6 \mathrm{~d}$ to $2 \mathrm{~d}$ can be obtained by first compactifying the $6 \mathrm{~d}$ theory to $4 \mathrm{~d}$ by the $\mathrm{MN} \mathcal{N}=2$ twist (3.15) on $\Sigma_{1}$, and subsequently compactifying to 2 d by the $\alpha$-twist (3.31) on $\Sigma_{2}$. Similarly, the 6 d

\footnotetext{
${ }^{21}$ These relations are of course not obeyed for the $H_{0}$ theory in table 1 since this theory does not have a flavor symmetry.
} 
to $2 \mathrm{~d}$ flow relation in (3.19) for the case when the Kähler manifold is a product of Riemann surfaces can be obtained by composing the $\mathrm{MN} \mathcal{N}=2$ twist relation (3.15) with the $\beta$-twist result in (3.35).

Compactification on $\boldsymbol{M}_{\mathbf{3}}$. One may consider the twisted compactification of a $4 \mathrm{~d} \mathcal{N}=2$ SCFT on a three-manifold with holonomy $\mathrm{SO}(3) \simeq \mathrm{SU}(2)$ by turning a background gauge field for the $\mathrm{SU}(2)_{R}$ symmetry. This twist leads to a $1 \mathrm{~d}$ supersymmetric quantum mechanics with two real supercharges. Since we do not have anomalies at our disposal in 1d, we cannot apply the procedure above and we are not able to say much about the properties of the resulting $1 \mathrm{~d}$ theory. However, for $M_{3}=\mathbb{H}^{3}$ the RG flow across dimensions can be constructed holographically (see section 4.3.1), which corresponds to a magnetically charged BPS black hole with an $\mathrm{AdS}_{2}$ near-horizon geometry in asymptotically locally $\mathrm{AdS}_{5}$. This suggests that the quantum mechanical theory in the IR has an emergent conformal symmetry, at least at large $N$. We comment further on this model in section 4.3.1.

\subsubsection{Comments on $\mathcal{N}=3$ and $\mathcal{N}=4$ SCFTs}

It has been recently observed [62-64] that four-dimensional theories with $\mathcal{N}=3$ supersymmetry, but no $\mathcal{N}=4$ supersymmetry, can exist at strong coupling. These have a $\mathrm{U}(3)$ R-symmetry, no flavor symmetries, and it can be shown that $a_{4 d}=c_{4 d}$ [62]. The twisted compactification of these theories on a Riemann surface was considered in [65]. Since the R-symmetry group is contained in that of $\mathcal{N}=4 \mathrm{SYM}$, and the ranks are the same, the possible twists of $\mathcal{N}=3$ theories are contained in those possible for $\mathcal{N}=4 \mathrm{SYM}$, studied in [7]. Viewing an $\mathcal{N}=3$ theory as an $\mathcal{N}=1$ or $\mathcal{N}=2$ theory with additional flavor symmetry, one can apply the twists discussed in sections 3.2.1 and 3.2.2. In particular, the $\alpha$ - and $\beta$-twists of the $\mathcal{N}=2$ theory applied to an $\mathcal{N}=3$ theory lead to a 2 d theory with $\mathcal{N}=(2,2)$ and $\mathcal{N}=(2,4)$ supersymmetry, respectively. Since only the second twist preserves exactly half the amount of supercharges, it is a universal twist of the $\mathcal{N}=3$ theory in the sense defined in the Introduction. ${ }^{22}$ The resulting central charges are given by

$$
2 \mathrm{~d} \mathcal{N}=(2,4): \quad c_{l}=c_{r}=24(\mathfrak{g}-1) a_{4 d},
$$

which is obtained by simply setting $a_{4 d}=c_{4 d}$ in (3.35). Another twist preserving six supercharges arises from taking $\mathrm{U}(3)=\mathrm{SU}(3) \times \mathrm{U}(1)$ and twisting along the $\mathrm{U}(1)$. This leads to a $2 \mathrm{~d}$ theory with $\mathcal{N}=(0,6)$ supersymmetry, $\mathrm{U}(3) \mathrm{R}$-symmetry, and central charges

$$
2 \mathrm{~d} \mathcal{N}=(0,6): \quad c_{l}=c_{r}=\frac{96}{5}(\mathfrak{g}-1) a_{4 d} .
$$

Note that one always obtains a $2 \mathrm{~d}$ theory with no gravitational anomaly, $c_{r}-c_{l}=0$, as a consequence of the absence of gravitational anomalies in $4 \mathrm{~d} \mathcal{N}=3$ theories. For a discussion of other twists of $\mathcal{N}=3$ theories see [65]. We refer the reader to [7] for general twists of $\mathcal{N}=4$ SYM.

\footnotetext{
${ }^{22}$ Although the $\alpha$-twist is regarded (in the sense used here) only as a universal twist of $\mathcal{N}=2$ theories, of course it can also be applied to $\mathcal{N}=3$ theories and the central charges of the resulting $2 \mathrm{~d} \mathcal{N}=(2,2)$ theories are given by $c_{l}=c_{r}=12(\mathfrak{g}-1) a_{4 d}$, which follows by setting $a_{4 d}=c_{4 d}$ in $(3.31)$.
} 


\subsection{SCFTs in odd dimensions}

Based on the results for flows between even-dimensional SCFTs described above, it is natural to ask whether analogous universal relations exist when at least one of the fixed points in the RG flow is an odd-dimensional SCFT. Since there are no 't Hooft and conformal anomalies in odd dimensions, and in view of the $F$-theorem [18], one may look for universal relations involving an appropriately defined partition function, or free energy, of the odd-dimensional SCFT.

The simplest case for which this can be explored by pure field-theoretic methods is the case of three-dimensional $\mathcal{N}=2$ theories placed on a Riemann surface $\Sigma_{\mathfrak{g}}$. The two quantities we wish to compare in this case are the $S^{3}$ partition function of the theory before compactification and the partition function on $S^{1} \times \Sigma_{\mathfrak{g}}$ with a partial topological twist on $\Sigma_{\mathfrak{g}}$. The former, which we denote by $Z_{S^{3}}\left(\Delta_{I}\right)$, was computed by supersymmetric localization in $[17,66]$ and is a function of trial R-charges $\Delta_{I}$ for the theory on $S^{3}$. The latter, which we denote by $Z\left(y_{I}, \mathfrak{n}_{I}\right)$, was computed by localization in [67-69]. It is a function of background magnetic fluxes $\mathfrak{n}_{I}$ specifying the topological twist and flavor fugacities $y_{I}$ and can be interpreted as a twisted index of the $3 \mathrm{~d}$ theory or a Witten index of the $1 \mathrm{~d}$ low-energy theory.

Progress in understanding the relation between these quantities was made recently in $[12,13,19,42,68]$, which we summarize next. Supersymmetric localization shows that both partition functions localize to a matrix model on the Coulomb branch of the theory. Although the resulting matrix models appear to be quite different at finite $N$, it has been observed that for a large class of Chern-Simons matter theories at large $N$ the partition functions are in fact intimately related as follows: ${ }^{23}$

$$
F_{S^{1} \times \Sigma_{\mathfrak{g}}}\left(y_{I}, \mathfrak{n}_{I}\right)=(1-\mathfrak{g})\left[F_{S^{3}}\left(\frac{\Delta_{I}}{\pi}\right)+\sum_{I}\left(\frac{\mathfrak{n}_{I}}{1-\mathfrak{g}}-\frac{\Delta_{I}}{\pi}\right) \frac{\pi}{2} \frac{\partial}{\partial \Delta_{I}} F_{S^{3}}\left(\frac{\Delta_{I}}{\pi}\right)\right],
$$

where we have denoted $F_{S^{1} \times \Sigma_{\mathfrak{g}}}\left(y_{I}, \mathfrak{n}_{I}\right) \equiv-\operatorname{Re} \log Z\left(y_{I}, \mathfrak{n}_{I}\right)$ and one makes the identification $y_{I}=e^{i \Delta_{I}}$, subject to the constraints $\sum_{I} \mathfrak{n}_{I}=2(1-\mathfrak{g})$ and $\sum_{I} \Delta_{I}=2 \pi .^{24}$ The observation made in [19] is that many $3 \mathrm{~d} \mathcal{N}=2$ theories admit a universal topological twist, ${ }^{25}$ which amounts to setting the flux parameters as $\mathfrak{n}_{I}=\frac{(1-\mathfrak{g})}{\pi} \bar{\Delta}_{I}$, where $\bar{\Delta}_{I}$ are the exact R-charges of the UV theory obtained by extremizing $F_{S^{3}}$ with respect to the $\Delta_{I}$, as instructed in [17]. One can then see that the twisted index (3.39) is also extremized for the same values of $\Delta_{I}$ and thus the R-charges in the UV and IR coincide. Evaluating both sides of (3.39) for these values of $\Delta_{I}, \mathfrak{n}_{I}$, one thus finds the simple large $N$ universal relation

$$
F_{S^{1} \times \Sigma_{\mathfrak{g}}}=(1-\mathfrak{g}) F_{S^{3}} .
$$

This is the first example we encounter of a universal relation of the form (1.3). It would be interesting to determine the exact role of subleading orders in $N$ in this relation. As

\footnotetext{
${ }^{23}$ This was first shown for $\Sigma_{\mathfrak{g}}=S^{2}$ in [13], for a generic genus in the case of ABJM theory in [68], and for a larger class of quiver theories and generic Riemann surface in [19].

${ }^{24}$ The constraint on $\sum_{I} \Delta_{I}$ is rather subtle and requires a detailed large $N$ analysis of the matrix model. See [19] for a more detailed discussion.

${ }^{25}$ This is consistent only if the exact R-charges in the SCFT are rational. Although this is not a problem for many $3 \mathrm{~d} \mathcal{N}=2$ theories, there are an infinite number of examples for which this issue arises. See [19, 42] for a more detailed discussion.
} 
we discuss in section 4.4, this twisted compactification is described holographically by a magnetically charged black hole in $\mathrm{AdS}_{4}$, whose entropy at large $N$ is computed by $F_{S^{1} \times \Sigma_{\mathfrak{g}}}$. This gives further motivation for studying subleading corrections to this quantity.

Let us comment on 3d SCFTs with $\mathcal{N}>2$. Clearly, all such theories can be viewed as $\mathcal{N}=2$ theories and one can readily apply the results discussed above. However, as in the case of the $\alpha$ - and $\beta$-twists of $4 \mathrm{~d}$ theories discussed in section 3.2.2, one may wonder if twisted compactifications with enhanced supersymmetry exhibit any universal properties. For $\mathcal{N}=3$ SCFTs the R-symmetry is $\mathrm{SO}(3)$ and thus the only topological twist available is the universal one. We note that if the $\mathcal{N}=3$ theory at hand admits a Lagrangian description (and perhaps even more generally), the R-charges are rational and thus the universal twist can always be performed for some appropriate value of the genus, $\mathfrak{g}>1$. For $3 \mathrm{~d} \mathcal{N}=4$ SCFTs the situation is more interesting. ${ }^{26}$ These theories have $\mathrm{SU}(2)_{C} \times \mathrm{SU}(2)_{H}$ R-symmetry and thus admit more general topological twists on $\Sigma_{\mathfrak{g}}$. The $\mathcal{N}=2$ universal twist preserves two real supercharges and amounts to turning on a magnetic flux along the Cartan generator of the diagonal SU(2) subgroup of the R-symmetry group. There are, however, two other twists which preserve four real supercharges and are obtained by turning on a magnetic flux along the Cartan generator of either $\mathrm{SU}(2)_{C}$ or $\mathrm{SU}(2)_{H}$. These twisted compactifications were recently studied in [70]. One can then study the topologically twisted index for both of these twists using the results of [67-69]. In order to make a connection with the universal relations derived above, and ultimately with holography, we are interested in the large $N$ limit of these indices. It turns out, however, that the topologically twisted index is trivial to leading order in $N$ for both topological twists, i.e. the corresponding free energy vanishes. ${ }^{27}$ It would certainly be interesting to explore these two twisted compactifications further and understand whether the topologically twisted indices obey any type of universal relation at finite $N$.

Let us briefly comment on the case $d=5$. The only superconformal algebra in five dimensions is $F(4)$, with eight supercharges and an $\mathrm{SU}(2)_{R}$ R-symmetry. The twisted compactification of such SCFTs on a Riemann surface $\Sigma_{\mathfrak{g}}$ by twisting along the Cartan of $\mathrm{SU}(2)_{R}$ leads to a $3 \mathrm{~d}$ SCFT with $\mathcal{N}=2$ supersymmetry. The twisted compactification on a three-manifold $M_{3}$ by a non-Abelian twist along the full $\mathrm{SU}(2)_{R}$ leads to a $2 \mathrm{~d}$ SCFT with $\mathcal{N}=(1,1)$ supersymmetry. Finally, one may also consider the compactification on a Kähler four-manifold, leading to supersymmetric quantum mechanical theories. The holographic description of these RG flows across dimensions was considered in [71, 72], which we discuss in section 4.2. While the partition function of 5d SCFTs on various fivemanifolds has been studied extensively in the literature, much less is known for partially twisted compactifications. ${ }^{28}$ In view of the simple holographic relations between the UV and IR free energies (or conformal anomalies in the case of $M_{3}$ ) that we uncover in section 4.2, it would be interesting to explore this further in field theory.

Finally, let us point out that for RG flows in which $d$ is even and $p$ is odd (or vice versa) it is tempting to look for universal relations among free energies and conformal anomalies.

\footnotetext{
${ }^{26}$ We refrain from discussing SCFTs with $\mathcal{N}>4$ here.

${ }^{27} \mathrm{We}$ are grateful to Alberto Zaffaroni for informing us of this result.

${ }^{28}$ See however [73] for a localization calculation of $5 \mathrm{~d}$ theories on $S^{3} \times \Sigma_{\mathfrak{g}}$ with a topological twist on $\Sigma_{\mathfrak{g}}$.
} 
While we have not studied this in field theory, and are not aware of any discussion in the literature, ${ }^{29}$ the holographic analysis in section 4 provides evidence that such universal relations exist at large $N$. This certainly deserves further study.

\subsection{Comments on Hofman-Maldacena bounds}

The Hofman-Maldacena (HM) bounds are bounds on the ratio $a_{4 d} / c_{4 d}$ in four-dimensional CFTs derived from energy positivity constraints. These were first proposed in [74] (see also $[57,75]$ ), and were recently proven in [76] using conformal bootstrap methods. For supersymmetric theories these bounds read:

$$
\mathcal{N}=1: \quad \frac{1}{2} \leq \frac{a_{4 d}}{c_{4 d}} \leq \frac{3}{2}, \quad \mathcal{N}=2: \quad \frac{1}{2} \leq \frac{a_{4 d}}{c_{4 d}} \leq \frac{5}{4}
$$

It is interesting to study how various values of $a_{4 d} / c_{4 d}$ are mapped to six and two dimensions by the RG flows across dimensions discussed above.

Consider first $4 \mathrm{~d} \mathcal{N}=1$ theories obtained by the twisted compactification of $6 \mathrm{~d} \mathcal{N}=$ $(2,0)$ theories on a Riemann surface. The four- and six-dimensional central charges are related by (3.13). One can further compactify to $2 \mathrm{~d}$ by the universal twist preserving $2 \mathrm{~d}$ $\mathcal{N}=(0,2)$ supersymmetry. The two- and four-dimensional central charges are then related by (3.24). In figure 1 we show how various values are mapped across dimensions. We observe that $6 \mathrm{~d}$ ADE theories (shaded region) always lead to $4 \mathrm{~d} \mathcal{N}=1$ theories satisfying the HM bounds, with the upper HM bound in $4 \mathrm{~d}$ saturated when $a_{6 d} / c_{6 d}=1$. Since the value $\frac{a_{4 d}}{c_{4 d}}=\frac{3}{2}$ is associated to a free $4 \mathrm{~d} \mathcal{N}=1$ vector multiplet it is natural to conjecture that six-dimensional $\mathcal{N}=(2,0)$ SCFTs with $a_{6 d}=c_{6 d}$ flow to a free gauge theory upon this twisted compactification. Upon further compactification to $2 \mathrm{~d}$ we note that the positivity constraint $c_{r} \geq 0$ imposed by $2 \mathrm{~d}$ unitarity requires $a_{6 d} / c_{6 d} \geq 3 / 7{ }^{30}$ Notice also that the two-dimensional unitarity bound, $c_{r} \geq 0$, translates into the bound $a_{4 d} / c_{4 d} \geq 3 / 5$ for $4 \mathrm{~d}$ $\mathcal{N}=1$ SCFTs. A free $\mathcal{N}=1$ chiral multiplet saturates the lower bound $a_{4 d} / c_{4 d}=1 / 2$ but we are not aware of any other unitary $4 \mathrm{~d} \mathcal{N}=1$ SCFT in the range $1 / 2 \leq a_{4 d} / c_{4 d}<3 / 5$. A natural question arising from this observation is whether one can use two-dimensional unitarity, in combination with the universal RG flows from $4 \mathrm{~d}$ to $2 \mathrm{~d}$, to derive a stronger lower bound on $a_{4 d} / c_{4 d}$ than the one appearing in (3.41), in the case of interacting theories.

Consider now $4 \mathrm{~d} \mathcal{N}=2$ theories obtained by twisted compactification of $6 \mathrm{~d} \mathcal{N}=(2,0)$ theories on a Riemann surface, whose central charges are related by (3.15). Once again, we see that $6 \mathrm{~d}$ ADE theories lead to $4 \mathrm{~d}$ theories satisfying the HM bounds, with the upper HM bound in $4 \mathrm{~d}$ saturated when $a_{6 d} / c_{6 d}=1$. Again this naturally suggests that a $6 \mathrm{~d}$ $\mathcal{N}=(2,0)$ theory with $a_{6 d}=c_{6 d}$ leads to the theory of a free $\mathcal{N}=2$ vector multiplet upon this twisted compactification. One may further reduce to $2 \mathrm{~d}$, either by the $\alpha$-twist (3.31),

\footnotetext{
${ }^{29}$ See [41] for a related but distinct discussion on the connection between conformal anomalies and sphere free energies for theories in different dimensions.

${ }^{30}$ It is curious to note that this lower bound on $a_{6 d} / c_{6 d}$ appeared also in [77] in a different context. We also note that one may repeat our analysis for $4 \mathrm{~d} \mathcal{N}=1$ SCFTs arising from twisted compactifications of $6 \mathrm{~d}$ $(1,0)$ theories and their further compactification to $2 \mathrm{~d}$, in which case one finds $\alpha \geq 0$ for $2 \mathrm{~d}$ unitarity. Since these 6d SCFTs have three independent conformal anomaly coefficients it is less clear how to conjecture possible HM-type bounds on conformal anomalies and we refrain from doing so.
} 


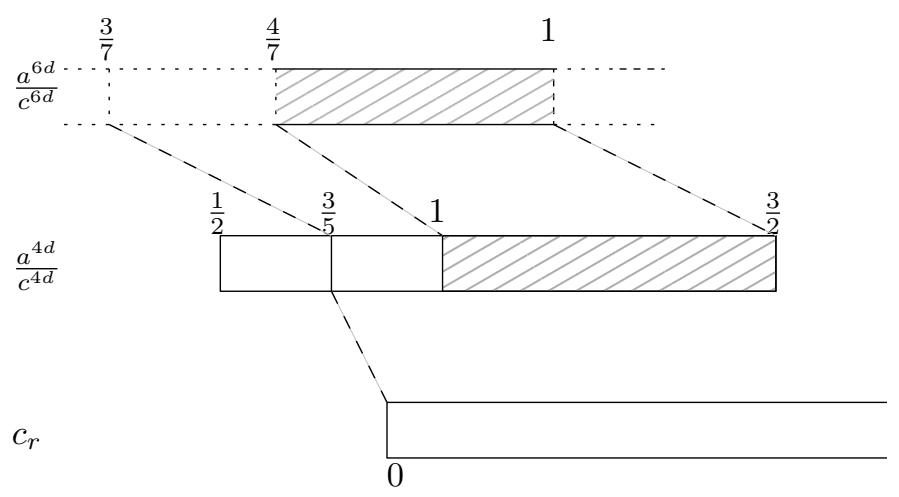

Figure 1. Mapping of various values of $a / c$ under (3.13) from $6 \mathrm{~d} \mathcal{N}=(2,0)$ to a $4 \mathrm{~d} \mathcal{N}=1$ theory, as well as the flow from $4 \mathrm{~d}$ to $2 \mathrm{~d}$ obeying $(3.24)$ and preserving $\mathcal{N}=(0,2)$ supersymmetry. Positivity of $c_{r}$ in $2 \mathrm{~d}$ is mapped into the lower bound $3 / 7$ in $6 \mathrm{~d}$.

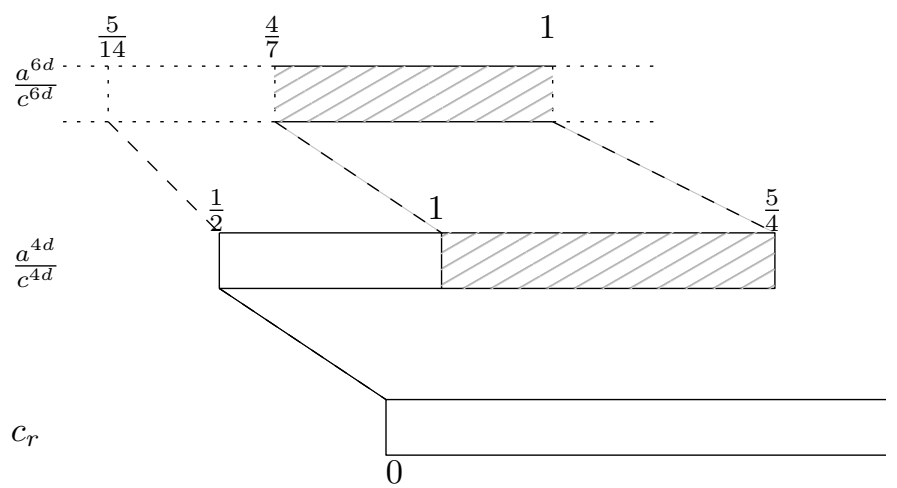

Figure 2. Mapping of various values of $a / c$ under (3.15) from $6 \mathrm{~d} \mathcal{N}=(2,0)$ to a $4 \mathrm{~d} \mathcal{N}=2$ theory. The map to $2 \mathrm{~d}$, either by the $\alpha$-twist (3.31) or the $\beta$-twist (3.35), relates the lower HM bound in $4 \mathrm{~d}$ to the $2 \mathrm{~d}$ unitarity bound $c_{r} \geq 0$ ( $c_{l} \geq 0$ is automatically satisfied).

or the $\beta$-twist (3.35). In both cases the lower HM bound in $4 \mathrm{~d}$ is mapped to $c_{r}=0$ in $2 \mathrm{~d}$, as shown in figure 2 . This is very intriguing and suggests a relation between unitarity in two dimensions and the bounds in (3.41). As noted in section 2.2, the $6 \mathrm{~d} \mathcal{N}=(2,0)$ ADE theories ${ }^{31}$ have conformal anomaly coefficients obeying $4 / 7<a_{6 d} / c_{6 d}<1$. Upon the $\mathcal{N}=2$ twisted compactification on $\Sigma_{\mathfrak{g}}$ and using (3.15) this implies that 4 d class $\mathcal{S}$ SCFTs have central charges obeying $a_{4 d} / c_{4 d}>1$. Note also that this class of $4 \mathrm{~d}$ SCFTs arise from M5-branes wrapping $\Sigma_{\mathfrak{g}}$ and have well-known holographic dual descriptions [20, 50]. This observation is in conflict with the recent results in [78] where the authors argued for a positivity bound on the Gauss-Bonnet coupling in higher-curvature gravitational theories. In particular, the results in [78] imply that for CFTs with a weakly coupled holographic dual, such as the theories at hand, one should find $a_{4 d} / c_{4 d}<1$. It would be interesting to understand the reasons for this inconsistency.

\footnotetext{
${ }^{31}$ These are the only currently known $\mathcal{N}=(2,0)$ SCFTs in six dimensions.
} 


\section{Gauged supergravity}

We now turn to the holographic description of the universal RG flows described in section 3. As we have argued, when the topological twist involves only the stress-energy tensor multiplet the RG flow is universal and is not sensitive to the details of the particular theory being compactified. General results in superconformal representation theory show (see for example $[79,80]$ for a recent account) that the stress-energy multiplet for SCFTs in $d=3,4,5,6$ has an operator spectrum exactly dual to the fields in the gravity multiplet of a gauged supergravity in one dimension higher. This suggests that the holographic description of universal RG flows across dimensions should be captured entirely by the dynamics of the gravity multiplet in the appropriate gauged supergravity. We refer to a supergravity theory containing only the gravity multiplet as minimal gauged supergravity. ${ }^{32}$ Motivated by the analysis of section 3 , in this section we discuss supersymmetric solutions of minimal gauged supergravities in 4, 5, 6, and 7 dimensions describing universal $\mathrm{RG}$ flows.

We impose the following conditions on the supergravity solutions. At asymptotic infinity the metric should approach an asymptotically locally $\operatorname{AdS}_{d+1}$ background, with an $\mathbb{R}^{p} \times M_{d-p}$ boundary. In the interior of the geometry there should be another asymptotic region, where the metric approaches $\mathrm{AdS}_{p+1} \times M_{d-p}$. This is captured by an Ansatz of the form

$$
d s_{d+1}^{2}=e^{2 f(r)} d s_{\mathbb{R}^{p+1}}^{2}+e^{2 g(r)} d s_{M_{d-p}}^{2},
$$

with the following asymptotics:

$$
\begin{aligned}
r \rightarrow \infty: & e^{2 f(r)}=\frac{1}{r^{2}}, & e^{2 g(r)}=\frac{1}{r^{2}}, \\
r \rightarrow 0: & e^{2 f(r)}=\frac{e^{2 f_{0}}}{r^{2}}, & e^{2 g(r)}=e^{2 g_{0}},
\end{aligned}
$$

where $r$ is the holographic direction, with the UV corresponding to $r \rightarrow \infty$ and the IR to $r \rightarrow 0$, and $f_{0}, g_{0}$ are constants determined by the supergravity BPS equations. In addition, there must be a nontrivial graviphoton gauge field $A_{\mu}^{R}$, proportional to the spin connection on $M_{d-p}$. This gauge field flux, which is the holographic manifestation of the topological twist, ensures that the solution preserves a certain amount of supersymmetry. In theories with more than 8 supercharges, there are also scalars in the gravity multiplet which generically also acquire a nontrivial radial profile as a function of $r$. The entire supergravity solution can be viewed as a $(p-1)$-dimensional BPS black brane in $\operatorname{AdS}_{d+1}$ carrying a magnetic charge under the graviphoton $A_{\mu}^{R}$. We emphasize that the magnetic charge of the black brane is fixed to a unique value by supersymmetry. ${ }^{33}$ Since for the purposes of our discussion it suffices to focus on the $\mathrm{AdS}_{p+1}$ and $\mathrm{AdS}_{d+1}$ asymptotics of the solution, we will not present the full interpolating domain wall explicitly. ${ }^{34}$

\footnotetext{
${ }^{32}$ This might not be standard terminology but we employ it here to emphasize that there are no other multiplets apart from the gravity multiplet.

${ }^{33}$ Generalizations including general charges under additional vector multiplets (dual to flavor symmetries in the field theory) are possible. However, these solutions will not be universal, as argued in the Introduction.

${ }^{34}$ The full radial dependence of the metric functions and possible scalar fields can be found analytically in some examples but in general one has to resort to numerical integration of the BPS equations.
} 
The careful reader may have noticed a sleight of hand in our discussion. When one performs the topologically twisted reduction in the field theory there is no guarantee that the IR dynamics of the QFT will be governed by an interacting SCFT with a weakly coupled holographic dual. We have allowed for this possibility in our discussion below, by allowing the IR region of the gravitational domain wall to have a metric different from $\operatorname{AdS}_{p+1}$ and studying the BPS equations in this more general setup. All such solutions, however, turn out to be singular, suggesting that the corresponding twisted compactifications have some "pathological" behavior in the IR. For instance, the IR theory could become free, have a non-normalizable vacuum, or accidental global symmetries.

We should emphasize that there is a vast literature on constructing domain wall solutions in gauged supergravity. An important vantage point on these solutions was offered by the work of Maldacena and Núnez [20], where twisted compactifications of $6 \mathrm{~d} \mathcal{N}=(2,0)$ theories and $4 \mathrm{~d} \mathcal{N}=4 \mathrm{SYM}$ on Riemann surfaces were studied holographically, and shown to correspond to supergravity backgrounds of the kind discussed above. Our main goal in this section is not to find new supergravity solutions, but rather to collect and organize various backgrounds scattered throughout the literature, and to interpret them in the context of the universal flows discussed in section 3. In particular, a crucial point in our story is that these universal black brane solutions can be embedded in ten- or eleven-dimensional supergravity in infinitely many ways (see section 4.5 for a more detailed discussion). This is precisely the statement of universality of the solutions. Different embeddings of these supergravity backgrounds in string or M-theory describe the twisted compactification of different SCFTs, but the universal relations for field theory observables discussed in section 3 always hold, regardless of the details of the SCFTs. This point of view not only establishes the existence of RG flows across dimensions for a large class of SCFTs (at least to leading order in $N$ ), but can also be a powerful tool in counting the microstates of infinite families of black branes. This was made explicit for the case of black holes in $\mathrm{AdS}_{4}$ in [19], following the approach of [12]. It is natural to expect that the observations made here will be useful in generalizing these results to even larger classes of black branes in different dimensions.

Before describing the solutions of interest, let us collect some expressions for the holographic evaluation of central charges and free energies, which will be used repeatedly below. For odd-dimensional AdS solutions the central charges of the dual even-dimensional CFTs are given by [81, 82]:

$$
c_{r}=c_{l}=\frac{3 L_{\mathrm{AdS}_{3}}}{2 G_{N}^{(3)}}, \quad a_{4 d}=c_{4 d}=\frac{\pi L_{\mathrm{AdS}_{5}}^{3}}{8 G_{N}^{(5)}}, \quad a_{6 d}=\frac{4}{7} c_{6 d}=\frac{3 \pi^{2} L_{\mathrm{AdS}_{7}}^{5}}{7 G_{N}^{(7)}}
$$

Here $G_{N}^{(d+1)}$ is the Newton constant in $(d+1)$ dimensions and $L_{\mathrm{AdS}_{d+1}}$ is the length scale associated with the given AdS vacuum. In the case of even-dimensional AdS solutions we are interested in the renormalized value of the on-shell action, which is mapped holographically to the free energy of the dual SCFT placed on a round sphere. In the case of $\mathrm{AdS}_{4}$ and $\mathrm{AdS}_{6}$ vacua one finds the following expressions for the sphere free energy (see for example [83]):

$$
F_{S^{3}}=\frac{\pi L_{\mathrm{AdS}_{4}}^{2}}{2 G_{N}^{(4)}}, \quad F_{S^{5}}=-\frac{\pi^{2} L_{\mathrm{AdS}_{6}}^{4}}{3 G_{N}^{(6)}} .
$$


One should not be bothered by the minus sign in $F_{S^{5}}$ since the "proper" monotonically decreasing quantity under RG flow in an odd dimension $d$ is conjectured to be given by $(-1)^{(d-1) / 2} \log Z_{S^{d}}$ [18], where $Z_{S^{d}}$ is the partition function on $S^{d}$ and we define $F_{S^{d}}=$ $-\log Z_{S^{d}}$.

In the case of $\mathrm{AdS}_{2}$ vacua, describing the near-horizon geometry of BPS black holes, the main quantity of interest will be the Bekenstein-Hawking entropy of the black hole. As shown explicitly in [19] for black holes in $\mathrm{AdS}_{4}$ this is intimately related to the renormalized gravitational free energy of the solution. ${ }^{35}$

Finally, all the solutions we discuss are locally asymptotic to the $\mathrm{AdS}_{d+1}$ vacuum of the gauged supergravity theory. The length scale of this vacuum is set by the value of the scalar potential of the theory at its $\mathrm{AdS}_{d+1}$ critical point, $\left.V\right|_{\phi_{\text {crit }}}$. This value in turn sets the cosmological constant scale, which is related to the value of the gauge coupling constant in the supergravity theory. In our conventions the radius, $L_{\mathrm{AdS}_{d+1}}$, of $\mathrm{AdS}_{d+1}$ is given by

$$
\left.V\right|_{\phi_{\text {crit }}}=-\frac{d(d-1)}{L_{\mathrm{AdS}_{d+1}}^{2}}
$$

We choose a normalization in which $L_{\mathrm{AdS}_{d+1}}=1$, thus fixing the value of the gauged supergravity coupling constant to a particular value, as determined by (4.6).

\section{$4.17 \mathrm{~d}$ supergravity}

In this section, we provide the holographic description of RG flows from $6 \mathrm{~d} \mathcal{N}=(1,0)$ and $\mathcal{N}=(2,0)$ SCFTs to lower-dimensional SCFTs by the twisted compactifications described in section 3.1. The relevant supergravity theories are the $7 \mathrm{~d} \mathcal{N}=2$ gauged supergravity of [84] (see also [85] for some details) and the $7 \mathrm{~d} \mathcal{N}=4$ gauged supergravity of [86], respectively.

\subsection{1 $\mathcal{N}=2$}

The bosonic content of this minimally supersymmetric theory is the graviton, an $\mathrm{SU}(2)$ graviphoton, a real scalar $\lambda$, and a three-form potential $C_{3}$. In the solutions of interest the gauge field is excited only along the Cartan of $\mathrm{SU}(2)$ and $C_{3}=0$, in which case the bosonic Lagrangian reads

$$
\begin{aligned}
\mathcal{L} & =R-20 * d \lambda \wedge d \lambda-V(\lambda) * 1-e^{-4 \lambda} * F \wedge F \\
V & =\frac{1}{2} m^{2}\left(-8 e^{4 \lambda}-8 e^{-6 \lambda}+e^{-16 \lambda}\right)
\end{aligned}
$$

where $m$ is the gauge coupling constant. The $\mathrm{AdS}_{7}$ vacuum of the theory corresponds to the extremum of the potential at $\lambda=0$, where it takes the value $\left.V\right|_{\lambda=0}=-15 \mathrm{~m}^{2} / 2$. Comparing to (4.6) we set $m=2$ in what follows to normalize $L_{\mathrm{AdS}_{7}}=1$. Universal holographic RG flow solutions in this theory were studied in [26, 27].

\footnotetext{
${ }^{35}$ It would be interesting to study this relation for more general AdS black holes/branes in various dimensions.
} 
$\mathbf{A d S}_{5}$ vacua. We are interested in supersymmetric solutions of the form

$$
\begin{aligned}
d s^{2} & =e^{2 f(r)}\left(-d t^{2}+d r^{2}+d z_{1}^{2}+d z_{2}^{2}+d z_{3}^{2}\right)+e^{2 g(r)} d s_{\Sigma_{\mathfrak{g}>1}}^{2}, \\
F & =\frac{1}{8} d \operatorname{vol}_{\Sigma_{\mathfrak{g}>1}} .
\end{aligned}
$$

Note the coefficient in the graviphoton field strength $F$ (and thus the corresponding magnetic charge of the solution) is fixed, anticipating the constraint imposed by supersymmetry. ${ }^{36}$ We focus on $\mathfrak{g}>1$ since there are no regular solutions for $\mathfrak{g}=0,1$. To obtain the BPS equations we note that when the gauge field points along the Cartan of $\mathrm{SU}(2)$, as in the Ansatz above, the minimal theory can be obtained as a subsector of the $\mathrm{U}(1)^{2}$ truncation of the $7 \mathrm{~d} \mathrm{SO}(5)$ maximally supersymmetric gauged supergravity. ${ }^{37}$ General RG flows in this $\mathrm{U}(1)^{2}$ truncation were studied in [5]. We may thus borrow the BPS equations for an Ansatz of the form (4.8) from this reference, ${ }^{38}$ which read

$$
\begin{aligned}
& e^{-f} f^{\prime}=-2 e^{-f} \lambda^{\prime}-e^{-8 \lambda} \\
& e^{-f} g^{\prime}=-2 e^{-f} \lambda^{\prime}+\frac{1}{4} e^{-2 g-2 \lambda}+e^{-8 \lambda} \\
& e^{-f} \lambda^{\prime}=-\frac{2}{5}\left(e^{-8 \lambda}-e^{2 \lambda}\right)+\frac{1}{40} e^{-2 g-2 \lambda}
\end{aligned}
$$

where a prime denotes differentiation with respect to $r$. These equations describe the full RG flow from $\mathrm{AdS}_{7}$ to $\mathrm{AdS}_{5} \times \Sigma_{\mathfrak{g}}$. At the $\mathrm{AdS}_{5}$ fixed point the metric functions take the form (4.3), with

$$
e^{f_{0}}=e^{8 \lambda_{0}}, \quad e^{g_{0}}=\frac{1}{2} e^{3 \lambda_{0}}, \quad e^{10 \lambda_{0}}=\frac{3}{4},
$$

where $\lambda_{0}$ is the value of the scalar field at the fixed point.

The $4 \mathrm{~d}$ central charges associated to this solution are readily computed from (4.4), giving:

$$
a_{4 d}=c_{4 d}=\frac{\pi e^{2 g_{0}+3 f_{0}} \operatorname{vol}\left(\Sigma_{\mathfrak{g}}\right)}{8 G_{N}^{(7)}}=\frac{63}{512}(\mathfrak{g}-1) a_{6 d}
$$

This result is in perfect agreement with the large $N$ limit of the field theory calculation in (3.6). This is a nice consistency check that the supergravity solution at hand is dual to the universal RG flow across dimensions discussed around (3.6).

The entire solution corresponds to a BPS 3-brane in asymptotically locally $\mathrm{AdS}_{7}$, whose entropy density is given in terms of IR field theory data by the general relation (B.6) and can be expressed in terms of UV field theory data by the universal relation (4.11).

\footnotetext{
${ }^{36}$ This will be the case for all the solutions discussed in this paper.

${ }^{37}$ More precisely, the bosonic content of this truncation is two gauge fields $A_{\mu}^{(1)}, A_{\mu}^{(2)}$, a three form potential $C_{\mu \nu \rho}$ and two scalar fields $\lambda_{1}, \lambda_{2}$. To obtain the minimal theory one must set $A_{\mu}^{(1)}=A_{\mu}^{(2)} \equiv A_{\mu}$ and $\lambda_{1}=\lambda_{2} \equiv \lambda$. One can check, for instance, that the Lagrangian and supersymmetry variations obtained in this way are consistent with those of [87].

${ }^{38}$ These can also be obtained from the BPS equations derived in [20].
} 
$\mathbf{A d S}_{\mathbf{3}}$ vacua. The Ansatz in this case is

$$
\begin{aligned}
d s^{2} & =e^{2 f(r)}\left(-d t^{2}+d z^{2}+d r^{2}\right)+e^{2 g(r)} d s_{M_{4}}^{2}, \\
F & =-\frac{1}{8} \omega_{M_{4}},
\end{aligned}
$$

where $d s_{M_{4}}^{2}$ is a constant-curvature metric on a Kähler-Einstein manifold $M_{4}$, with Kähler form $\omega_{M_{4}}$, normalized such that

$$
R_{\mu \nu}^{(4)}=-g_{\mu \nu}^{(4)},
$$

where we took $M_{4}$ to be negatively curved (only this case leads to regular supergravity solutions). A solution of this form within $7 \mathrm{~d}$ maximally supersymmetric supergravity was found in [47] (see equation (4.12) there and recall we set $m=2$ ). As argued above, for this Ansatz the same BPS equations hold in minimal supergravity, which read: ${ }^{39}$

$$
\begin{aligned}
e^{-f} f^{\prime} & =-\frac{1}{5}\left(4 e^{-2 \lambda}+e^{8 \lambda}\right)-\frac{3}{160} e^{-4 \lambda-4 g}-\frac{1}{10} e^{-2 g+2 \lambda}, \\
e^{-f} g^{\prime} & =-\frac{1}{5}\left(4 e^{-2 \lambda}+e^{8 \lambda}\right)+\frac{1}{80} e^{-4 \lambda-4 g}+\frac{3}{20} e^{-2 g+2 \lambda}, \\
e^{-f} \lambda^{\prime} & =-\frac{2}{5}\left(e^{-2 \lambda}-e^{8 \lambda}\right)+\frac{1}{160} e^{-4 \lambda-4 g}-\frac{1}{20} e^{-2 g+2 \lambda} .
\end{aligned}
$$

At the $\mathrm{AdS}_{3}$ fixed point the metric functions take the form (4.3), with

$$
e^{5 f_{0}}=\frac{1}{24}, \quad e^{10 g_{0}}=\frac{27}{2^{16}}, \quad e^{10 \lambda_{0}}=\frac{4}{3},
$$

where $\lambda_{0}$ is the value of the scalar field at the fixed point.

The corresponding $2 \mathrm{~d}$ central charges can be computed from (4.4) and read:

$$
c_{r}=c_{l}=\frac{3 e^{f_{0}+4 g_{0}} \operatorname{vol}\left(M_{4}\right)}{2 G_{N}^{(7)}}=\frac{21 \operatorname{vol}\left(M_{4}\right)}{256 \pi^{2}} a_{6 d}
$$

This result is in nice agreement with the large $N$ limit of the field theory calculation in (3.12).

The entire solution describes a BPS 1-brane asymptotic to $\mathrm{AdS}_{7}$, whose entropy density can be expressed in terms of UV SCFT data by combining (B.5) and (4.16).

$\mathbf{A d S}_{4}$ vacua. The relevant $\mathrm{AdS}_{4}$ solution in $7 \mathrm{~d}$ minimal supergravity was found in [88] (see also the later work [53]) and preserves only two Poincaré and two superconformal supercharges, i.e. the dual 3d SCFT has $\mathcal{N}=1$ supersymmetry. The universal nature of this solution was also emphasized in [27]. We do not discuss this further here.

\subsection{2 $\mathcal{N}=4$}

The maximally supersymmetric $\mathrm{SO}(5)$ gauged supergravity of [86] is the relevant setting for studying twisted compactifications of $6 \mathrm{~d} \mathcal{N}=(2,0)$ SCFTs holographically. For topological twists involving an Abelian subgroup of $\mathrm{SO}(5)$ it is sufficient to restrict to the $\mathrm{U}(1)^{2}$

\footnotetext{
${ }^{39}$ These can also be obtained as a special case of the general flows studied in [7] (see equation (6.16) in that reference and set $\lambda_{1}=\lambda_{2} \equiv \lambda$ and $\left.z=0\right)$.
} 
truncation of the $\mathrm{SO}(5)$ gauged supergravity with bosonic fields the metric, two Abelian gauge fields $A^{(1)}, A^{(2)}$ in the Cartan of $\mathrm{SO}(5)$, and two real scalars $\lambda_{1}, \lambda_{2}$. This truncation was studied in [87] (see equations (2.5), (2.12), and (2.13) there for the bosonic Lagrangian and supersymmetry variations).

One may embed the $7 \mathrm{~d} \mathcal{N}=2$ gauged supergravity into the $\mathcal{N}=4$ theory, and the solutions discussed in the previous section are also solutions of the maximally supersymmetric theory, with the additional fields set to zero. From the field theory perspective this corresponds to applying the universal twist of general $6 \mathrm{~d} \mathcal{N}=(1,0)$ theories to the special case of $\mathcal{N}=(2,0)$ theories. In this section, we discuss additional twists possible for $\mathcal{N}=(2,0)$ theories and their gravity duals.

$\mathbf{A d S}_{5}$ vacua The solution describing the twisted compactification of the $6 \mathrm{~d} \mathcal{N}=(2,0)$ theory to a $4 \mathrm{~d} \mathcal{N}=2$ theory was first found by Maldacena and Núñez in [20]. For completeness, we reproduce the answer here, following the notation and conventions in [5]. The Ansatz is

$$
\begin{aligned}
d s^{2} & =e^{2 f(r)}\left(-d t^{2}+d r^{2}+d z_{1}^{2}+d z_{2}^{2}+d z_{3}^{2}\right)+e^{2 g(r)} d s_{\Sigma_{\mathfrak{g}>1}}^{2}, \\
F^{(1)} & =-\frac{1}{4} d \operatorname{vol}_{\Sigma_{1}}, \quad F^{(2)}=0 .
\end{aligned}
$$

At the $\mathrm{AdS}_{5}$ fixed point the metric functions take the form (4.3), with (see equation (3.8) in [5]):

$$
e^{2 f_{0}}=2 e^{2 g_{0}}=\frac{1}{2^{4 / 5}}, \quad e^{2 \lambda_{1}^{(0)}}=\frac{1}{2} e^{2 \lambda_{2}^{(0)}}=\frac{1}{2^{3 / 5}},
$$

where $\lambda_{1,2}^{(0)}$ are the values of the scalars at the fixed point.

Using (4.4), the corresponding $4 \mathrm{~d}$ central charges read

$$
a_{4 d}=c_{4 d}=\frac{\pi e^{3 f_{0}+2 g_{0}} \operatorname{vol}\left(\Sigma_{\mathfrak{g}}\right)}{8 G_{N}^{(7)}}=\frac{7}{48}(\mathfrak{g}-1) a_{6 d}
$$

which exactly reproduces the large $N$ field theory result (3.16).

The entire solution describes a BPS 3-brane in $\mathrm{AdS}_{7}$ and its entropy density is readily expressed in terms of UV data by combining (B.6) and (4.19).

AdS $_{3}$ vacua. The compactification on $M_{4}=\Sigma_{1} \times \Sigma_{2}$, with both Riemann surfaces of negative curvature, preserving $2 \mathrm{~d} \mathcal{N}=(2,2)$ supersymmetry is given by (see equation $(5.26)$ and appendix $\mathrm{G}$ of [7])

$$
\begin{aligned}
& d s^{2}=e^{2 f(r)}\left(-d t^{2}+d r^{2}+d z^{2}\right)+e^{2 g_{1}(r)} d s_{\Sigma_{1}}^{2}+e^{2 g_{2}(r)} d s_{\Sigma_{2}}^{2}, \\
& F^{A}=-\frac{1}{4} d \operatorname{vol}_{\Sigma_{1}}, \quad F^{B}=-\frac{1}{4} d \operatorname{vol}_{\Sigma_{2}} .
\end{aligned}
$$

At the $\mathrm{AdS}_{3}$ fixed point the metric functions take the form (4.3), with

$$
e^{2 f_{0}}=e^{2 g_{1}^{(0)}}=e^{2 g_{2}^{(0)}}=\frac{1}{4}
$$

and the scalars vanish: $\lambda_{1}=\lambda_{2}=0$. 
The corresponding $2 \mathrm{~d}$ central charges, computed from (4.4), read:

$$
c_{r}=c_{l}=\frac{3 e^{f_{0}+2 g_{1}^{(0)}+2 g_{2}^{(0)}}}{2 G_{N}^{(7)}} \operatorname{vol}\left(\Sigma_{1}\right) \operatorname{vol}\left(\Sigma_{2}\right)=\frac{7}{4}\left(\mathfrak{g}_{1}-1\right)\left(\mathfrak{g}_{2}-1\right) a_{6 d}
$$

which exactly reproduces the field theory result (3.21).

The entire solution describes a BPS 1-brane asymptotic to $\mathrm{AdS}_{7}$, whose entropy density is given in terms of UV data by combining (B.5) and (4.22).

$\mathbf{A d S}_{4}$ vacua. $\mathrm{AdS}_{4}$ vacua arising from M5-branes wrapping special Lagrangian 3-cycles were found in section 3.1 of [47] (see also [53]). These describe the twisted compactification of $6 \mathrm{~d} \mathcal{N}=(2,0)$ theories on three-manifolds, discussed at the end of section 3.1.2. For $M_{3}$ an Einstein space of negative constant curvature, there is an $\mathrm{AdS}_{4}$ fixed point, for which the metric functions take the form (4.3), with

$$
e^{f_{0}}=\frac{e^{4 \lambda_{0}}}{2}, \quad e^{2 g_{0}}=\frac{e^{8 \lambda_{0}}}{8} \quad e^{10 \lambda_{0}}=2
$$

where $\lambda_{0}$ is the value of the only nontrivial scalar field at the fixed point.

The $S^{3}$ free energy of the corresponding $\mathrm{AdS}_{4}$ solution follows from (4.5) and is given by

$$
F_{S^{3}}=\frac{\pi e^{2 f_{0}+3 g_{0}} \operatorname{vol}\left(M_{3}\right)}{2 G_{N}^{(7)}}=\frac{7 \operatorname{vol}\left(M_{3}\right)}{96 \pi \sqrt{2}} a_{6 d}
$$

where in the last equality we used (4.4). The entire solution describes a BPS 2-brane asymptotic to $\mathrm{AdS}_{7}$, whose entropy density is given in terms of UV data by combining (B.8) and (4.24).

$\mathbf{A d S}_{2}$ vacua. $\mathrm{AdS}_{2}$ vacua arising from M5-branes wrapping special Lagrangian 5-cycles $M_{5}$ were found in section 3.3 of [47]. These describe the twisted compactification of $6 \mathrm{~d}$ $\mathcal{N}=(2,0)$ theories on five-manifolds discussed at the end of section 3.1.2. Regular solutions to the supergravity BPS equations were found for $S^{5}$ and $\mathbb{H}^{5}$ with an Einstein metric of normalized curvature $\kappa=1$ and $\kappa=-1$, respectively. ${ }^{40}$ The supergravity scalars vanish and the metric functions are given by

$$
e^{2 f_{0}}=\frac{(2-\kappa)^{2}}{64}, \quad e^{2 g_{0}}=\frac{(2-\kappa)}{16} .
$$

With this at hand the black hole entropy corresponding to this near horizon $\mathrm{AdS}_{2}$ solution can be written as

$$
S_{\mathrm{BH}}=\frac{e^{5 g_{0}} \operatorname{vol}\left(M_{5}\right)}{4 G_{N}^{(7)}}=\frac{7(2-\kappa)^{5 / 2} \operatorname{vol}\left(M_{5}\right)}{3 \times 2^{12} \pi^{2}} a_{6 d}
$$

where in the last equality we made use of (4.4). It would be nice to reproduce this result by a field theory calculation.

\footnotetext{
${ }^{40} \mathrm{It}$ is curious to note that this is the only example of a regular universal domain wall solution with a positively curved metric on the compactification manifold $M_{d-p}$.
} 


\section{$4.26 \mathrm{~d}$ supergravity}

There is a unique six-dimensional gauged supergravity theory with a supersymmetric $\mathrm{AdS}_{6}$ vacuum that can be constructed out of the gravity multiplet. This was done by Romans in [89]. The theory has 16 supercharges and the $\mathrm{AdS}_{6}$ vacuum is invariant under the supergroup $F(4)$, which is also the unique superconformal group in $5 \mathrm{~d}$. The bosonic field content of the theory is given by the graviton $g_{\mu \nu}, \mathrm{SU}(2)$ gauge potentials $A_{\mu}^{I}$, an Abelian one-form potential $A_{\mu}$, a two-index tensor gauge field $B_{\mu \nu}$, and a scalar $\phi$. The two-form "eats" the one-form $A_{\mu}$ and becomes massive, which can be implemented by choosing $A_{\mu}=$ 0 . This bosonic content mimics precisely the bosonic operators in the energy-momentum multiplet of 5d SCFTs. In particular, the $\mathrm{SU}(2)$ gauge field $A_{\mu}^{I}$ is dual to the R-current. The fermionic field content is four gravitinos $\psi_{\mu i}$ and four gauginos $\chi_{i}$ with a symplectic Majorana condition. To implement the topological twist of interest here we only need to use the metric, the gauge field, and the scalar. Therefore we will set the two-form $B_{\mu \nu}=0$ from now on. Using the conventions summarized in [72], the bosonic Lagrangian reads ${ }^{41}$

$$
\begin{aligned}
e^{-1} \mathcal{L} & =R-4\left(\partial_{\mu} \varphi\right)^{2}-e^{-2 \varphi} F^{I \mu \nu} F_{\mu \nu}^{I}-V(\varphi), \\
V(\varphi) & =\frac{1}{2}\left(m^{2} e^{-6 \varphi}-\bar{g}^{2} e^{2 \varphi}-4 \bar{g} m e^{-2 \varphi}\right),
\end{aligned}
$$

where $\bar{g}$ is the $\mathrm{SU}(2)$ coupling constant and $m$ is a mass parameter associated to $B_{\mu \nu}$. Depending on the signs of $\bar{g}, m$, or if any of these parameters vanishes, this Lagrangian actually describes five different theories. Here we are interested in the case $\bar{g}>0, m>0$, which corresponds to the theory labelled $\mathcal{N}=4^{+}$in [89]. Further setting $\bar{g}=3 \mathrm{~m}$ the theory admits an $\mathrm{AdS}_{6}$ vacuum with $F(4)$ symmetry, corresponding to the extremum of the potential at $\varphi=0$ where it takes the value $\left.V\right|_{\varphi=0}=-10 \mathrm{~m}^{2}$. Comparing this to (4.6) we set $m=\sqrt{2}$ in what follows to normalize $L_{\mathrm{AdS}_{6}}=1$.

Our main interest here is in the solutions of this $F(4)$ gauged supergravity describing the twisted compactification of 5d SCFTs on two-, three-, and four-manifolds, as briefly discussed at the end of section 3.3. The corresponding $\mathrm{AdS}_{4}, \mathrm{AdS}_{3}$, and $\mathrm{AdS}_{2}$ vacua, which we review below, were constructed in [71, 72]. We mostly follow the conventions in [72], where the relevant BPS equations are written (see equations (5.12) there). We denote by $D=5-p$ the dimension of the compactification manifold. The supergravity equations imply the metric on this manifold should be Einstein, whose normalized curvature we denote by $\kappa$. With this notation the BPS equations read

$$
\begin{aligned}
e^{-f} \varphi^{\prime} & =\frac{1}{4}\left(3 e^{\varphi}-3 e^{-3 \varphi}-\frac{\kappa D}{6} e^{-2 g-\varphi}\right), \\
e^{-f} g^{\prime} & =-\frac{1}{4}\left(3 e^{\varphi}+e^{-3 \varphi}+\frac{\kappa(8-D)}{6} e^{-2 g-\varphi}\right), \\
e^{-f} f^{\prime} & =-\frac{1}{4}\left(3 e^{\varphi}+e^{-3 \varphi}-\frac{\kappa D}{6} e^{-2 g-\varphi}\right) .
\end{aligned}
$$

Next, we describe the asymptotic behavior of solutions to these equations for the relevant values of $D$.

\footnotetext{
${ }^{41}$ Compared to [72] we have defined $\varphi_{\text {here }}=\frac{1}{\sqrt{2}} \phi_{\text {there }}$ and denote the coupling constant by $\bar{g}$ instead of $g$.
} 
$\mathbf{A d S}_{4}$ vacua. The twisted compactification of a $5 \mathrm{~d}$ SCFT on a Riemann surface to a $3 \mathrm{~d}$ $\mathcal{N}=2$ SCFT is described by a solution of the form

$$
\begin{aligned}
d s^{2} & =e^{2 f(r)}\left(-d t^{2}+d z_{1}^{2}+d z_{2}^{2}+d r^{2}\right)+e^{2 g(r)} d s_{\Sigma_{\mathfrak{g}}}^{2}, \\
F^{I} & =\frac{2 \kappa}{3 \sqrt{2}} \delta^{I 3} d \operatorname{vol}_{\Sigma_{\mathfrak{g}}} .
\end{aligned}
$$

Setting $D=2$ in (4.28) one finds that for $\kappa=-1$ there is an $\mathrm{AdS}_{4}$ fixed point, with metric functions of the form (4.3), with

$$
e^{f_{0}}=\frac{2}{3} e^{-\varphi_{0}}, \quad e^{g_{0}}=\frac{\sqrt{2}}{3} e^{-\varphi_{0}}, \quad e^{-4 \varphi_{0}}=\frac{3}{2},
$$

with $\varphi_{0}$ the value of the scalar field at the $\mathrm{AdS}_{4}$ fixed point.

The free energy on $S^{3}$ of the $3 \mathrm{~d}$ SCFT dual to this $\mathrm{AdS}_{4}$ vacuum is computed from (4.5) by evaluating the on-shell action and reads

$$
F_{S^{3}}=\frac{\pi e^{2 f_{0}+2 g_{0}} \operatorname{vol}\left(\Sigma_{\mathfrak{g}}\right)}{2 G_{N}^{(6)}}=-\frac{8}{9}(\mathfrak{g}-1) F_{S^{5}}
$$

This universal relation is a nontrivial prediction of supergravity which would be interesting to derive directly in field theory.

The entire solution describes a 2-brane asymptotic to $\mathrm{AdS}_{6}$, whose entropy density is given in terms of UV data by combining (B.8) and (4.31).

$\mathbf{A d S}_{\mathbf{3}}$ vacua. The solution describing the twisted compactification on a hyperbolic threemanifold $M_{3}=\mathbb{H}^{3} / \Gamma$ was constructed in section 3.1 of [71] and is of the form

$$
\begin{aligned}
d s^{2} & =e^{2 f(r)}\left(-d t^{2}+d z^{2}+d r^{2}\right)+e^{2 g(r)} d s_{\mathbb{H}^{3}}^{2}, \\
A^{1} & =-\frac{1}{3 \sqrt{2}} \frac{d x_{1}}{x_{2}}, \quad A^{3}=-\frac{1}{3 \sqrt{2}} \frac{d x_{3}}{x_{2}}, \quad A^{2}=0 .
\end{aligned}
$$

where $d s_{\mathbb{H}^{3}}^{2}=\frac{1}{x_{2}^{2}}\left(d x_{1}^{2}+d x_{2}^{2}+d x_{3}^{2}\right)$ is the metric on hyperbolic space, which we quotient by an appropriate discrete group $\Gamma$. Setting $D=3$ and $\kappa=-1$ in (4.28) one finds an $\mathrm{AdS}_{3}$ fixed point, where the metric functions take the form (4.3), with

$$
e^{f_{0}}=\frac{1}{2} e^{-\varphi_{0}}, \quad e^{g_{0}}=\frac{1}{\sqrt{6}} e^{-\varphi_{0}}, \quad e^{-4 \varphi_{0}}=2,
$$

with $\varphi_{0}$ the value of the scalar at the horizon.

This twisted compactification preserves $2 \mathrm{~d} \mathcal{N}=(1,1)$ supersymmetry and thus we have fewer technical tools to study the IR SCFT. However, we can compute holographically the central charge of the $2 \mathrm{~d}$ theory using (4.4) and find

$$
c_{r}=c_{l}=\frac{3 e^{f_{0}+3 g_{0}} \operatorname{vol}\left(M_{3}\right)}{2 G_{N}^{(6)}}=-\sqrt{\frac{3}{2}} \frac{\operatorname{vol}\left(M_{3}\right)}{4 \pi^{2}} F_{S^{5}}
$$

where in the last equality we have used (4.5).

The entire solution describes a 1-brane asymptotic to $\mathrm{AdS}_{6}$, whose entropy density is given in terms of UV data by the formulas (B.5) and (4.34). 
$\mathbf{A d S}_{\mathbf{2}}$ vacua. $\mathrm{AdS}_{2}$ vacua were found in [72], corresponding to twisted compactification of $5 \mathrm{~d}$ SCFTs on four-manifolds $M_{4}$. Since the gauge field in the supergravity theory is only $\mathrm{SU}(2)$, this restricts the possible 4-manifolds one may consider. One option is for $M_{4}$ to be Kähler. ${ }^{42}$ Then, setting $D=4$ in (4.28) the BPS equations dictate that $\kappa=-1$ and one finds an $\mathrm{AdS}_{2}$ fixed point, where the metric functions take the form (4.3), with

$$
e^{f_{0}}=\frac{1}{3} e^{-\varphi_{0}}, \quad e^{g_{0}}=\frac{1}{3} e^{-\varphi_{0}}, \quad e^{-4 \varphi_{0}}=3,
$$

with $\varphi_{0}$ the value of the scalar at the horizon.

The entire spacetime is a BPS black hole asymptotic to $\mathrm{AdS}_{6}$, with entropy

$$
S_{\mathrm{BH}}=\frac{e^{4 g_{0}} \operatorname{vol}\left(M_{4}\right)}{4 G_{N}^{(6)}}=-\frac{\operatorname{vol}\left(M_{4}\right)}{36 \pi^{2}} F_{S^{5}}
$$

where in the last equality we used (4.5). This is yet another universal prediction from holography which would be interesting to test with field theory methods, by comparing the partition function on $S^{1} \times M_{4}$ (with a universal topological twist on $M_{4}$ ) and the partition function on $S^{5}$ at large $N$.

\section{$4.35 d$ supergravity}

We now proceed to study $\mathrm{AdS}_{3}$ and $\mathrm{AdS}_{2}$ vacua of $5 \mathrm{~d} \mathcal{N}=2$ and $\mathcal{N}=4$ minimal gauged supergravity. These describe universal twisted compactifications of $4 \mathrm{~d} \mathcal{N}=1$ and $\mathcal{N}=2$ SCFTs on Riemann surfaces and three manifolds.

\subsection{1 $\mathcal{N}=2$}

This theory has 8 supercharges and bosonic field content the metric $g_{\mu \nu}$ and a U(1) gauge field $A_{\mu}$ dual to the stress-energy tensor and the R-current in the dual $4 \mathrm{~d} \mathcal{N}=1$ SCFT. $^{43}$ Here we follow the conventions of $[7,20]$ in which the Lagrangian reads (see equation (46) in [20])

$$
e^{-1} \mathcal{L}=R+12-\frac{3}{4} F_{\mu \nu}^{2}+\frac{1}{4} e^{-1} \epsilon^{\mu \nu \alpha \beta \rho} F_{\mu \nu} F_{\alpha \beta} A_{\rho},
$$

where the cosmological constant is normalized such that $L_{\mathrm{AdS}_{5}}=1$.

$\mathbf{A d S}_{3}$ vacua. We are interested in BPS solutions of the supergravity theory with $\mathrm{AdS}_{5}$ asymptotics and near-horizon geometry $\mathrm{AdS}_{3} \times \Sigma_{\mathfrak{g}}$. These describe the universal flow from $4 \mathrm{~d} \mathcal{N}=1$ SCFTs to $2 \mathrm{~d} \mathcal{N}=(0,2)$ SCFTs discussed in $[8,9]$ and reviewed in section 3.2.1.

The Ansatz for the metric and gauge field are

$$
\begin{aligned}
d s^{2} & =e^{2 f(r)}\left(-d t^{2}+d z^{2}+d r^{2}\right)+e^{2 g(r)} d s_{\Sigma_{\mathfrak{g}}}^{2}, \\
F & =\frac{\kappa}{3} d \operatorname{vol}_{\Sigma_{\mathfrak{g}}}
\end{aligned}
$$

\footnotetext{
${ }^{42}$ Another option is for $M_{4}$ to be a co-associative cycle in a non-compact $G_{2}$ holonomy manifold, in which case the metric has the same form; see section 5.1 of [72].

${ }^{43}$ This theory can be obtained from the well-studied STU model of gauged supergravity by setting the vector multiplet fields to zero.
} 
and the resulting BPS equations read: ${ }^{44}$

$$
\begin{aligned}
e^{-f} f^{\prime} & =-1+\frac{\kappa}{6} e^{-2 g} \\
e^{-f} g^{\prime} & =-1-\frac{\kappa}{3} e^{-2 g} .
\end{aligned}
$$

The solution to these equations with the required asymptotics, which exists only for $\kappa=-1$, describes a magnetically charged BPS black string in $\mathrm{AdS}_{5}$ and was discussed in [90]. The entire domain wall solution preserves 2 real supercharges, which is enhanced as usual to 4 supercharges at the horizon.

For the purpose of computing the central charge of the IR 2d SCFT, we focus on the $\mathrm{AdS}_{3}$ fixed point of (4.39), where the metric functions take the form (4.3), with

$$
e^{f_{0}}=2, \quad e^{2 g_{0}}=\frac{2}{3} .
$$

The $2 \mathrm{~d}$ central charges can then be easily be found to read

$$
c_{r}=c_{l}=\frac{3 e^{f_{0}+2 g_{0}} \operatorname{vol}\left(\Sigma_{\mathfrak{g}}\right)}{2 G_{N}^{(5)}}=\frac{32}{3}(\mathfrak{g}-1) a_{4 d}
$$

reproducing the universal field theory result in (3.25).

The entropy density of the black string can be written in terms of the data of the $4 \mathrm{~d}$ UV SCFT by combining (B.5) with (4.41).

$\mathbf{A d S}_{\mathbf{2}}$ vacua. Since minimal gauged supergravity in 5 d contains only a U(1) gauge field and the holonomy group of a generic Riemannian three-manifold is $\mathrm{SO}(3)$, we do not expect to find supersymmetric $\mathrm{AdS}_{2}$ vacua. However, it is possible to construct such vacua in the $5 \mathrm{~d} \mathcal{N}=4$ theory, which we review next.

\subsection{2 $\mathcal{N}=4$}

The $5 \mathrm{~d} \mathcal{N}=4$ gauged supergravity theory has 16 supercharges. The bosonic field content is the graviton, $g_{\mu \nu}, \mathrm{SU}(2) \times \mathrm{U}(1)$ gauge fields $A_{\mu}^{I}, I=1,2,3$, and $A_{\mu}$, two antisymmetric tensor fields $B_{\mu \nu}^{1,2}$, and a scalar $\phi$. This structure nicely corresponds to the one of the stress-energy tensor multiplet in the dual $4 \mathrm{~d} \mathcal{N}=2$ SCFT. Depending on the values of the $\mathrm{U}(1)$ and $\mathrm{SU}(2)$ gauge coupling constants $g_{1}, g_{2}$, respectively, there are three different gauged supergravity theories and found in [91]. Here we are interested in the unique theory, denoted by $\mathcal{N}=4^{+}$in [91], for which there is a supersymmetric $\mathrm{AdS}_{5}$ critical point. In the notation of [91] this theory is obtained by setting $\bar{g} \equiv g_{2}=\sqrt{2} g_{1}$. Here we are interested in supersymmetric solutions of this theory which describe the topological twisted

\footnotetext{
${ }^{44}$ As noted above $\mathcal{N}=2$ gauged supergravity can be obtained by setting $A_{\mu}^{1}=A_{\mu}^{2}=A_{\mu}^{3} \equiv A_{\mu}$ and $\phi_{1}=\phi_{2}=0$ in the $\mathrm{U}(1)^{3}$ truncation of $\mathcal{N}=8$ gauged supergravity. Thus it is useful to make contact with previous references on this well-studied model. Setting $a_{1}=a_{2}=a_{3}=\frac{1}{3}$ and $\phi_{1}=\phi_{2}=0$ in equation (3.20) in [7] leads to the BPS equations in (4.39). These also coincide with equations (65-68) in [20] after setting $a=b=c=\frac{1}{3}$ and $\varphi=0$.
} 
compactifications discussed in section 3.2.2 and thus we set the two-form fields $B_{\mu \nu}^{1,2}$ to zero to find the following bosonic Lagrangian ${ }^{45}$

$$
e^{-1} \mathcal{L}=R-\frac{1}{2}\left(D_{\mu} \phi\right)^{2}-e^{-\frac{4 \phi}{\sqrt{6}}} F^{\mu \nu} F_{\mu \nu}-\frac{1}{2} e^{\frac{2 \phi}{\sqrt{6}}} F^{\mu \nu I} F_{\mu \nu}^{I}+\frac{1}{2} \bar{g}^{2}\left(2 e^{\frac{\phi}{\sqrt{6}}}+e^{-\frac{2 \phi}{\sqrt{6}}}\right) .
$$

Normalizing the scale of $\mathrm{AdS}_{5}$ as $L_{\mathrm{AdS}_{5}}=1$ requires to set the value of the potential at the critical point $\phi=0$ to be $\left.V\right|_{\phi=0}=-\frac{3 \bar{g}^{2}}{2}=-12$ and hence $\bar{g}=2 \sqrt{2}$. We use this normalization from now on and for convenience define $\phi \equiv \sqrt{6} \varphi$.

$\mathbf{A d S}_{3}$ vacua. We are after a solution describing the universal flow from a $4 \mathrm{~d} \mathcal{N}=2$ theory to a $2 \mathrm{~d} \mathcal{N}=(2,2)$ theory. As discussed in section 3.2 .2 this is obtained by a topological twist along the Cartan of $\mathrm{SU}(2)$. Thus, we are after $\mathrm{AdS}_{3}$ solutions where only the gauge field along the Cartan of $\mathrm{SU}(2)$ is turned on and the $\mathrm{U}(1)$ gauge field vanishes. The Ansatz for the metric and gauge fields is thus

$$
\begin{aligned}
d s^{2} & =e^{2 f(r)}\left(-d t^{2}+d z^{2}+d r^{2}\right)+e^{2 g(r)} d s_{\Sigma_{\mathfrak{g}}}^{2}, \\
F^{I} & =\frac{\kappa}{2} \delta^{I 3} d \operatorname{vol}_{\Sigma_{\mathfrak{g}}}, \quad F=0 .
\end{aligned}
$$

The corresponding BPS equations read

$$
\begin{aligned}
e^{-f} f^{\prime} & =-\frac{1}{3}\left(2 e^{-\varphi}-e^{2 \varphi}\right)+\frac{\kappa}{6} e^{-2 g+\varphi}, \\
e^{-f} g^{\prime} & =-\frac{1}{3}\left(2 e^{-\varphi}-e^{2 \varphi}\right)-\frac{\kappa}{3} e^{-2 g+\varphi}, \\
e^{-f} \varphi^{\prime} & =-\frac{2}{3}\left(e^{-\varphi}+e^{2 \varphi}\right)+\frac{\kappa}{6} e^{-2 g+\varphi} .
\end{aligned}
$$

The solution to these equations with the desired asymptotics, which exists only for $\kappa=$ -1 , was constructed in [91]. ${ }^{46}$ At the $\mathrm{AdS}_{3}$ fixed point the metric functions take the form (4.3) with

$$
e^{2 f_{0}}=e^{2 g_{0}}=\frac{1}{2^{4 / 3}}, \quad e^{\varphi}=2^{1 / 3} .
$$

The entire domain wall solution preserves 4 real supercharges which are enhanced to 8 supercharges at the horizon. The corresponding $2 \mathrm{~d}$ central charges are given by

$$
c_{r}=c_{l}=\frac{3 L_{\mathrm{AdS}_{3}}}{2 G_{N}^{(3)}}=\frac{3 e^{f_{0}+2 g_{0}} \operatorname{vol}\left(\Sigma_{\mathfrak{g}}\right)}{2 G_{N}^{(5)}}=12(\mathfrak{g}-1) a_{4 d}
$$

which nicely matches the large $N$ field theory result (3.33). The entropy density of this supersymmetric black string solution in terms of UV SCFT data is obtained by combining (B.5) and (4.46).

\footnotetext{
${ }^{45}$ We obtain this by setting all fermionic fields and antisymmetric tensor fields to zero in the Lagrangian (2.14) in [91] and sending $g_{\mu \nu} \rightarrow-g_{\mu \nu}$ to change to a "mostly plus" signature. We have also rescaled the scalar field $\phi_{\text {there }}=\frac{1}{2} \phi_{\text {here }}$ and the $\mathrm{SU}(2)$ gauge field $F_{\text {here }}^{I}=\sqrt{2} F_{\text {there }}^{I}$.

${ }^{46}$ Setting $x=\frac{1}{2}$ in [91] corresponds to turning off the U(1) gauge field, leaving a nontrivial gauge field for the Cartan of $\mathrm{SU}(2)_{R}$ only; see equation (4.5) in [91].
} 
We note that the BPS equations (4.44) coincide with the BPS equations describing the twisted compactification of $\mathcal{N}=4 \mathrm{SYM}$ on a Riemann surface preserving $2 \mathrm{~d} \mathcal{N}=(2,2)$ supersymmetry, discussed in [20] (see equations (14)-(16) there). ${ }^{47}$ In that reference the flow was described within the $\mathrm{U}(1)^{3}$ truncation of $\mathcal{N}=8$ supergravity, which consists of the metric $g_{\mu \nu}$, three Abelian gauge fields $\mathcal{A}_{\mu}^{1,2,3}$ in the Cartan of $\mathrm{SO}(6)$ and two neutral scalars $\phi_{1}$ and $\phi_{2}$. The solution described above corresponds to a solution with $\mathcal{A}_{\mu}^{1}=\mathcal{A}_{\mu}^{2}$, $\mathcal{A}_{\mu}^{3}=\phi_{2}=0$, and identifying the remaining scalar $\phi_{1}=\sqrt{6} \varphi$, in which case one can see that the Lagrangians and supersymmetry transformations coincide. ${ }^{48}$ These results are in harmony with the fact that the $\mathcal{N}=4^{+}$Romans supergravity theory can be obtained also as a truncation of the $5 \mathrm{~d} \mathrm{SO}(6)$ maximally supersymmetric gauged supergravity of [92].

The twisted compactification corresponding to the $\beta$-twist discussed in section 3.2.2 is realized in the $\mathcal{N}=4^{+}$supergravity by turning on magnetic flux for the $\mathrm{U}(1)$ gauge field, $A_{\mu}$, and switching off the $\mathrm{SU}(2)$ gauge field flux, $A_{\mu}^{I}$. However, one can show that an Ansatz with this field configuration leads to a singular supergravity flow solution which does not flow to an $\mathrm{AdS}_{3}$ vacuum in the IR. This suggests that the dual $2 \mathrm{~d} \mathcal{N}=(0,4)$ theory in the IR has some pathology, for example an accidental symmetry or a non-normalizable vacuum state.

$\mathbf{A d S}_{\mathbf{2}}$ vacua. An $\mathrm{AdS}_{2}$ vacuum in $5 \mathrm{~d} \mathcal{N}=4$ minimal gauged supergravity was found [93]. This describes the twisted compactification of a $4 \mathrm{~d} \mathcal{N}=2$ theory on $M_{3}=\mathbb{H}^{3} / \Gamma$, with $\mathbb{H}^{3}$ three-dimensional hyperbolic space and $\Gamma$ a finite subgroup of its isometry group. Since the structure group of the three-manifold is $\mathrm{SO}(3)$, in order to implement the topological twist one has to switch off the Abelian gauge field in the supergravity theory. The Ansatz for the metric and $\mathrm{SU}(2)$ gauge field is

$$
\begin{aligned}
d s^{2} & =e^{2 f(r)}\left(-d t^{2}+d r^{2}\right)+e^{2 g(r)} d s_{\mathbb{H}^{3}}^{2}, \\
A^{1} & =\frac{1}{2 \sqrt{2}} \cosh \phi d \theta, \quad A^{2}=\frac{1}{2 \sqrt{2}} \cos \theta d \nu, \quad A^{3}=-\frac{1}{2 \sqrt{2}} \sin \theta \cosh \phi d \nu,
\end{aligned}
$$

where $d s_{\mathbb{H}^{3}}^{2}=d \phi^{2}+\sinh ^{2} \phi\left(d \theta^{2}+\sin ^{2} \theta d \nu^{2}\right)$. The corresponding BPS equations were derived in [93] (see equation (56) there), which we reproduce here for completeness:

$$
\begin{aligned}
e^{-f} f^{\prime} & =-\frac{1}{2} e^{\varphi-2 g}-\frac{1}{3}\left(2 e^{-\varphi}+e^{2 \varphi}\right), \\
e^{-f} g^{\prime} & =\frac{1}{2} e^{\varphi-2 g}-\frac{1}{3}\left(2 e^{-\varphi}+e^{2 \varphi}\right), \\
e^{-f} \varphi^{\prime} & =-\frac{1}{2} e^{\varphi-2 g}-\frac{2}{3}\left(e^{-\varphi}-e^{2 \varphi}\right) .
\end{aligned}
$$

As discussed in [93] (see equation (42) there) at the $\mathrm{AdS}_{2}$ fixed point the metric functions take the form (4.3) with

$$
e^{2 g_{0}}=\frac{1}{4^{1 / 3}}, \quad e^{2 f_{0}}=\frac{1}{4^{4 / 3}}, \quad e^{3 \varphi_{0}}=4,
$$

\footnotetext{
${ }^{47}$ These can also be obtained by setting $a_{1}=a_{2}=-\frac{\kappa}{2}$ and $a_{3}=0$ in equation (3.20) of [7].

${ }^{48}$ One can check that setting $\mathcal{A}_{\mu}^{1}=\mathcal{A}_{\mu}^{2}, \mathcal{A}_{\mu}^{3}=\phi_{2}=0$, and $\phi_{1}=\sqrt{6} \varphi$ in the Lagrangian given in equation (46) in [20], it matches (4.42), assuming only the Cartan of $\mathrm{SU}(2)$ is excited and setting $\bar{g}=2 \sqrt{2}$.
} 
with $\varphi_{0}$ the value of the scalar at the horizon. The domain wall solution which interpolates between $\mathrm{AdS}_{5}$ and this $\mathrm{AdS}_{2}$ vacuum preserves two real supercharges (enhanced to 4 supercharges in the near horizon limit) and can be thought of as a BPS black hole with a hyperbolic horizon. The black hole entropy is given by

$$
S_{\mathrm{BH}}=\frac{e^{3 g_{0}} \operatorname{vol}\left(M^{3}\right)}{4 G_{N}^{(5)}}=\frac{\operatorname{vol}\left(M^{3}\right)}{\pi} a_{4 d}
$$

It would be interesting to establish this universal relation using field theory methods.

\subsection{4d supergravity}

Here we discuss black hole solutions in four-dimensional gauged supergravity describing the universal twisted compactification of $3 \mathrm{~d} \mathcal{N}=2$ and $\mathcal{N}=4$ SCFTs on a Riemann surface. The field theory setting was briefly discussed in section 3.3 and we refer to [19] for more details. The study of asymptotically $\mathrm{AdS}_{4}$ back holes has received renewed attention, following the discovery of the $3 \mathrm{~d}$ superconformal theories describing the worldvolume of M2-branes and their $\mathrm{AdS}_{4}$ duals [94]; see [95-97] and references thereof. The interpretation of some of these solutions as twisted compactifications of the ABJM theory was provided in $[12,37]$ (see also [98] for earlier work), where the microsocpic entropy of these black holes was reproduced using the supersymmetric index defined in [67]. In this section, we focus on universal solutions describing the twisted compactification of a large class of $3 \mathrm{~d} \mathcal{N}=2$ theories. This was recently used in [19] as a tool to count the black hole microstates for a large class of theories with M-theory as well as massive IIA duals (see also [99, 100] for non-universal examples in massive IIA).

\subsection{1 $\mathcal{N}=2$}

We are interested in asymptotically $\mathrm{AdS}_{4}$ black hole solutions in minimal $\mathcal{N}=2$ gauged supergravity preserving two supercharges. The near-horizon geometry is $\mathrm{AdS}_{2} \times \Sigma_{\mathfrak{g}}$ and the entire solution describes the holographic RG flow from a $3 \mathrm{~d} \mathcal{N}=2$ SCFT on $\Sigma_{\mathfrak{g}}$ to a $1 \mathrm{~d}$ superconformal quantum mechanics. Solutions in non-minimal gauged supergravity were summarized in [12] where references to the extensive earlier literature on the subject can also be found. The minimal theory is obtained by setting $\mathfrak{n}_{a}=\frac{\kappa}{2}, \vec{\phi}=0, L_{a}=1$ in [12]. The Lagrangian reads

$$
e^{-1} \mathcal{L}=R-2 F_{\mu \nu}^{2}-V,
$$

where $V=-12 \bar{g}^{2}$. Comparing to (4.6) we set $\bar{g}=1 / \sqrt{2}$ to normalize $L_{\mathrm{AdS}_{4}}=1$.

The Ansatz of interest takes the form

$$
\begin{aligned}
d s^{2} & =e^{2 f(r)}\left(-d t^{2}+d r^{2}\right)+e^{2 g(r)} d s_{\Sigma_{\mathfrak{g}}}^{2}, \\
F & =-\frac{\kappa}{2 \sqrt{2}} d \operatorname{vol}_{\Sigma_{\mathfrak{g}}},
\end{aligned}
$$

and the BPS equations read

$$
\begin{aligned}
e^{-f} f^{\prime} & =-1+\frac{\kappa}{2} e^{-2 g}, \\
e^{-f} g^{\prime} & =-1-\frac{\kappa}{2} e^{-2 g}
\end{aligned}
$$


For $\kappa=-1$ these equations admit a full analytic solution corresponding to the magnetically charged black hole of [101, 102]. ${ }^{49}$ The uplift of this solution to eleven dimensions, and its interpretation as wrapped M2-branes on a Calabi-Yau five-fold, was given in [98], where other interesting wrapped membranes solutions were also studied. The uplift to massive IIA is provided in [19]. For $\kappa=1$ one finds an IR singularity (see section 3.4 of [98]).

Setting $\kappa=-1$, one finds a regular horizon asymptotic to $\mathrm{AdS}_{2} \times \Sigma_{\mathfrak{g}}$ and the metric functions take the form (4.3) with

$$
e^{f_{0}}=e^{2 g_{0}}=\frac{1}{2}
$$

The entropy of the black hole is given by

$$
S_{\mathrm{BH}}=\frac{e^{2 g_{0}} \operatorname{vol}\left(\Sigma_{\mathfrak{g}}\right)}{4 G_{N}^{(4)}}=(\mathfrak{g}-1) F_{S^{3}}
$$

where in the last equality we used (4.5). This exactly reproduces the large $N$ field theory result (3.40), provided the identification $S_{\mathrm{BH}}=\operatorname{Re} \log Z_{S^{1} \times \Sigma_{\mathfrak{g}}}$, as shown in [19] for this class of solutions. ${ }^{50}$

It is worth highlighting the power behind the rather simple-looking universal relation (4.55). As we have argued on general grounds, this four-dimensional black hole can be uplifted to ten- or eleven-dimensional supergravity in infinitely many ways, depending on the choice of internal six- or seven-manifold; each uplift describes the twisted compactification of a different $3 \mathrm{~d} \mathcal{N}=2 \mathrm{SCFT}$. This fact, combined with the universal relation (4.5), was recently used in [19] to arrive at a microscopic derivation of the entropy of this infinite family of black holes in string and M-theory in the large $N$ limit. Similarly, we expect that the various universal relations derived in this paper will be useful in generalizing these results to even larger classes of black branes in different dimensions.

Non-universal compactifications, i.e., corresponding to turning background flavor fluxes in the SCFT, are described by black holes charged under additional vector multiplets. Such solutions were first considered in [95] and studied further in a number of papers, notably [96, 97] (see [12] for a more complete list of references). The field theory interpretation of these black holes and a microscopic derivation of their entropy was carried out in [12, 37] using the twisted index of [67]. The solution of the minimal theory can be seen as a special case of these, obtained by setting the vector multiplets to zero.

\subsection{2 $\mathcal{N}=4$}

In this section, we investigate which twisted compactifications of $3 \mathrm{~d} \mathcal{N}=4$ SCFTs admit a holographic description. These theories have an $\mathrm{SO}(4) \simeq \mathrm{SU}(2)_{C} \times \mathrm{SU}(2)_{H}$ R-symmetry and the compactification on Riemann surfaces was recently considered in [70]. The appropriate supergravity is minimal $\mathcal{N}=4$ gauged supergravity [103]. This supergravity has sixteen supercharges and bosonic content the graviton, an $\mathrm{SO}(4)$ gauge field, a dilaton, and an axion. It can be obtained as an $S^{7}$ reduction of eleven-dimensional supergravity [104].

\footnotetext{
${ }^{49} \mathrm{~A}$ generalization to include rotation while maintaining supersymmetry was also found in this reference.

${ }^{50}$ This was argued to hold more generally for a larger class of black holes with additional charges in [12].
} 
Following the notation in [104] we denote the two $\mathrm{SU}(2)$ gauge fields by $A$ and $\tilde{A}$, the dilaton by $\phi$, and the axion by $\chi$. Setting $\chi=0$ the bosonic Lagrangian is given by ${ }^{51}$

$$
e^{-1} \mathcal{L}=R * \mathbf{1}-\frac{1}{2} * d \phi \wedge d \phi-\frac{1}{2} e^{-\phi} * F^{I} \wedge F^{I}-\frac{1}{2} e^{\phi} * \tilde{F}^{I} \wedge \tilde{F}^{I}-V * \mathbf{1},
$$

where $I=1,2,3$ are $\mathrm{SU}(2)$ indices and the scalar potential is given by

$$
V=-4 \bar{g}^{2}(2+\cosh \phi) \text {. }
$$

We set $\bar{g}=1 / \sqrt{2}$ in what follows to normalize the scale of the $\mathrm{AdS}_{4}$ vacuum to $L_{\mathrm{AdS}_{4}}=1$.

We are interested in solutions with gauge fields excited only along the Cartan of the two SU(2)'s and thus we consider the following Ansatz:

$$
\begin{aligned}
d s^{2} & =e^{2 f(r)}\left(-d t^{2}+d r^{2}\right)+e^{2 g(r)} d s_{\Sigma_{\mathfrak{g}}}^{2}, \\
F^{I} & =-\frac{\mathfrak{n}}{\sqrt{2}} \delta^{I 3} d \operatorname{vol}\left(\Sigma_{\mathfrak{g}}\right), \quad \tilde{F}^{I}=-\frac{\tilde{\mathfrak{n}}}{\sqrt{2}} \delta^{I 3} d \operatorname{vol}\left(\Sigma_{\mathfrak{g}}\right) .
\end{aligned}
$$

The BPS equations read:

$$
\begin{aligned}
e^{-f} f^{\prime} & =-\frac{1}{2}\left(e^{-\frac{\phi}{2}}+e^{\frac{\phi}{2}}\right)+\frac{e^{-2 g}}{2}\left(\mathfrak{n} e^{\frac{\phi}{2}}+\widetilde{\mathfrak{n}} e^{-\frac{\phi}{2}}\right), \\
e^{-f} g^{\prime} & =-\frac{1}{2}\left(e^{-\frac{\phi}{2}}+e^{\frac{\phi}{2}}\right)-\frac{e^{-2 g}}{2}\left(\mathfrak{n} e^{\frac{\phi}{2}}+\widetilde{\mathfrak{n}} e^{-\frac{\phi}{2}}\right), \\
e^{-f} \phi^{\prime} & =-\left(e^{-\frac{\phi}{2}}-e^{\frac{\phi}{2}}\right)+e^{-2 g}\left(\mathfrak{n} e^{\frac{\phi}{2}}-\widetilde{\mathfrak{n}} e^{-\frac{\phi}{2}}\right), \\
\mathfrak{n}+\widetilde{\mathfrak{n}} & =\kappa .
\end{aligned}
$$

These BPS equations can also be obtained as a truncation of the well studied $\mathrm{U}(1)^{4} \mathrm{STU}$ model of four-dimensional gauge supergravity by setting pairs of the four $\mathrm{U}(1)$ gauge fields equal and two dilatons and all three axions to zero. This was analyzed in some detail in $[12] .^{52}$

To determine the parameter space for which regular black hole solutions exist it suffices to look at possible $\mathrm{AdS}_{2}$ vacua, where the metric functions take the form (4.3). Then, the first two equations in (4.59) imply that $\phi=\phi_{0}$ is a constant and we obtain the set of algebraic equations

$$
\begin{aligned}
2 e^{-f_{0}} & =\left(e^{-\frac{\phi_{0}}{2}}+e^{\frac{\phi_{0}}{2}}\right)-e^{-2 g_{0}}\left(\mathfrak{n} e^{\frac{\phi_{0}}{2}}+\widetilde{\mathfrak{n}} e^{-\frac{\phi_{0}}{2}}\right), \\
0 & =\left(e^{-\frac{\phi_{0}}{2}}+e^{\frac{\phi_{0}}{2}}\right)+e^{-2 g_{0}}\left(\mathfrak{n} e^{\frac{\phi_{0}}{2}}+\widetilde{\mathfrak{n}} e^{-\frac{\phi_{0}}{2}}\right), \\
0 & =\left(e^{-\frac{\phi_{0}}{2}}-e^{\frac{\phi_{0}}{2}}\right)-e^{-2 g_{0}}\left(\mathfrak{n} e^{\frac{\phi_{0}}{2}}-\widetilde{\mathfrak{n}} e^{-\frac{\phi_{0}}{2}}\right) .
\end{aligned}
$$

Note that combining the last two equations it follows that

$$
\mathfrak{n}=\widetilde{\mathfrak{n}}=\frac{\kappa}{2}=-e^{2 g_{0}} .
$$

\footnotetext{
${ }^{51}$ We should note that the axion field $\chi$ is sourced by $F \wedge F$ and thus it is consistent to set it to zero only if $F \wedge F=0$. Since we are interested here in purely magnetic solutions, it is consistent to do so.

${ }^{52}$ To be precise, these match the BPS equations (A.27) in [12], setting $L_{1}=L_{2} \equiv e^{-\frac{\phi}{2}}$ and $L_{3}=L_{4} \equiv e^{\frac{\phi}{2}}$, $\mathfrak{n}_{1}=\mathfrak{n}_{2} \equiv \mathfrak{n}$, and $\mathfrak{n}_{3}=\mathfrak{n}_{4} \equiv \widetilde{\mathfrak{n}}$.
} 
Thus, an $\mathrm{AdS}_{2}$ vacuum is found only for a twist along the diagonal Cartan of $\mathrm{SU}(2)_{C} \times$ $\mathrm{SU}(2)_{H}$. This coincides with the universal black hole solution of the $4 \mathrm{~d} \mathcal{N}=2$ supergravity theory discussed in section 4.4 .1 but now derived as a solution of the $4 \mathrm{~d} \mathcal{N}=4$ minimal supergravity. Indeed, one sees that the solution coincides with the solution (4.54) of the minimally supersymmetric theory. The fact that there are no regular $\mathrm{AdS}_{2}$ vacua preserving four supercharges is consistent with the fact that the topologically twisted index of the corresponding $\mathcal{N}=4$ theory does not have an $N^{3 / 2}$ scaling, as mentioned in section 3.3.

\subsection{Uplifts to $10 \mathrm{~d}$ and $11 \mathrm{~d}$}

As emphasized throughout the paper, a crucial aspect of our story is the fact that the gauged supergravity solutions presented above are universal. This manifests itself in two distinct ways. On the one hand, they are solutions of the minimal gauged supergravity in a given spacetime dimension with a given amount of supersymmetry, i.e., we have switched off any possible matter multiplets. This is the simple holographic dual of the fact that in the SCFT the universal partial topological twists discussed in section 3 involve only the stress-energy tensor multiplet. On the other hand, these minimal supergravity solutions can be embedded into string and M-theory in infinitely many distinct ways by using various consistent truncation results in the literature. This is ultimately the core statement of universality of these constructions. A particular embedding of the domain wall solutions described above into string or M-theory describes the twisted compactification of a particular dual SCFT. Since the relations between IR and UV field theory quantities that follow from these domain wall solutions are insensitive to the particular embedding in higher dimensions, they hold independently of the details of the SCFTs being considered.

Let us briefly describe how these embeddings into ten and eleven-dimensional supergravity are realized. It was shown in [105] and [106] that any supersymmetric $\mathrm{AdS}_{5}$ vacuum of $11 \mathrm{~d}$ or type IIB supergravity admits a consistent truncation to minimal $5 \mathrm{~d} \mathcal{N}=2$ gauged supergravity. The same also holds for any supersymmetric $\mathrm{AdS}_{4}$ solution of $11 \mathrm{~d}$ supergravity which can be consistently truncated to $4 \mathrm{~d}$ minimal $\mathcal{N}=2$ gauged supergravity, as shown in [106]..$^{53}$ Since there are infinitely many such $\mathrm{AdS}_{4}$ and $\mathrm{AdS}_{5}$ solutions we arrive at the conclusion that the universal black string and black hole solutions presented in section 4.3.1 and section 4.4.1 can be embedded in 10 and 11d supergravity, respectively, in infinitely many ways. We note that this does not apply only to the usual Freund-Rubin type solutions and one can also find realizations of the universal flows for warped $\mathrm{AdS}_{5}$ and $\mathrm{AdS}_{4}$ vacua of IIB and 11d supergravity as shown explicitly in [8] and [42], respectively. In [109] and [110] similar consistent truncation results were also derived for the minimal $5 \mathrm{~d} \mathcal{N}=4$ gauged supergravity discussed in section 4.3.2. In [109] it was shown that every supersymmetric $\mathrm{AdS}_{5}$ vacuum of $11 \mathrm{~d}$ supergravity which preserves 16 supercharges admits a consistent truncation to the minimal $5 \mathrm{~d} \mathcal{N}=4$ gauged supergravity. The same holds true for the $\mathrm{AdS}_{5} \times S^{5}$ solution (and its orbifolds preserving with 16 supercharges) in type

\footnotetext{
${ }^{53}$ There is also an embedding of the minimal $4 \mathrm{~d} \mathcal{N}=2$ gauged supergravity in massive IIA supergravity as discussed in [107, 108].
} 
IIB supergravity [110]. ${ }^{54}$ The fact that supersymmetric $\mathrm{AdS}_{7}$ vacua with 16 supercharges of type IIA and 11d supergravity lead to a consistent truncation to the minimal $7 \mathrm{~d} \mathcal{N}=2$ gauged supergravity of section 4.1.1 was shown in [26]. Finally, the solutions of Romans' minimal six-dimensional supergravity theory discussed in section 4.2 can be embedded into massive IIA or type IIB supergraviy using the results of [111] and [112], respectively. All of these consistent truncation results for AdS vacua with 16 supercharges are nicely captured by the recent analysis in [113]. It was shown in [113] that any $\operatorname{AdS}_{D}$ solution of $10 \mathrm{~d}$ or 11d supergravity admits a truncation to the respective minimal (i.e., containing only the gravity multiplet) gauged supergravity in $D$ dimensions. ${ }^{55}$

The upshot of this collection of supergravity results is that every $d$-dimensional SCFT (for $d \geq 3$ ) with a weakly coupled string or M-theory dual captured by a supersymmetric $\mathrm{AdS}_{d+1}$ vacuum of $10 \mathrm{~d}$ or $11 \mathrm{~d}$ supergravity admits a consistent description in terms of minimal gauged supergravity in $d+1$ dimensions. This in turn implies that every such SCFT also enjoys the universal RG flows across dimensions by a twisted compactification on $M_{d-p}$ and the holographic description of this RG flow is in terms of the supergravity domain wall solutions discussed in this section.

This observation is particularly useful in the case of flows between odd-dimensional SCFTs. A simple example of this is the uplift of the $\mathrm{AdS}_{4}$ black hole solution of section 4.4 to eleven-dimensional supergravity and to massive IIA supergravity, recently discussed in [19]. Using the universal relation (4.55) then leads to the microscopic counting of the entropy of a large class of $\mathrm{AdS}_{4}$ black holes [19] both in M-theory and in massive IIA string theory. It would be interesting to apply this approach to the microscopic counting of the entropy of the various black brane solutions described in this work. This requires a detailed understanding of the field theory quantity computing the corresponding entropy, which is currently lacking. One example that might be interesting to study is the $\mathrm{AdS}_{5}$ black hole, with near-horizon geometry (4.47) and entropy (4.50). In analogy to the $\mathrm{AdS}_{4}$ case a natural guess for the field theory quantity that should capture its entropy is the partition function of a $4 \mathrm{~d} \mathcal{N}=2$ theory on $S^{1} \times \mathbb{H}^{3}$, with a partial topological twist on $\mathbb{H}^{3}$. We leave the exploration of this interesting question for future work.

\section{Discussion}

We have established universal relations between physical observables in SCFTs with a continuous R-symmetry, connected by RG flows across dimensions. The precise flows are triggered by a partial topological twist on a compact manifold along the exact UV superconformal R-symmetry. The underlying reason for this universality is the fact that the deformation in the UV amounts to coupling the omnipresent stress-energy tensor multiplet of the SCFT to background fields, namely a background metric and R-symmetry gauge field, and switching off any possible couplings to flavor symmetry currents.

\footnotetext{
${ }^{54}$ We are not aware of any other $\mathrm{AdS}_{5}$ vacua with 16 supercharges in type IIB supergravity. If such solutions exist it is reasonable to conjecture that they will admit a consistent truncation to the $5 \mathrm{~d} \mathcal{N}=4$ minimal supergravity theory.

${ }^{55}$ We note that the results in [113] are somewhat implicit and do not immediately lead to convenient explicit uplift formulas from $D$ to 10 or 11 dimensions.
} 
If the $\mathrm{SCFT}_{d}$ in the UV admits a weakly coupled $\mathrm{AdS}_{d+1}$ gravity dual in string or Mtheory, and if the compactification manifold admits a constant negative-curvature metric, we have provided ample evidence that the $p$-dimensional theory in the IR is also conformal and admits a weakly coupled $\mathrm{AdS}_{p+1}$ dual. The gravitational description provides an explicit realization of the $\mathrm{RG}$ flow across dimensions via a simple domain wall solution of gauged supergravity, interpolating between the $\mathrm{AdS}_{d+1}$ vacuum in the $\mathrm{UV}$ and an $\mathrm{AdS}_{p+1}$ vacuum in the IR. The universality of such flows is understood holographically by the fact that these domain walls can be uplifted to string or M-theory in infinitely many ways.

Our results suggest various interesting questions and directions for future work. Clearly, the most pressing and general question is how to find an independent description of the lowenergy $p$-dimensional SCFTs. We have defined these theories via a twisted compactification of higher-dimensional theories. It would be valuable, however, to have a UV definition in terms of a theory living in the same number of spacetime dimensions. This can be achieved either through a direct definition of the CFT, e.g., by a nonlinear $\sigma$-model on a Ricciflat target manifold in the case $p=2$, or via some $p$-dimensional asymptotically free UV description that flows to the interacting CFT in the IR. The gold standard for this is set by the $\mathcal{N}=1$ and $\mathcal{N}=2$ theories of class $\mathcal{S}$ and the $3 \mathrm{~d}$ theories of class $\mathcal{R}$ arising from M5-branes wrapping Riemann surfaces and three-manifolds, respectively — see [54, 114] and references thereof. Perhaps the most accessible setup to generalize this success to other dimensions is to focus on $d=4, p=2$, in particular the $\alpha$ - and $\beta$-twists of fourdimensional $\mathcal{N}=2$ theories discussed in section 3.2.2 and holographically in section 4.3.2. Some progress in this direction was made recently in $[56,115]$, but there is certainly more to be understood, especially for theories in the large $N$ limit.

When the RG flow is between even-dimensional SCFTs we have relied purely on field theory methods, in particular the power of 't Hooft anomaly matching and superconformal symmetry, to derive exact, finite $N$, relations between quantities in the UV and IR theories. As shown in section 4 these relations are reproduced holographically to leading order in $N$, thus providing strong evidence for the existence of such flows and IR fixed points. The supergravity analysis, however, is not limited to flows between even-dimensional SCFTs and we have used properties of various supergravity solutions to predict similar universal relations when one (or both) of the SCFTs is odd-dimensional, in which case the appropriate physical quantity is the round-sphere free energy. These relations, as currently stated in section 4 , are established only in the large $N$ limit. It would be most interesting to study whether this picture extends beyond the planar limit. Ideally, this could be approached by an exact field theory calculation using supersymmetric localization on the appropriate curved manifold as, e.g., the case of three-dimensional theories on Riemann surfaces [12, 13, 19]. It is likely that this can be generalized further by studying, for instance, suitable supersymmetric partition functions of five-dimensional SCFTs with a partial topological twist on $\Sigma_{\mathfrak{g}}$ to reproduce the holographic prediction in (4.31) by pure field theory methods. Similarly, it should be possible to study four-dimensional $\mathcal{N}=2$ SCFTs with a topological twist on $M_{3}$, with $M_{3}$ an appropriate hyperbolic manifold, to reproduce the black hole entropy in (4.50). An alternative approach to incorporating subleading corrections in $N$ would be to analyze the universal RG flows holographically, including higher-curvature 
corrections in gauged supergravity. Although a technically challenging problem in general, this was addressed successfully in [116] for various domain walls interpolating between AdS vacua corresponding to RG flows between even-dimensional SCFTs. It would be very interesting to extend this approach to the various domain wall solutions described here. It would also be interesting to study whether there are universal relations among other physical observables in the $p$-dimensional and $d$-dimensional SCFTs, e.g., Wilson loop expectation values or partition functions with other insertions.

A series of interesting questions relate to the choice of $M_{d-p}$. As discussed, most universal RG flows we have studied, both in field theory as well as holographically, require $M_{d-p}$ to be hyperbolic or negatively curved in order for the IR $p$-dimensional theory to be unitary. ${ }^{56}$ We do not have an explanation why this must be the case in general and it would be interesting to have a better understanding of this. In addition, the supergravity solutions we constructed require the metric on the compact manifold $M_{d-p}$ in the IR to be Einstein. From field theory considerations, however, it is clear that in the UV one should be able to use any metric on $M_{d-p}$, since we are performing a topological twist. Thus, holography suggests that the RG flow across dimensions uniformizes the metric on $M_{d-p}$. This has been understood in some detail for Riemann surfaces in [117] and it would certainly be very interesting to explore the interplay between holographic RG flows and uniformization for higher-dimensional manifolds. The Einstein metric at the IR end of the RG flow may still admit a moduli space of deformations compatible with the Einstein condition. These moduli should correspond to exactly marginal couplings in the $p$-dimensional SCFT. This is also clear from the gravitational construction of the holographic dual, where the moduli of the Einstein metric on $M_{d-p}$ lead to massless scalar excitations on the $\mathrm{AdS}_{p+1}$ space dual to the IR SCFT. This picture is well established for the case $d=6, p=4[4,5,39,114]$ and partially explored for the case $d=6, p=2$ in [118]. Additional exactly marginal deformations of the $p$-dimensional SCFT may be present if the $d$-dimensional parent theory admits global, non-R, symmetries for which one can turn on flat connections on $M_{d-p}$. Finally, let us note that we have assumed throughout the paper that $M_{d-p}$ is smooth and compact. It is natural to consider generalizations of this setup to allow for boundaries, punctures, or other defects. It would be interesting to study whether there is a generalization of the universal relations uncovered in this work in these more general situations.

As discussed at length above, the main reason behind the existence of the universal $\mathrm{RG}$ flows across dimensions is that the partial topological twist triggering the RG flow is performed using only background fields that couple to the universal stress-energy tensor multiplet, which exists for all SCFTs with a continuous R-symmetry. The holographic manifestation of this universality is realized by the fact that gauged supergravities always admit a truncation to a universal sector including only the gravity multiplet. In addition, these $(d+1)$-dimensional "minimal" supergravities arise as universal consistent truncations from string and M-theory in infinitely many ways, that are distinguished by the choice of internal manifold (and the fluxes through it) used for the reduction from 10 (or 11)

\footnotetext{
${ }^{56} \mathrm{An}$ exception to this general rule is the case of $6 \mathrm{~d}$ SCFTs on a 5-manifold at large $N$, as noted in section 4.1.2. We have assumed throughout the paper that the UV $d$-dimensional theory is unitary.
} 
dimensions. This holographic perspective suggests that SCFTs with a holographic dual enjoy a truncation of the OPE, at least at large $N$, for operators belonging to the stressenergy multiplet. It would be very interesting to understand the mechanism behind such an OPE truncation, as this could offer an explanation and organizational principle for the plethora of consistent truncations in the supergravity literature.

A few other observations made throughout this work deserve further analysis. What is the relation between unitarity of the IR $p$-dimensional theory at the end of the RG flow across dimensions and the Hofman-Maldacena-type bounds for the parent $d$-dimensional UV theory? Is there a Hofman-Maldacena-type bound on the four anomaly coefficients in six-dimensional SCFTs? ${ }^{57}$

From all the examples of RG flows across dimensions studied here, it seems that to obtain an SCFT with an AdS dual in the IR, the UV theory must also be conformal and strongly interacting. We are not aware of any a priori reason for this to be the case, and it would be interesting to find more general examples of RG flows across dimensions, where the UV theory is not strongly interacting. In appendix B we observe intriguing relations between field theory observables such as conformal anomalies and free energies and the entropies of various supersymmetric black branes. It is desirable to put these on a firmer footing and calculate the black brane entropies from a more rigorous field theory setting, as done recently for supersymmetric black holes in $\operatorname{AdS}_{4}[12,19]$. Finally, it is natural to wonder whether there is some notion of a "monotonicity theorem", similar to the $c_{-}, a_{-}$, or $F$-theorems for RG flows across dimensions. Based on the universal relations between conformal anomalies and free energies studied in this work it is clear that simply comparing the natural monotonic quantity in the IR $p$-dimensional SCFT with the one in the UV $d$-dimensional SCFT is too naive. Perhaps one should search for a more refined definition of a monotonic function along the RG flow which removes the explicit factor of the volume of the compactification manifold $M_{d-p}$.

It is clear that the universal RG flows described here provide a fertile area for exploring the physics of supersymmetric QFTs and holography. We expect many further exciting developments ahead of us.

\section{Acknowledgments}

We are grateful to Fabio Apruzzi, Marco Baggio, Chris Beem, Davide Cassani, Eoin Ó Colgáin, Antón Faedo, Friðrik Gautason, Diego Hofman, Edoardo Lauria, Vincent Min, Krzysztof Pilch, Alberto Zaffaroni, and especially Francesco Benini for interesting discussions. The work of NB is supported in part by the starting grant BOF/STG/14/032 from KU Leuven, by an Odysseus grant G0F9516N from the FWO, and by the KU Leuven C1 grant ZKD1118 C16/16/005. In addition NB acknowledges support by the Belgian Federal Science Policy Office through the Inter-University Attraction Pole P7/37, and by the COST Action MP1210 The String Theory Universe. PMC is supported by Nederlandse Organisatie voor Wetenschappelijk Onderzoek (NWO) via a Vidi grant. The work of PMC is part of the Delta ITP consortium, a program of the NWO that is funded by the Dutch

\footnotetext{
${ }^{57}$ See for example $[35,77,119]$ for some recent results and conjectures in this direction.
} 
Ministry of Education, Culture and Science (OCW). PMC would like to thank the ITP at Stanford University and KU Leuven for kind hospitality while part of this work was carried out. Both of us are grateful for the warm hospitality offered by the CERN Theory Group at the final stages of this work.

\section{A Conventions and normalizations}

In this appendix we review our conventions and normalizations and collect useful formulae used throughout the paper.

\section{A.1 Characteristic classes}

The total Chern class of a vector bundle and the Pontryagin class of the tangent bundle are given by (here we are following the conventions in [28], see also [120])

$$
\begin{aligned}
& C(\mathcal{F})=\operatorname{det}\left(1+i \frac{\mathcal{F}}{2 \pi}\right)=1+c_{1}(\mathcal{F})+c_{2}(\mathcal{F})+\cdots, \\
& P(\mathcal{T})=\operatorname{det}\left(1-\frac{\mathcal{R}}{2 \pi}\right)=1+p_{1}(\mathcal{T})+p_{2}(\mathcal{T})+\cdots,
\end{aligned}
$$

with $\mathcal{F}$ the field strength two-form, which we take to be antihermitian, and $\mathcal{R}$ the Riemann curvature two-form. From this we find the following expressions for the first two Chern and Pontryagin classes

$$
\begin{array}{rlrl}
c_{1}(\mathcal{F}) & =\frac{i}{2 \pi} \operatorname{Tr} \mathcal{F}, & c_{2}(\mathcal{F})=\frac{1}{(2 \pi)^{2}} \frac{1}{2}\left(\operatorname{Tr} \mathcal{F}^{2}-(\operatorname{Tr} \mathcal{F})^{2}\right), \\
p_{1}(\mathcal{T})=-\frac{1}{(2 \pi)^{2}} \frac{1}{2} \operatorname{Tr} \mathcal{R}^{2}, & p_{2}(\mathcal{T})=\frac{1}{(2 \pi)^{4}}\left(\frac{1}{8}\left(\operatorname{Tr} \mathcal{R}^{2}\right)^{2}-\frac{1}{4} \operatorname{Tr} \mathcal{R}^{4}\right),
\end{array}
$$

where in the first line the Tr is over "gauge" indices in the fundamental representation and in the second line the $\operatorname{Tr}$ is over tangent frame indices. Note that $p_{1}(\mathcal{T})$ differs by a sign from that used in [33] which explains the minus sign difference in $\beta$ in (2.3) in comparison to the expression in [33].

A useful property of characteristic classes is the splitting principle. The total Chern and Pontryagin classes $C(U)=\sum_{i} c_{i}(U), P(E)=\sum_{i} p_{i}(E)$, decompose under the direct sum of vector bundles as

$$
C(U \oplus V)=C(U) C(V), \quad P(E \oplus F)=P(E) P(F) .
$$

In particular, it follows from the second relation that the first and second Pontryagin classes satisfy

$$
p_{1}(E \oplus F)=p_{1}(E)+p_{1}(F), \quad p_{2}(E \oplus F)=p_{2}(E)+p_{2}(F)+p_{1}(E) p_{1}(F),
$$

and similarly for Chern classes. Given a decomposition of the tangent bundle as a sum of complex line bundles with first Chern classes $e_{i}$ one has:

$$
p_{1}(\mathcal{T})=\sum_{i} e_{i}^{2}, \quad p_{2}(\mathcal{T})=\sum_{i<j} e_{i}^{2} e_{j}^{2}
$$


Finally we note that for a four-manifold which is a product of two Riemann surfaces $\Sigma_{1} \times \Sigma_{2}$, the integrated first Pontryagin class, $P_{1}$, and the Euler characteristic, $\chi$, are $P_{1}=0$ and $\chi=4\left(\mathfrak{g}_{1}-1\right)\left(\mathfrak{g}_{2}-1\right)$.

\section{A.2 Metric on Riemann surfaces}

Throughout this paper we often consider smooth Riemann surfaces $\Sigma_{\mathfrak{g}}$ of genus $\mathfrak{g}$. We always put a constant curvature metric on these manifolds with the following explicit form

$$
d s_{\Sigma_{\mathfrak{g}}}^{2}=e^{2 h\left(x_{1}, x_{2}\right)}\left(d x_{1}^{2}+d x_{2}^{2}\right), \quad h\left(x_{1}, x_{2}\right)= \begin{cases}-\log \frac{1+x_{1}^{2}+x_{2}^{2}}{2} & \text { for } \mathfrak{g}=0 \\ \frac{1}{2} \log 2 \pi & \text { for } \mathfrak{g}=1 \\ -\log x_{2} & \text { for } \mathfrak{g}>1\end{cases}
$$

The volume form $d \operatorname{vol}_{\Sigma_{\mathfrak{g}}} \equiv e^{2 h} d x_{1} \wedge d x_{2}$ integrates to:

$$
\int d \operatorname{vol}\left(\Sigma_{\mathfrak{g}}\right)=2 \pi \eta_{\Sigma}, \quad \eta_{\Sigma}=\left\{\begin{array}{ll}
2|\mathfrak{g}-1| & \text { for } \mathfrak{g} \neq 1 \\
1 & \text { for } \mathfrak{g}=1
\end{array} .\right.
$$

The normalized curvature of $\Sigma_{\mathfrak{g}}$ is denoted by $\kappa=\{1,0,-1\}$ for $\mathfrak{g}=0, \mathfrak{g}=1$, and $\mathfrak{g}>1$, respectively. We note that with these definitions and using (A.7) one has the relation $\kappa \eta_{\Sigma}=-2(\mathfrak{g}-1)$ for all $\mathfrak{g}$. Finally, $t_{\mathfrak{g}}$ denotes the first Chern class of the tangent bundle of $\Sigma_{\mathfrak{g}}$, which in our normalizations integrates to $\int_{\Sigma_{\mathfrak{g}}} t_{\mathfrak{g}}=\eta_{\Sigma}$.

\section{B Entropy of black branes}

All regular supergravity solutions discussed in this paper can be viewed as extremal $(p-1)$ brane solutions in $d+1$ spacetime dimensions. The near horizon geometry is of the form:

$$
d s_{d+1}^{2}=e^{2 f_{0}} d s_{\mathrm{AdS}_{p+1}}^{2}+e^{2 g_{0}} d s_{M_{d-p}}^{2},
$$

where

$$
d s_{\mathrm{AdS}_{p+1}}^{2}=\frac{1}{r^{2}}\left(-d t^{2}+d r^{2}+d z_{1}^{2}+\cdots+d z_{p-1}^{2}\right),
$$

and $d s_{M_{d-p}}^{2}$ is the metric on the compact horizon of the $(p-1)$-brane. The field theory interpretation of these $(p-1)$-brane solutions is given by the universal RG flows across dimensions discussed extensively in the main text. In particular, there is a $p$-dimensional SCFT captured holographically by the $\mathrm{AdS}_{p+1}$ factor in the near horizon geometry. This is the IR SCFT which arises from a $d$-dimensional UV SCFT via the RG flow across dimensions. For $p$ even, the conformal anomaly coefficients of the SCFT can be computed holographically using (4.4). Another physically interesting quantity is the entropy density of the black brane. To compute this we take the spatial coordinates on the boundary of $\operatorname{AdS}_{p+1}, z_{i}, i=1, \cdots, p-1$, to have a finite range $z_{i} \in\left[0, l_{i}\right]$. We can then easily compute the Bekenstein-Hawking entropy per unit of spatial volume, $V \equiv l_{1} \times \ldots \times l_{p-1},{ }^{58}$

$$
s \equiv \frac{S}{V}=\frac{\operatorname{vol}\left(\tilde{M}_{d-p}\right) e^{(p-1) f_{0}}}{4 G_{N}^{(d+1)}}=\frac{e^{(p-1) f_{0}}}{4 G_{N}^{(p+1)}},
$$

\footnotetext{
${ }^{58}$ We note that this is the spatial volume of the boundary of $\operatorname{AdS}_{p+1}$ and not that of the horizon $M_{d-p}$.
} 
where $d s_{\tilde{M}_{d-p}}^{2} \equiv e^{2 g_{0}} d s_{M_{d-p}}^{2}$ and in the last equality we used the relation between the Newton constants in $p+1$ and $d+1$ dimensions,

$$
\frac{1}{G_{N}^{(d+1)}}=\frac{\operatorname{vol}\left(\tilde{M}_{d-p}\right)}{G_{N}^{(p+1)}} .
$$

Combining this with the formulae (4.4) for the central charges in a $p$-dimensional SCFT for $p=2,4,6$ we can write the entropy density of a $(p-1)$-brane in terms of the corresponding Weyl anomaly coefficient:

$$
\begin{aligned}
& s(D 1)=\frac{1}{6} c_{2 d}, \\
& s(D 3)=\frac{2}{\pi} a_{4 d}, \\
& s(D 5)=\frac{7}{12 \pi^{2}} a_{6 d} .
\end{aligned}
$$

In the first line we defined $c_{2 d} \equiv c_{r}=c_{l}$, which is valid in the large $N$ limit.

Similarly, for $p=3,5$ we can write the entropy density in terms of the corresponding round-sphere free energy in (4.5) for the $\mathrm{SCFT}_{p}$, in the following way

$$
\begin{aligned}
& s(D 2)=\frac{1}{2 \pi} F_{S^{3}}, \\
& s(D 4)=-\frac{3}{4 \pi^{2}} F_{S^{5}} .
\end{aligned}
$$

We emphasize that the anomaly coefficients and free energies appearing in the entropy densities (B.5)-(B.9) are those corresponding to the $p$-dimensional SCFT living on the worldvolume of the $(p-1)$-brane, i.e., IR quantities. The universal relations that we have established in this work, however, allow us to relate these to UV quantities of the $d$-dimensional SCFT, dual to the asymptotically locally $\mathrm{AdS}_{d+1}$ boundary.

Finally, we point out that the case $p=1$, i.e., an $\mathrm{AdS}_{2}$ near horizon region, is somewhat special since we do not have a microscopically well established $\mathrm{AdS}_{2} / \mathrm{CFT}_{1}$ duality. The best understood setup is that of extremal black holes in $\mathrm{AdS}_{4}$ discussed in section 4.4.

\section{Universal RG flows in the same dimension}

While the main focus in this work has been to uncover universal relations between SCFTs connected by an RG flow across dimensions, it is important to note that there are also similar relations for SCFTs living in the same number of spacetime dimensions. A particularly simple relation between the conformal anomalies of $4 \mathrm{~d} \mathcal{N}=2$ and $\mathcal{N}=1$ SCFTs connected by a specific universal RG flow was derived by Tachikawa and Wecht in [25]. Inspired by this here we study the possibility of a similar result for $2 \mathrm{~d}$ SCFTs.

Let us first revisit the main result in [25] from the perspective of our discussion. A particular class of examples of $4 \mathrm{~d} \mathcal{N}=2$ and $\mathcal{N}=1$ SCFTs which are connected by the RG flow of [25] are the MN $\mathcal{N}=2$ and $M N \mathcal{N}=1$ SCFTs discussed in section 3.1.2 above. The relation between the anomalies of these two classes of $4 \mathrm{~d}$ SCFTs can be obtained by 
using the two universal relations, (3.13) and (3.15), of these anomalies to the ones of the class of $\mathcal{N}=(2,0)$ SCFTs in 6d. Using (3.13) and inverting the matrix in (3.15) we find that the $4 \mathrm{~d} \mathcal{N}=2$ and $\mathcal{N}=1$ central charges are related by

$$
\left(\begin{array}{l}
a_{4 d} \\
c_{4 d}
\end{array}\right)_{\mathcal{N}=1}=\frac{3}{32}\left(\begin{array}{cc}
12 & -3 \\
-4 & 13
\end{array}\right)\left(\begin{array}{l}
a_{4 d} \\
c_{4 d}
\end{array}\right)_{\mathcal{N}=2} .
$$

This is precisely the relation derived in [25].

We now ask whether the various theories obtained in $2 \mathrm{~d}$ by twisted compactification of $4 \mathrm{~d}$ theories discussed in the main text could be connected by similar two-dimensional RG flows. Consider the theories obtained by $\alpha$ - and $\beta$-twists discussed in section 3.2.2 and the universal $(0,2)$ twist of section 3.2.1. Since for the $\alpha$-twist the matrix relating the $2 \mathrm{~d}$ and $4 \mathrm{~d}$ anomaly coefficients is not invertible we cannot apply the same procedure as above. For the $\beta$-twist, however, the matrix in (3.35) is invertible and one can combine that with the matrix in (3.24) to find the following putative analog of the Tachikawa-Wecht relation in $2 \mathrm{~d}$ :

$$
\left(\begin{array}{l}
c_{r} \\
c_{l}
\end{array}\right)_{(0,2)}=\frac{1}{9}\left(\begin{array}{cc}
5 & -1 \\
2 & 2
\end{array}\right)\left(\begin{array}{l}
c_{r} \\
c_{l}
\end{array}\right)_{(0,4)} .
$$

The existence of a two-dimensional RG flow realizing (C.2), however, seems to be in conflict with 't Hooft anomaly matching of the gravitational anomaly $k$ which requires $\left(c_{r}-c_{l}\right)_{\mathrm{IR}}=$ $\left(c_{r}-c_{l}\right)_{\mathrm{UV}}$, whereas (C.2) leads to $\left(c_{r}-c_{l}\right)_{(0,2)}=\frac{1}{3}\left(c_{r}-c_{l}\right)_{(0,4)}$. Thus, one naively concludes that such a flow can exist only for theories with $c_{l}=c_{r}$.

We also observe that, as discussed in section 3.2.3, for $4 \mathrm{~d} \mathcal{N}=3$ theories one can have universal flows to $2 \mathrm{~d}$ preserving $\mathcal{N}=(0,6)$ and $\mathcal{N}=(2,4)$ supersymmetry. Comparing the central charges (3.37) and (3.38) to those obtained for the universal $\mathcal{N}=(0,2)$ twist $(3.24)$, and using that $a_{4 d}=c_{4 d}$ for $4 \mathrm{~d} \mathcal{N}=3$ SCFTs, one obtains the relations

$$
c_{(0,2)}=\frac{5}{9} c_{(0,6)}, \quad c_{(0,2)}=\frac{4}{9} c_{(2,4)} .
$$

Here we have denoted $c_{r}=c_{l} \equiv c$, which holds automatically for these $2 \mathrm{~d}$ theories. The second equation above is a special case of the relation in (C.2), applied to $\mathcal{N}=(0,4)$ theories with $c_{r}=c_{l}$. We also note that the $\beta$-twist, applied to $\mathcal{N}=4 \mathrm{SYM}$ leads to a $2 \mathrm{~d}$ theory with enhanced $\mathcal{N}=(4,4)$ and central charge $c_{(4,4)}=6(\mathfrak{g}-1) d_{G}$, with $d_{G}$ the dimension of the gauge group, while the universal $(0,2)$ twist applied to the $\mathcal{N}=4$ theory leads to a $2 \mathrm{~d}$ SCFT with central charge $c_{(0,2)}=\frac{8}{3}(\mathfrak{g}-1) d_{G}$, consistent with the second equation in (C.3). See [7] for a detailed discussion of partial topological twists of $\mathcal{N}=4 \mathrm{SYM}$.

We emphasize that although these relations among $2 \mathrm{~d}$ central charges are suggestive, we have not derived them by studying concrete two-dimensional RG flows realized in a specific SCFT. It would be most interesting to explore this further and understand whether such universal two-dimensional RG flows exist. 
Open Access. This article is distributed under the terms of the Creative Commons Attribution License (CC-BY 4.0), which permits any use, distribution and reproduction in any medium, provided the original author(s) and source are credited.

\section{References}

[1] E. Witten, Topological Quantum Field Theory, Commun. Math. Phys. 117 (1988) 353 [INSPIRE].

[2] G. Festuccia and N. Seiberg, Rigid Supersymmetric Theories in Curved Superspace, JHEP 06 (2011) 114 [arXiv:1105.0689] [INSPIRE].

[3] J. Teschner, Exact Results on $\mathcal{N}=2$ Supersymmetric Gauge Theories, in New Dualities of Supersymmetric Gauge Theories, J. Teschner ed., Springer (2016), pp. 1-30, [arXiv: 1412.7145].

[4] I. Bah, C. Beem, N. Bobev and B. Wecht, AdS/CFT Dual Pairs from M5-Branes on Riemann Surfaces, Phys. Rev. D 85 (2012) 121901 [arXiv:1112.5487] [InSPIRE].

[5] I. Bah, C. Beem, N. Bobev and B. Wecht, Four-Dimensional SCFTs from M5-Branes, JHEP 06 (2012) 005 [arXiv: 1203.0303] [INSPIRE].

[6] F. Benini and N. Bobev, Exact two-dimensional superconformal R-symmetry and c-extremization, Phys. Rev. Lett. 110 (2013) 061601 [arXiv:1211.4030] [inSPIRE].

[7] F. Benini and N. Bobev, Two-dimensional SCFTs from wrapped branes and c-extremization, JHEP 06 (2013) 005 [arXiv: 1302.4451] [INSPIRE].

[8] N. Bobev, K. Pilch and O. Vasilakis, (0,2) SCFTs from the Leigh-Strassler fixed point, JHEP 06 (2014) 094 [arXiv: 1403.7131] [INSPIRE].

[9] F. Benini, N. Bobev and P.M. Crichigno, Two-dimensional SCFTs from D3-branes, JHEP 07 (2016) 020 [arXiv: 1511.09462] [InSPIRE].

[10] D. Kutasov and J. Lin, (0,2) Dynamics From Four Dimensions, Phys. Rev. D 89 (2014) 085025 [arXiv: 1310.6032] [INSPIRE].

[11] D. Kutasov and J. Lin, (0,2) ADE Models From Four Dimensions, arXiv:1401.5558 [INSPIRE].

[12] F. Benini, K. Hristov and A. Zaffaroni, Black hole microstates in AdS 4 from supersymmetric localization, JHEP 05 (2016) 054 [arXiv: 1511.04085] [INSPIRE].

[13] S.M. Hosseini and A. Zaffaroni, Large- $N$ matrix models for $3 d \mathcal{N}=2$ theories: twisted index, free energy and black holes, JHEP 08 (2016) 064 [arXiv: 1604.03122] [INSPIRE].

[14] S.M. Hosseini and N. Mekareeya, Large $N$ topologically twisted index: necklace quivers, dualities and Sasaki-Einstein spaces, JHEP 08 (2016) 089 [arXiv:1604.03397] [INSPIRE].

[15] A. Amariti, L. Cassia and S. Penati, c-extremization from toric geometry, arXiv:1706.07752 [INSPIRE].

[16] K.A. Intriligator and B. Wecht, The exact superconformal $R$ symmetry maximizes a, Nucl. Phys. B 667 (2003) 183 [hep-th/0304128] [INSPIRE].

[17] D.L. Jafferis, The Exact Superconformal R-Symmetry Extremizes Z, JHEP 05 (2012) 159 [arXiv: 1012.3210] [INSPIRE]. 
[18] D.L. Jafferis, I.R. Klebanov, S.S. Pufu and B.R. Safdi, Towards the F-Theorem: $N=2$ Field Theories on the Three-Sphere, JHEP 06 (2011) 102 [arXiv:1103.1181] [INSPIRE].

[19] F. Azzurli, N. Bobev, P.M. Crichigno, V.S. Min and A. Zaffaroni, A Universal Counting of Black Hole Microstates in AdS $S_{4}$, arXiv: 1707.04257 [INSPIRE].

[20] J.M. Maldacena and C. Nunez, Supergravity description of field theories on curved manifolds and a no go theorem, Int. J. Mod. Phys. A16 (2001) 822 [hep-th/0007018].

[21] M. Bershadsky, C. Vafa and V. Sadov, D-branes and topological field theories, Nucl. Phys. B 463 (1996) 420 [hep-th/9511222] [INSPIRE].

[22] J.P. Gauntlett, Branes, calibrations and supergravity, hep-th/0305074 [INSPIRE].

[23] R. Corrado, M. Günaydin, N.P. Warner and M. Zagermann, Orbifolds and flows from gauged supergravity, Phys. Rev. D 65 (2002) 125024 [hep-th/0203057] [inSPIRE].

[24] R. Corrado and N. Halmagyi, $N=1$ field theories and fluxes in IIB string theory, Phys. Rev. D 71 (2005) 046001 [hep-th/0401141] [INSPIRE].

[25] Y. Tachikawa and B. Wecht, Explanation of the Central Charge Ratio 27/32 in Four-Dimensional Renormalization Group Flows between Superconformal Theories, Phys. Rev. Lett. 103 (2009) 061601 [arXiv:0906. 0965] [INSPIRE].

[26] A. Passias, A. Rota and A. Tomasiello, Universal consistent truncation for $6 d / 7 d$ gauge/gravity duals, JHEP 10 (2015) 187 [arXiv:1506.05462] [INSPIRE].

[27] F. Apruzzi, M. Fazzi, A. Passias, A. Rota and A. Tomasiello, Six-Dimensional Superconformal Theories and their Compactifications from Type IIA Supergravity, Phys. Rev. Lett. 115 (2015) 061601 [arXiv:1502.06616] [inSPIRE].

[28] J.A. Harvey, TASI 2003 lectures on anomalies, hep-th/0509097 [INSPIRE].

[29] W. Boucher, D. Friedan and A. Kent, Determinant Formulae and Unitarity for the $N=2$ Superconformal Algebras in Two-Dimensions or Exact Results on String Compactification, Phys. Lett. B 172 (1986) 316 [INSPIRE].

[30] D. Anselmi, D.Z. Freedman, M.T. Grisaru and A.A. Johansen, Nonperturbative formulas for central functions of supersymmetric gauge theories, Nucl. Phys. B 526 (1998) 543 [hep-th/9708042] [INSPIRE].

[31] F. Bastianelli, S. Frolov and A.A. Tseytlin, Conformal anomaly of $(2,0)$ tensor multiplet in six-dimensions and AdS/CFT correspondence, JHEP 02 (2000) 013 [hep-th/0001041] [INSPIRE].

[32] M. Kulaxizi and A. Parnachev, Supersymmetry Constraints in Holographic Gravities, Phys. Rev. D 82 (2010) 066001 [arXiv: 0912.4244] [INSPIRE].

[33] C. Cordova, T.T. Dumitrescu and K. Intriligator, Anomalies, renormalization group flows and the a-theorem in six-dimensional $(1,0)$ theories, JHEP 10 (2016) 080 [arXiv: 1506. 03807] [INSPIRE].

[34] M. Beccaria and A.A. Tseytlin, Conformal anomaly c-coefficients of superconformal $6 d$ theories, JHEP 01 (2016) 001 [arXiv:1510.02685] [InSPIRE].

[35] S. Yankielowicz and Y. Zhou, Supersymmetric Rényi entropy and Anomalies in 6d $(1,0)$ SCFTs, JHEP 04 (2017) 128 [arXiv: 1702.03518] [INSPIRE].

[36] M. Beccaria and A.A. Tseytlin, $C_{T}$ for higher derivative conformal fields and anomalies of $(1,0)$ superconformal $6 d$ theories, JHEP 06 (2017) 002 [arXiv: 1705. 00305] [INSPIRE]. 
[37] F. Benini, K. Hristov and A. Zaffaroni, Exact microstate counting for dyonic black holes in $A d S_{4}$, Phys. Lett. B 771 (2017) 462 [arXiv: 1608.07294] [inSPIRE].

[38] L.F. Alday, F. Benini and Y. Tachikawa, Liouville/Toda central charges from M5-branes, Phys. Rev. Lett. 105 (2010) 141601 [arXiv:0909.4776] [inSPIRE].

[39] F. Benini, Y. Tachikawa and B. Wecht, Sicilian gauge theories and $N=1$ dualities, JHEP 01 (2010) 088 [arXiv:0909.1327] [INSPIRE].

[40] G. 't Hooft, Naturalness, chiral symmetry, and spontaneous chiral symmetry breaking, NATO Sci. Ser. B 59 (1980) 135 [inSPIRE].

[41] S. Giombi and I.R. Klebanov, Interpolating between a and F, JHEP 03 (2015) 117 [arXiv: 1409.1937] [INSPIRE].

[42] N. Bobev, V.S. Min and K. Pilch, Black Holes in AdS 4 from Mass-deformed M2-branes, to appear.

[43] W. Nahm, Supersymmetries and their Representations, Nucl. Phys. B 135 (1978) 149 [INSPIRE].

[44] K. Ohmori, H. Shimizu, Y. Tachikawa and K. Yonekura, Anomaly polynomial of general $6 d$ SCFTs, PTEP 2014 (2014) 103B07 [arXiv: 1408.5572] [inSPIRE].

[45] K. Intriligator, $6 d, \mathcal{N}=(1,0)$ Coulomb branch anomaly matching, JHEP 10 (2014) 162 [arXiv: 1408.6745] [INSPIRE].

[46] S.S. Razamat, C. Vafa and G. Zafrir, $4 d \mathcal{N}=1$ from $6 d(1,0)$, JHEP 04 (2017) 064 [arXiv: 1610.09178] [INSPIRE].

[47] J.P. Gauntlett, N. Kim and D. Waldram, M Five-branes wrapped on supersymmetric cycles, Phys. Rev. D 63 (2001) 126001 [hep-th/0012195] [INSPIRE].

[48] F. Apruzzi, F. Hassler, J.J. Heckman and I.V. Melnikov, From 6D SCFTs to Dynamic GLSMs, Phys. Rev. D 96 (2017) 066015 [arXiv:1610.00718] [inSPIRE].

[49] E. Witten, Solutions of four-dimensional field theories via M-theory, Nucl. Phys. B 500 (1997) 3 [hep-th/9703166] [INSPIRE].

[50] D. Gaiotto and J. Maldacena, The gravity duals of $N=2$ superconformal field theories, JHEP 10 (2012) 189 [arXiv:0904.4466] [InSPIRE].

[51] M. Bershadsky, A. Johansen, V. Sadov and C. Vafa, Topological reduction of $4-D S Y M$ to 2 - D $\sigma$-models, Nucl. Phys. B 448 (1995) 166 [hep-th/9501096] [inSPIRE].

[52] J.P. Gauntlett and N. Kim, M five-branes wrapped on supersymmetric cycles. 2., Phys. Rev. D 65 (2002) 086003 [hep-th/0109039] [INSPIRE].

[53] B.S. Acharya, J.P. Gauntlett and N. Kim, Five-branes wrapped on associative three cycles, Phys. Rev. D 63 (2001) 106003 [hep-th/0011190] [inSPIRE].

[54] T. Dimofte, D. Gaiotto and S. Gukov, Gauge Theories Labelled by Three-Manifolds, Commun. Math. Phys. 325 (2014) 367 [arXiv:1108.4389] [inSPIRE].

[55] A. Kapustin, Holomorphic reduction of $N=2$ gauge theories, Wilson-' $t$ Hooft operators and S-duality, hep-th/0612119 [INSPIRE].

[56] P. Putrov, J. Song and W. Yan, (0,4) dualities, JHEP 03 (2016) 185 [arXiv:1505.07110] [INSPIRE]. 
[57] A.D. Shapere and Y. Tachikawa, Central charges of $N=2$ superconformal field theories in four dimensions, JHEP 09 (2008) 109 [arXiv:0804.1957] [INSPIRE].

[58] C. Beem, M. Lemos, P. Liendo, W. Peelaers, L. Rastelli and B.C. van Rees, Infinite Chiral Symmetry in Four Dimensions, Commun. Math. Phys. 336 (2015) 1359 [arXiv:1312.5344] [INSPIRE].

[59] S. Cecotti, J. Song, C. Vafa and W. Yan, Superconformal Index, BPS Monodromy and Chiral Algebras, JHEP 11 (2017) 013 [arXiv: 1511.01516] [INSPIRE].

[60] A. Schwimmer and N. Seiberg, Comments on the $N=2, N=3, N=4$ Superconformal Algebras in Two-Dimensions, Phys. Lett. B 184 (1987) 191 [inSPIRE].

[61] C. Beem, M. Lemos, P. Liendo, L. Rastelli and B.C. van Rees, The $\mathcal{N}=2$ superconformal bootstrap, JHEP 03 (2016) 183 [arXiv: 1412 .7541] [INSPIRE].

[62] O. Aharony and M. Evtikhiev, On four dimensional $N=3$ superconformal theories, JHEP 04 (2016) 040 [arXiv: 1512.03524] [INSPIRE].

[63] I. García-Etxebarria and D. Regalado, $\mathcal{N}=3$ four dimensional field theories, JHEP 03 (2016) 083 [arXiv: 1512.06434] [INSPIRE].

[64] O. Aharony and Y. Tachikawa, S-folds and $4 d N=3$ superconformal field theories, JHEP 06 (2016) 044 [arXiv: 1602.08638] [InSPIRE].

[65] A. Amariti, L. Cassia and S. Penati, Surveying 4d SCFTs twisted on Riemann surfaces, JHEP 06 (2017) 056 [arXiv:1703.08201] [InSPIRE].

[66] A. Kapustin, B. Willett and I. Yaakov, Exact Results for Wilson Loops in Superconformal Chern-Simons Theories with Matter, JHEP 03 (2010) 089 [arXiv:0909.4559] [INSPIRE].

[67] F. Benini and A. Zaffaroni, A topologically twisted index for three-dimensional supersymmetric theories, JHEP 07 (2015) 127 [arXiv: 1504.03698] [INSPIRE].

[68] F. Benini and A. Zaffaroni, Supersymmetric partition functions on Riemann surfaces, Proc. Symp. Pure Math. 96 (2017) 13 [arXiv: 1605. 06120] [INSPIRE].

[69] C. Closset and H. Kim, Comments on twisted indices in $3 d$ supersymmetric gauge theories, JHEP 08 (2016) 059 [arXiv: 1605.06531] [INSPIRE].

[70] D. Gaiotto, Twisted compactifications of $3 d N=4$ theories and conformal blocks, arXiv: 1611.01528 [INSPIRE].

[71] C. Núñez, I.Y. Park, M. Schvellinger and T.A. Tran, Supergravity duals of gauge theories from $F(4)$ gauged supergravity in six-dimensions, JHEP 04 (2001) 025 [hep-th/0103080] [INSPIRE].

[72] M. Naka, Various wrapped branes from gauged supergravities, hep-th/0206141 [INSPIRE].

[73] Y. Fukuda, T. Kawano and N. Matsumiya, 5D SYM and 2D q-Deformed YM, Nucl. Phys. B 869 (2013) 493 [arXiv:1210.2855] [InSPIRE].

[74] D.M. Hofman and J. Maldacena, Conformal collider physics: Energy and charge correlations, JHEP 05 (2008) 012 [arXiv: 0803.1467] [INSPIRE].

[75] A. Buchel, J. Escobedo, R.C. Myers, M.F. Paulos, A. Sinha and M. Smolkin, Holographic GB gravity in arbitrary dimensions, JHEP 03 (2010) 111 [arXiv:0911.4257] [INSPIRE].

[76] D.M. Hofman, D. Li, D. Meltzer, D. Poland and F. Rejon-Barrera, A Proof of the Conformal Collider Bounds, JHEP 06 (2016) 111 [arXiv:1603.03771] [INSPIRE]. 
[77] Y. Zhou, Information Theoretic Inequalities as Bounds in Superconformal Field Theory, arXiv: 1607.05401 [INSPIRE].

[78] C. Cheung and G.N. Remmen, Positivity of Curvature-Squared Corrections in Gravity, Phys. Rev. Lett. 118 (2017) 051601 [arXiv:1608. 02942] [INSPIRE].

[79] M. Buican, J. Hayling and C. Papageorgakis, Aspects of Superconformal Multiplets in $D>4$, JHEP 11 (2016) 091 [arXiv:1606.00810] [InSPIRE].

[80] C. Cordova, T.T. Dumitrescu and K. Intriligator, Multiplets of Superconformal Symmetry in Diverse Dimensions, arXiv:1612.00809 [INSPIRE].

[81] J.D. Brown and M. Henneaux, Central Charges in the Canonical Realization of Asymptotic Symmetries: An Example from Three-Dimensional Gravity, Commun. Math. Phys. 104 (1986) 207 [INSPIRE].

[82] M. Henningson and K. Skenderis, The Holographic Weyl anomaly, JHEP 07 (1998) 023 [hep-th/9806087] [INSPIRE].

[83] R. Emparan, C.V. Johnson and R.C. Myers, Surface terms as counterterms in the AdS/CFT correspondence, Phys. Rev. D 60 (1999) 104001 [hep-th/9903238] [INSPIRE].

[84] P.K. Townsend and P. van Nieuwenhuizen, Gauged Seven-Dimensional Supergravity, Phys. Lett. B 125 (1983) 41 [INSPIRE].

[85] H. Lü, C.N. Pope, E. Sezgin and K.S. Stelle, Dilatonic p-brane solitons, Phys. Lett. B 371 (1996) 46 [hep-th/9511203] [INSPIRE].

[86] M. Pernici, K. Pilch and P. van Nieuwenhuizen, Gauged Maximally Extended Supergravity in Seven-dimensions, Phys. Lett. B 143 (1984) 103 [InSPIRE].

[87] J.T. Liu and R. Minasian, Black holes and membranes in AdS $S_{7}$, Phys. Lett. B 457 (1999) 39 [hep-th/9903269] [INSPIRE].

[88] M. Pernici and E. Sezgin, Spontaneous Compactification of Seven-dimensional Supergravity Theories, Class. Quant. Grav. 2 (1985) 673 [InSPIRE].

[89] L.J. Romans, The F(4) Gauged Supergravity in Six-dimensions, Nucl. Phys. B 269 (1986) 691 [INSPIRE].

[90] D. Klemm and W.A. Sabra, Supersymmetry of black strings in $D=5$ gauged supergravities, Phys. Rev. D 62 (2000) 024003 [hep-th/0001131] [inSPIRE].

[91] L.J. Romans, Gauged N=4 Supergravities in Five-dimensions and Their Magnetovac Backgrounds, Nucl. Phys. B 267 (1986) 433 [INSPIRE].

[92] M. Günaydin, L.J. Romans and N.P. Warner, Compact and Noncompact Gauged Supergravity Theories in Five-Dimensions, Nucl. Phys. B 272 (1986) 598 [InSPIRE].

[93] H. Nieder and Y. Oz, Supergravity and D-branes wrapping special Lagrangian cycles, JHEP 03 (2001) 008 [hep-th/0011288] [INSPIRE].

[94] O. Aharony, O. Bergman, D.L. Jafferis and J. Maldacena, $N=6$ superconformal Chern-Simons-matter theories, M2-branes and their gravity duals, JHEP 10 (2008) 091 [arXiv: 0806.1218] [INSPIRE].

[95] S.L. Cacciatori and D. Klemm, Supersymmetric AdS 4 black holes and attractors, JHEP 01 (2010) 085 [arXiv: 0911.4926] [INSPIRE]. 
[96] G. Dall'Agata and A. Gnecchi, Flow equations and attractors for black holes in $N=2 \mathrm{U}(1)$ gauged supergravity, JHEP 03 (2011) 037 [arXiv: 1012.3756] [INSPIRE].

[97] K. Hristov and S. Vandoren, Static supersymmetric black holes in $A d S_{4}$ with spherical symmetry, JHEP 04 (2011) 047 [arXiv: 1012.4314] [INSPIRE].

[98] J.P. Gauntlett, N. Kim, S. Pakis and D. Waldram, Membranes wrapped on holomorphic curves, Phys. Rev. D 65 (2002) 026003 [hep-th/0105250] [INSPIRE].

[99] F. Benini, H. Khachatryan and P. Milan, Black hole entropy in massive Type IIA, arXiv:1707.06886 [INSPIRE].

[100] S.M. Hosseini, K. Hristov and A. Passias, Holographic microstate counting for AdS 4 black holes in massive IIA supergravity, JHEP 10 (2017) 190 [arXiv:1707.06884] [INSPIRE].

[101] L.J. Romans, Supersymmetric, cold and lukewarm black holes in cosmological Einstein-Maxwell theory, Nucl. Phys. B 383 (1992) 395 [hep-th/9203018] [INSPIRE].

[102] M.M. Caldarelli and D. Klemm, Supersymmetry of Anti-de Sitter black holes, Nucl. Phys. B 545 (1999) 434 [hep-th/9808097] [INSPIRE].

[103] A. Das, M. Fischler and M. Roček, SuperHiggs Effect in a New Class of Scalar Models and a Model of Super QED, Phys. Rev. D 16 (1977) 3427 [InSPIRE].

[104] M. Cvetič, H. Lü and C.N. Pope, Four-dimensional $N=4$, SO(4) gauged supergravity from $D=11$, Nucl. Phys. B 574 (2000) 761 [hep-th/9910252] [inSPIRE].

[105] J.P. Gauntlett, E. O Colgain and O. Varela, Properties of some conformal field theories with M-theory duals, JHEP 02 (2007) 049 [hep-th/0611219] [INSPIRE].

[106] J.P. Gauntlett and O. Varela, Consistent Kaluza-Klein reductions for general supersymmetric AdS solutions, Phys. Rev. D 76 (2007) 126007 [arXiv:0707.2315] [INSPIRE].

[107] A. Guarino, D.L. Jafferis and O. Varela, String Theory Origin of Dyonic $N=8$ Supergravity and Its Chern-Simons Duals, Phys. Rev. Lett. 115 (2015) 091601 [arXiv: 1504.08009] [INSPIRE].

[108] A. Guarino and O. Varela, Consistent $\mathcal{N}=8$ truncation of massive IIA on $S^{6}$, JHEP 12 (2015) 020 [arXiv: 1509.02526] [inSPIRE].

[109] J.P. Gauntlett and O. Varela, $D=5 \mathrm{SU}(2) \times \mathrm{U}(1)$ Gauged Supergravity from $D=11$ Supergravity, JHEP 02 (2008) 083 [arXiv:0712.3560] [INSPIRE].

[110] H. Lü, C.N. Pope and T.A. Tran, Five-dimensional $N=4, \mathrm{SU}(2) \times \mathrm{U}(1)$ gauged supergravity from type IIB, Phys. Lett. B 475 (2000) 261 [hep-th/9909203] [INSPIRE].

[111] M. Cvetič, H. Lü and C.N. Pope, Gauged six-dimensional supergravity from massive type IIA, Phys. Rev. Lett. 83 (1999) 5226 [hep-th/9906221] [InSPIRE].

[112] J. Jeong, O. Kelekci and E. O Colgain, An alternative IIB embedding of $F(4)$ gauged supergravity, JHEP 05 (2013) 079 [arXiv:1302.2105] [INSPIRE].

[113] E. Malek, Half-maximal supersymmetry from exceptional field theory, Fortsch. Phys. 65 (2017) 1700061 [arXiv:1707.00714] [INSPIRE].

[114] D. Gaiotto, $N=2$ dualities, JHEP 08 (2012) 034 [arXiv:0904.2715] [INSPIRE].

[115] S. Gukov, Trisecting non-Lagrangian theories, JHEP 11 (2017) 178 [arXiv:1707.01515] [INSPIRE]. 
[116] M. Baggio, N. Halmagyi, D.R. Mayerson, D. Robbins and B. Wecht, Higher Derivative Corrections and Central Charges from Wrapped M5-branes, JHEP 12 (2014) 042 [arXiv: 1408.2538] [INSPIRE].

[117] M.T. Anderson, C. Beem, N. Bobev and L. Rastelli, Holographic Uniformization, Commun. Math. Phys. 318 (2013) 429 [arXiv:1109.3724] [INSPIRE].

[118] A. Gadde, S. Gukov and P. Putrov, Fivebranes and 4-manifolds, arXiv:1306.4320 [INSPIRE].

[119] Y. Zhou, Supersymmetric Rényi entropy and Weyl anomalies in six-dimensional $(2,0)$ theories, JHEP 06 (2016) 064 [arXiv: 1512.03008] [INSPIRE].

[120] L. Álvarez-Gaumé and E. Witten, Gravitational Anomalies, Nucl. Phys. B 234 (1984) 269 [INSPIRE]. 